WSRC-TR-2004-00167

Publication Date: May 2004

\title{
Annual Radioactive Waste Tank Inspection Program - 2003(u)
}

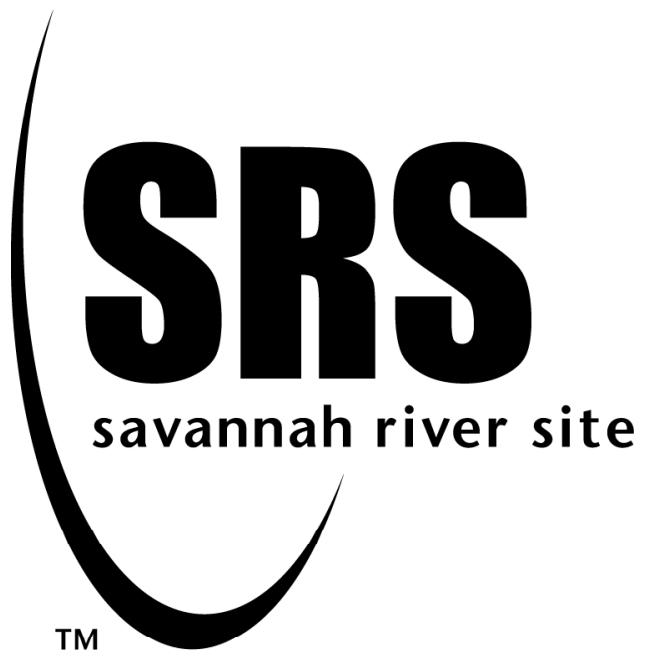

Westinghouse Savannah River Company

Savannah River Site

Aiken, SC 29808

Prepared for the U.S. Department of Energy under Contract No. DE-AC09-96SR18500 
This document was prepared in conjunction with work accomplished under Contract No. DE-AC09-96SR18500 with the U. S. Department of Energy.

\section{DISCLAIMER}

This report was prepared as an account of work sponsored by an agency of the United States Government. Neither the United States Government nor any agency thereof, nor any of their employees, makes any warranty, express or implied, or assumes any legal liability or responsibility for the accuracy, completeness, or usefulness of any information, apparatus, product or process disclosed, or represents that its use would not infringe privately owned rights. Reference herein to any specific commercial product, process or service by trade name, trademark, manufacturer, or otherwise does not necessarily constitute or imply its endorsement, recommendation, or favoring by the United States Government or any agency thereof. The views and opinions of authors expressed herein do not necessarily state or reflect those of the United States Government or any agency thereof.

This report has been reproduced directly from the best available copy.

Available for sale to the public, in paper, from: U.S. Department of Commerce, National Technical Information Service, 5285 Port Royal Road, Springfield, VA 22161, phone: (800) 553-6847, fax: (703) 605-6900

email: orders@ntis.fedworld.gov

online ordering: http://www.ntis.gov/help/index.asp

Available electronically at http://www.osti.gov/bridge

Available for a processing fee to U.S. Department of Energy and its contractors, in paper, from: U.S. Department of Energy, Office of Scientific and Technical Information, P.O. Box 62, Oak Ridge, TN 37831-0062,

phone: (865)576-8401,

fax: (865)576-5728

email: $\underline{\text { reports@ adonis.osti.gov }}$ 
WSRC-TR-2004-00167

Publication Date: May 2004

\section{Annual Radioactive Waste Tank Inspection Program - 2003 ${ }^{(\mathrm{U})}$}

R. S. Waltz, Jr.

W. R. West

Prepared for the U.S. Department of Energy under Contract No. DE-AC09-96SR18500 
This page intentionally left blank. 


\section{Contents}

Acronyms and Abbreviations ....................................... 1

Introduction ..................................................................... 3

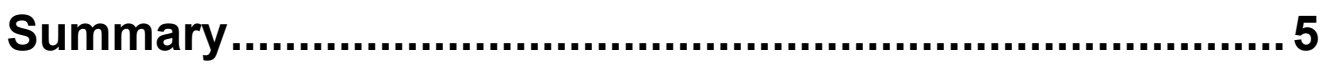

Inspection Program ...................................................... 7

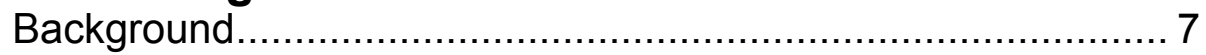

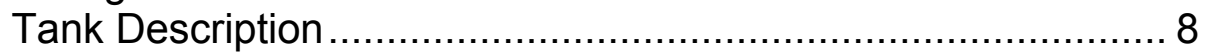

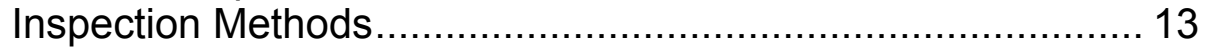

Program Implementation .............................................. 15

Visual Imagery .......................................................... 15

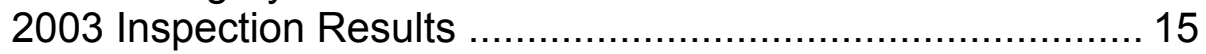

Summary of Inspection Results ........................................ 15

Appendix A-Waste Tanks at SRS...................................23

Appendix B-Summary of 2002 Inspections .................... 25

\section{List of Figures}

1. Type I Waste Storage Tank ............................................. 8

2. Type II Waste Storage Tank .......................................... 9

3. Type IV Waste Storage Tank ........................................ 10

4. Type III Waste Storage Tank ........................................... 11 
This page intentionally left blank. 


\section{Acronyms and Abbreviations}

\begin{tabular}{ll} 
A & Annulus \\
ADMP & Advanced Design Mixer Pump \\
ASME & American Society of Mechanical Engineers \\
BFV & Back Flush Valve \\
CCTV & Closed Circuit Television \\
CCWS & Chromate Cooling Water System \\
COP & Clean Out Port \\
CTS & Concentrate Transfer System \\
CWT & Concentrated Waste Tank \\
DB & Diversion Box \\
DOE-SR & Department of Energy-Savannah River \\
DP & Direct Photography \\
DWPF & Defense Waste Processing Facility \\
ERIP & Encasement Riser Inspection Port \\
ETF & Effluent Treatment Facility \\
EVAP & Evaporator \\
F & Fahrenheit \\
GDL & Gravity Drain Line \\
HELIUM & Helium Leak Test \\
HLLCP & High Liquid Level Conductivity Probe \\
HPFP & High Point Flush Pit \\
I & Interior \\
IAL & Intra-Area Line \\
ISI & In-Service Inspection \\
JB & Junction Box \\
LDB & Leak Detection Box \\
LPPP & Low Point Pump Pit \\
LPS & Leak Probe Sleeve \\
MLDB & Modified Leak Detection Box \\
OD & Outside Diameter \\
PP & Pump Pit \\
psig & pounds per square inch gauge \\
PT & Pump Tank \\
RCP & Reinforced Concrete Pipe \\
SRS & Savannah River Site \\
SSD & Storm Sewer Drain \\
SSMH & Storm Sewer Manhole \\
STE & Shift Technical Engineer \\
SWS & Storm Water Sewer \\
TTJ & Telescopic Transfer Jet \\
TTP & Telescopic Transfer Pump \\
UT & Ultrasonic Nondestructive Examinations \\
VB & Valve Box \\
WAP & Wide-Angle Photography \\
WLE & Waste Line Encasement \\
WSRC & Westinghouse Savannah River Company \\
WT & Waste Transfer Line \\
& \\
& \\
\hline
\end{tabular}


This page intentionally left blank. 
WSRC-TR-2004-00167

Annual Waste Tank

Inspection Program - 2003

Introduction

\section{Introduction}

Aqueous radioactive wastes from Savannah River Site (SRS) separations and vitrification processes are contained in large underground carbon steel tanks. Inspections made during 2003 to evaluate these vessels and other waste

handling facilities along with evaluations based on data from previous inspections are the subject of this report. 
This page intentionally left blank. 
WSRC-TR-2004-00167

Annual Waste Tank

Inspection Program - 2003

\section{Summary}

The 2003 inspection program revealed that the structural integrity and waste confinement capability of the Savannah River Site waste tanks remained unchanged from 2002.

A total of 4249 photographs were made, 1178 visual and video inspections were performed, and 12 helium leak tests were conducted. Ultrasonic testing was performed on 5 High Level Waste Tanks in accordance with approved inspection plans that met the requirements of WSRC-TR-2002-00061, "In-Service Inspection Program for High Level Waste Tanks." 
This page intentionally left blank. 


\section{Inspection Program}

\section{Background}

Alkaline aqueous radioactive wastes produced at the Savannah River Site are received and managed in large underground tanks. The waste came primarily from nuclear fuel reprocessing operations in the separations areas $(\mathrm{F}$ and $\mathrm{H})$ and contains most of the radioactive fission products from SRS operations. In addition, $\mathrm{H}$ tank farm receives recycle waste from the DWPF vitrification process. Some of this waste has been transferred to F Area waste tanks. The waste stored in the tanks is present in three phases: sludge, supernate, and salt formed by supernate evaporation and cooling. The supernate and salt phases consist primarily of $\mathrm{NaNO}_{3}$ and $\mathrm{NaNO}_{2}$. The fission product content is 1 to 20 curies per gallon for the supernate and 1 to 5 curies per gallon for the salt. The sludge consists primarily of $\mathrm{MnO}_{2}$ and $\mathrm{Fe}(\mathrm{OH})_{3}$ with a fission product content up to 410 curies per gallon.

Waste tank leak detection capabilities are essential to meet the primary objective of the SRS radioactive waste management program to manage the waste in such a manner as to minimize the radiation exposure and associated risk to man and his environment over the lifetime of the radionuclides.

The detection of leaked waste is based on two principles: disappearance of material from its proper location and appearance of material in an improper location. At SRS, primary reliance is on the latter because the quantity of the waste detectable in an improper location is much less than that detectable by inventory change in a large tank. Capacity of SRS tanks is 0.75 to 1.3 million gallons. Although rigorous tank inventory surveillance is practiced, primary leak detection methods rely on automatic surveillance of those areas into which the leaked waste is most likely to migrate.

The annulus of each double-wall tank is equipped with at least two single-point conductivity probes for leak detection. These probes are located at the bottom of the annulus and on opposite sides of the tank where possible. The single-wall tanks are built on slabs with a network of leak collection channels that drain to a common sump. Continuous sump level monitoring and frequent sump liquid sampling provide the leak detection. Besides the automatic surveillance, routine direct visual surveys are made in the annular spaces, and nonroutine direct visual surveys are made in primary tanks through opened access risers and/or inspection ports in the roof.

In 1961-62, following leakage of waste into the annuli of Tanks 9, 10,14, and 16, the first remote imaging inspections were made of some tanks using a periscope. Random inspections continued through 1970. A program was initiated in November 1971 to periodically inspect all waste tanks, using remote visual imagery techniques to monitor for corrosion and other degradation, waste leakage, anomalies of any type, and to investigate process or equipment concerns.

Steel thickness measurements have been made periodically of waste tanks using ultrasonic techniques to monitor for general corrosion. An analog-type instrument was used in 1967 and 1969 to measure the thickness of the primary wall of selected double-wall tanks. In 1972, a more precise instrument was put in service. About 24,000 measurements made over a period of 14 years (1972 through 1985) indicated that no general thinning trends of SRS tanks had occurred. Steel thickness measurements were resumed in 1994 using an updated ultrasonic testing (UT) system. The system was updated again in 2002 using improved technology to detect thinning, pitting and cracks.

To date, the only visually observed service-induced corrosion was in Tank 23, a tank with a unique service history. The upper wall interior surfaces show general corrosion with mild pitting. The pitting is broad but shallow. This tank was used to receive contaminated water from 244-H, the Receiving Basin for Off-Site Fuels, and 245-H, the Resin

Regeneration Facility.

Inspections of waste tanks are complicated by factors such as radiation and radioactive contamination, remote operation as far as 40 feet below grade, and insertion of equipment through small (generally 5 to 8-inch-diameter) access openings. Inspection techniques to circumvent these difficulties have been developed, and they yield quality visual images and volumetric measurements. The techniques include photographic systems, closed circuit television 
systems, and ultrasonic systems to measure steel thickness, cracking and pitting.

Waste tank inspection has been important in leak detection. The leaksites in ten of thirteen cracked tanks have been identified by direct visual inspection or by one of the remote inspection techniques. Since the inspection program was initiated in 1971, eight tanks were found to have leaksites that were not recognized before the program was implemented. With one exception, Tank 6 in 2001, the annulus conductivity probes in the double-wall tanks were not activated by these leaks because of the small amount of leakage. The leaked waste evaporated to dryness, sealing the cracks before any leaked waste reached a leak detection probe. However, remote inspections detected the dry deposits of leaked waste in the annuli of these tanks.

The waste tank ISI program is an ongoing program. This report gives results of the 2003 inspections and summarizes significant findings of previous inservice inspections for each waste tank.

\section{Tank Description}

SRS has subsurface storage tanks of four different designs. All of the tanks are constructed of carbon steel and reinforced concrete. They serve as containment vessels for storage and processing of radioactive wastes. Appendix A lists tank location, design type, project number, and construction period. A brief description of the different tank designs is given in the following paragraphs.

\section{Type I Tanks}

The 12 original storage tanks constructed between 1951 and 1953 are designated Type I tanks. Tanks 1 through 8 are in F Area and Tanks 9 through 12 are in $\mathrm{H}$ Area. Each primary tank has a capacity of 750,000 gallons, and is 75 feet in diameter and $241 / 2$ feet high. Figure 1 shows the essential features of Type I tanks, including the primary tank, the secondary pan, and the concrete support structure.

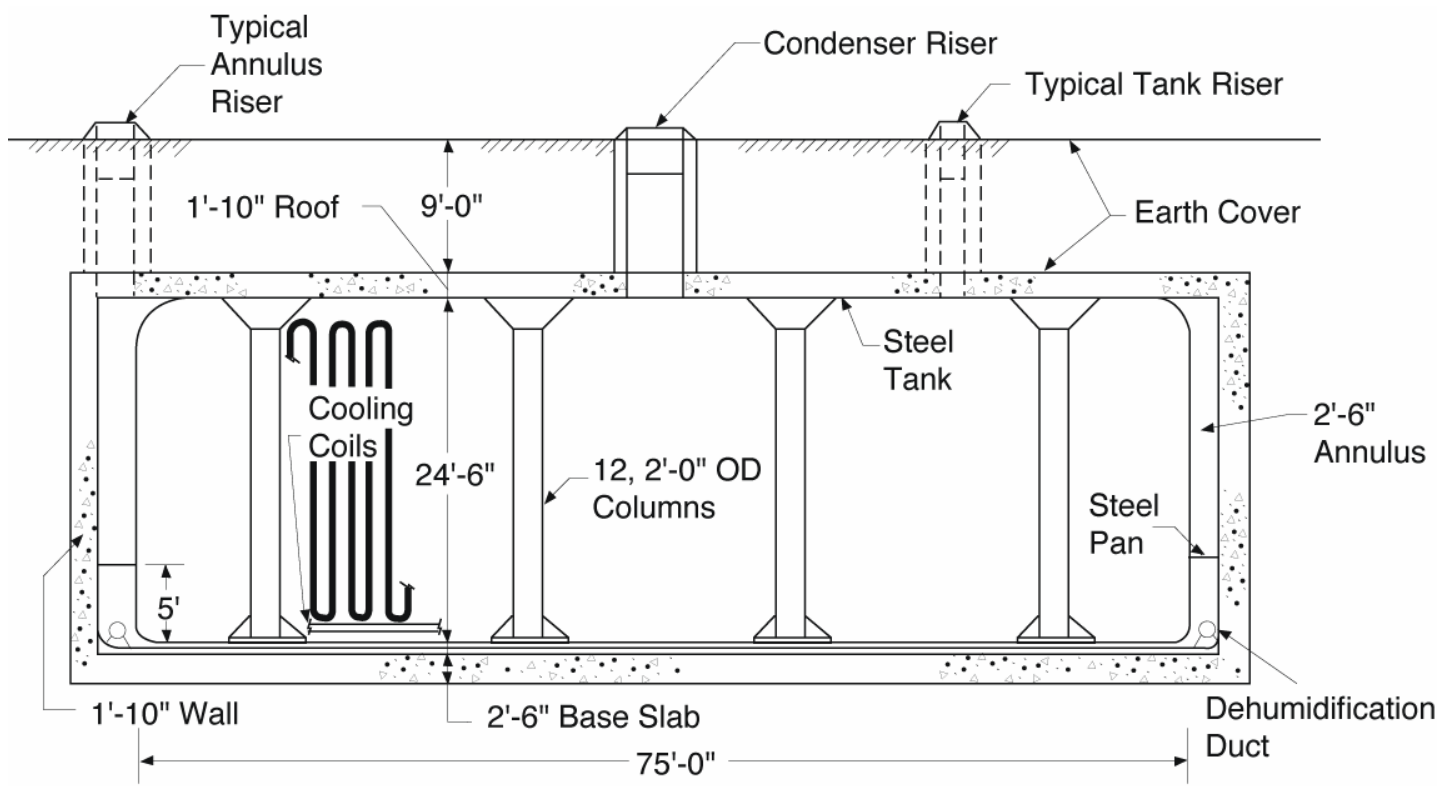

Figure 1. Cooled Waste Storage Tank, Type I (Original 750,000 Gallons) 
The primary container is a closed cylindrical tank with flat top and bottom constructed from 1/2-inchthick steel plates. The top and bottom are joined to the cylindrical sidewall by curved knuckle plates. The primary tank is set within a circular pan of 1/2inch-thick steel plates. The annulus pan is 5 feet deep and 5 feet larger in diameter than the primary tank, thus forming an annular space $21 / 2$ feet wide. The tank and pan are set on a 30-inch-thick base slab and are enclosed by a cylindrical 22-inch-thick reinforced concrete wall and a flat concrete roof, also 22 inches thick. There are twelve 2-foot-diameter concrete columns within the primary tank to support the roof. Each column has a flared capital and is encased in 1/2-inch-thick steel plate.

A 9-foot layer of earth was placed over the tanks for radiation shielding. Cooling for each Type I tank is provided by 36 parallel (water pipe) cooling coils.

A dehumidification duct in the annulus of each tank is routed from the tank top to the bottom of the annulus where it encircles all but 8 feet of tank. The duct has distribution outlets and its cross-sectional area decreases as the distance from the air supply increases. Access to the tank interior is provided at eight locations, and to the annular space at four locations, through riser pipes. Each of the 12 riser pipes is capped at the top with a concrete plug. Each plug is provided with two 5 -inch-diameter ports equipped with removable plugs unless equipment has been installed to support waste removal or tank closure activities. The center plug port may provide access through three 4 to 8 inch diameter ports. Some of these ports provide access for inspections.

All welds in the pan and primary tank were radiographically inspected, defects were corrected, and the welds were rechecked radiographically. The welds in the flat bottoms of both the pan and the tank were vacuum-tested for leaks. Additionally, both vessels were hydrostatically tested. The water was maintained at full height in the tank for 24 hours before inspection for leaks was made. Cooling water piping was hydrostatically tested at $300 \mathrm{psig}$ and then leak-tested with 100 psig air pressure in the piping.

\section{Type II Tanks}

Tanks 13 through 16, constructed in H Area in 1955 and 1956, are designated Type II tanks. Figure 2 is a cross section of this type. Each primary tank has a capacity of 1,030,000 gallons and is 85 feet in diameter and 27 feet high.

The primary container for Type II tanks consists of two concentric steel cylinders assembled with a flat bottom and a flat top into a form somewhat like a doughnut. The top and bottom are joined to the outer cylinder by rings of curved knuckle plates. The inner cylinder is flared at the top to accommodate the roof support column. This cylinder is joined to the flat steel top with a continuous butt weld and to a base fastened to the bottom with a continuous T-weld. Steel thicknesses are:

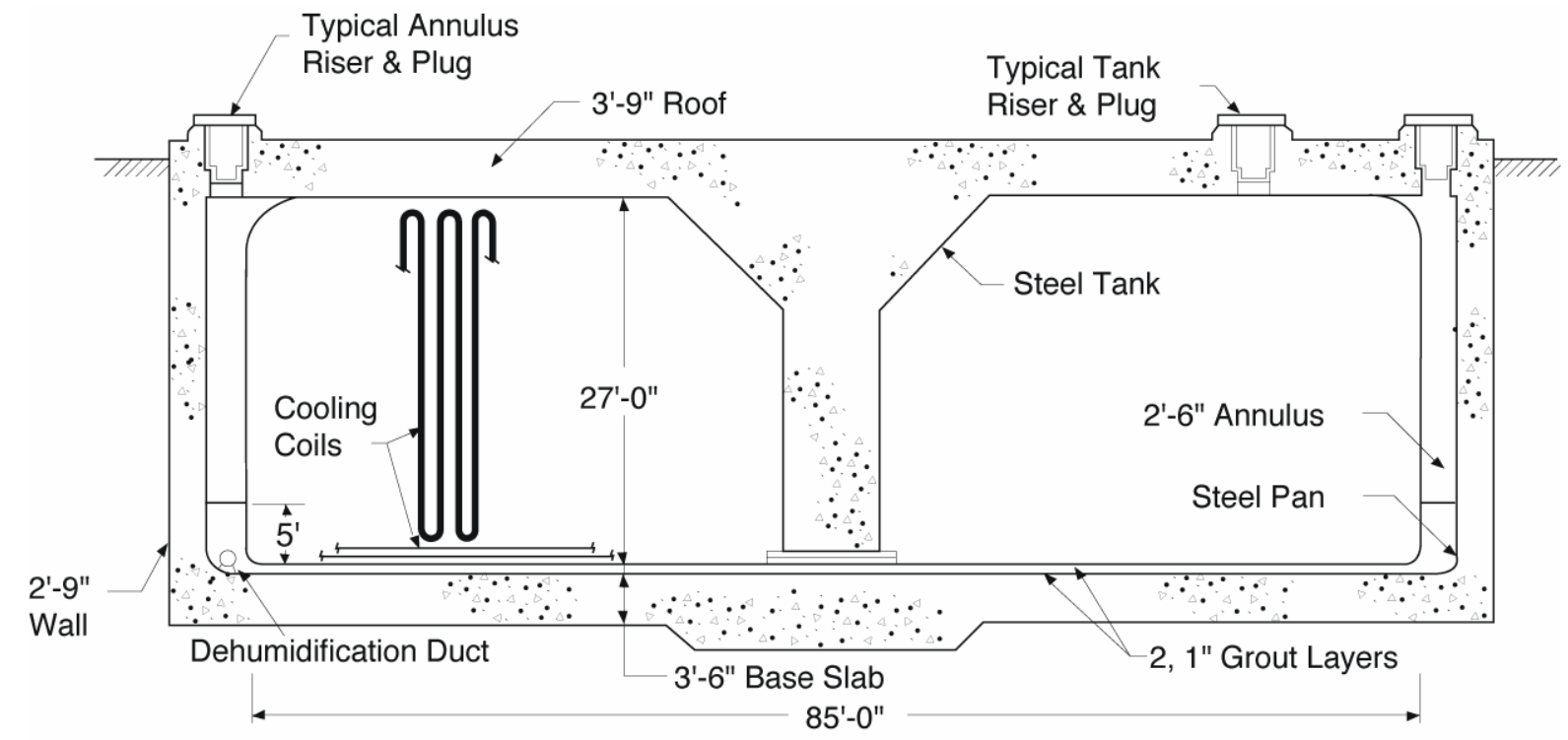

Figure 2. Cooled Waste Storage Tank, Type II (Original 1,030,000 Gallons) 
Plate

Top and bottom

Top knuckle

Wall

Bottom knuckle

The primary tank is set on a 1-inch sand bed within a circular pan of 1/2-inch thick steel plate, 5 feet deep and 5 feet larger in diameter than the primary tank, thus forming an annular space $21 / 2$ feet wide. The tank and pan assembly is surrounded by a cylindrical reinforced concrete enclosure with a 33-inch-thick wall and a flat concrete roof that is 45 inches thick. The tank and pan assembly and the surrounding wall are set on a foundation slab that is 42 inches thick. The roof is supported by both the wall and a central concrete column that fits within the inner cylinder of the vessel. The 45 -inch-thick concrete roof provides radiation shielding; therefore, no earth overburden is required. Cooling for each Type II tank is provided by 44 parallel (water pipe) cooling coils. Access to the tank interior is provided at eight locations, and to the annular space at four locations, through riser pipes. Each of the 12 riser pipes is capped at the top with a concrete plug. Each plug is provided with two 5-inch-diameter ports equipped with removable plugs. The ports provide access for inspection. In addition to the four annulus risers, other access openings (10 to 14 additional openings per tank) have been drilled into the annulus of each of these tanks to permit inspection of seventy-three to ninety-six percent of the exterior walls of the primary vessels.

A dehumidification duct in the annulus of each tank is routed from the tank top to the bottom of the annulus where it encircles all but 8 feet of the tank. The duct has distribution outlets, and its crosssectional area decreases as the distance from the air supply increases.

All welds in the primary tanks were radiographically inspected, defects were corrected, and the welds were rechecked radiographically. However, the annulus pans were not inspected radiographically. The welds in the flat bottoms of these pans and the primary tanks were vacuum-tested for leaks, and the primary and secondary vessels were hydrostatically tested. Cooling water piping was hydrostatically tested at 300 psig and then leak-tested, with 100 psig air pressure in the piping.

\section{Type IV Tanks}

Tanks 17 through 24 are single-wall-uncooled tanks. These tanks were designed for storage of waste that does not require auxiliary cooling. Tanks 17 through 20 were constructed in F Area in 1956 and Tanks 21 through 24 were constructed in H Area in 1960. Each tank has a capacity of 1,300,000 gallons and is 85 feet in diameter and 34 feet high (Figure 3).

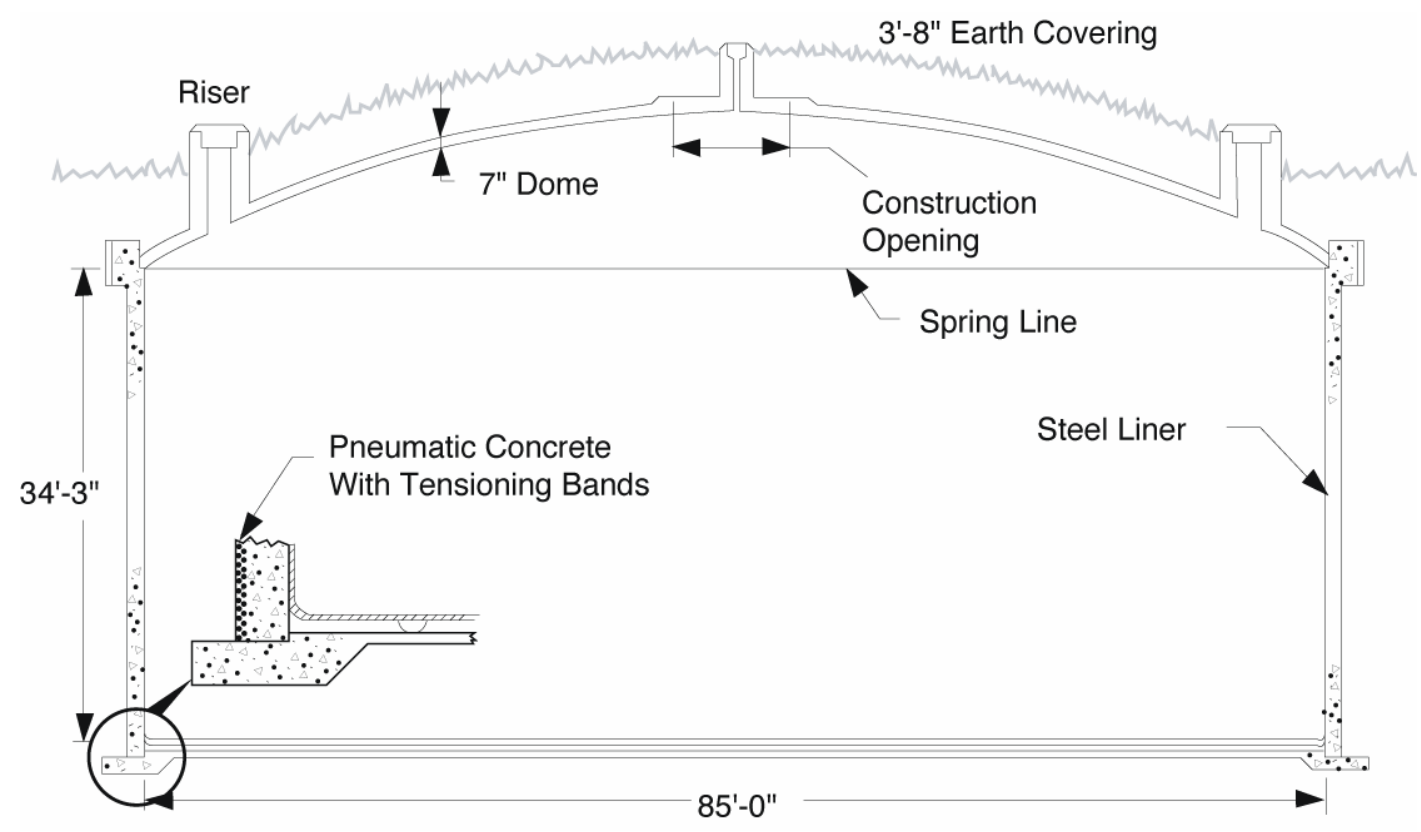

Figure 3. Uncooled Waste Storage Tank, Type IV (Prestressed Concrete Walls, 1,300,000 Gallons) 
WSRC-TR-2004-00167

Annual Waste Tank

Each Type IV tank is basically a steel-lined, prestressed-concrete tank in the form of a vertical cylinder with a domed roof. Carbon steel plates, 3/8 inch thick, were used to form the cylindrical sides and flat bottom portion of the steel liners. The knuckle plates at the junction of the bottom and the sidewall are 7/16 inch thick. Concrete was built up around the steel vessel by the "shotcrete" technique.

Radiation shielding of the Type IV tanks in F Area was accomplished by applying at least 32 inches of earth over each of the 7-inch-thick concrete domes. H-Area tanks were shielded similarly, except that the earth cover was at least 44 inches thick to accommodate a somewhat higher radiation level from the waste.

Access to the interior of the tank is provided at six locations through riser pipes. Each riser pipe is capped at the top with a concrete plug. Some of these risers provide access for inspection.

All welds in the steel liners were radiographically inspected. All of the welded tank-bottom seams and the upper seams of the knuckle rings were vacuum leak-tested. Prior to the back-filling operation, each tank was hydrostatically tested by filling with water to the normal fill line. The tank was allowed to remain filled until it was to be placed in use for waste storage.

\section{Type III Tanks}

The most recently constructed tanks are designated as Type III tanks (Figure 4). Twenty-seven tanks were built between 1966 and 1981. Tanks 25 through 28, 33 and 34, and 44 through 47 are located in F Area. Tanks 29 through 32, 35 through 43 and 48 through 51 are located in H Area.

The Type III tank design was developed after an investigation into the causes of the leaks from the primary vessel of the Type I and Type II tanks. The study concluded that the leak-producing mechanism was nitrate-induced, stress-corrosion cracking at sites in or near the weld seams, and that stress relieving after fabrication should eliminate the cracking. For the Type III tanks, means were provided for heating each finished tank to relieve the stresses generated during fabrication. In addition, some stress patterns were avoided, or minimized, by mounting the roof supporting column on the foundation pad rather than on the bottom of the primary tank (as in Types I and II), and by providing an annular clearance around the roof supporting column. Each primary tank holds $1,300,000$ gallons and is 85 feet in diameter and 33 feet high.

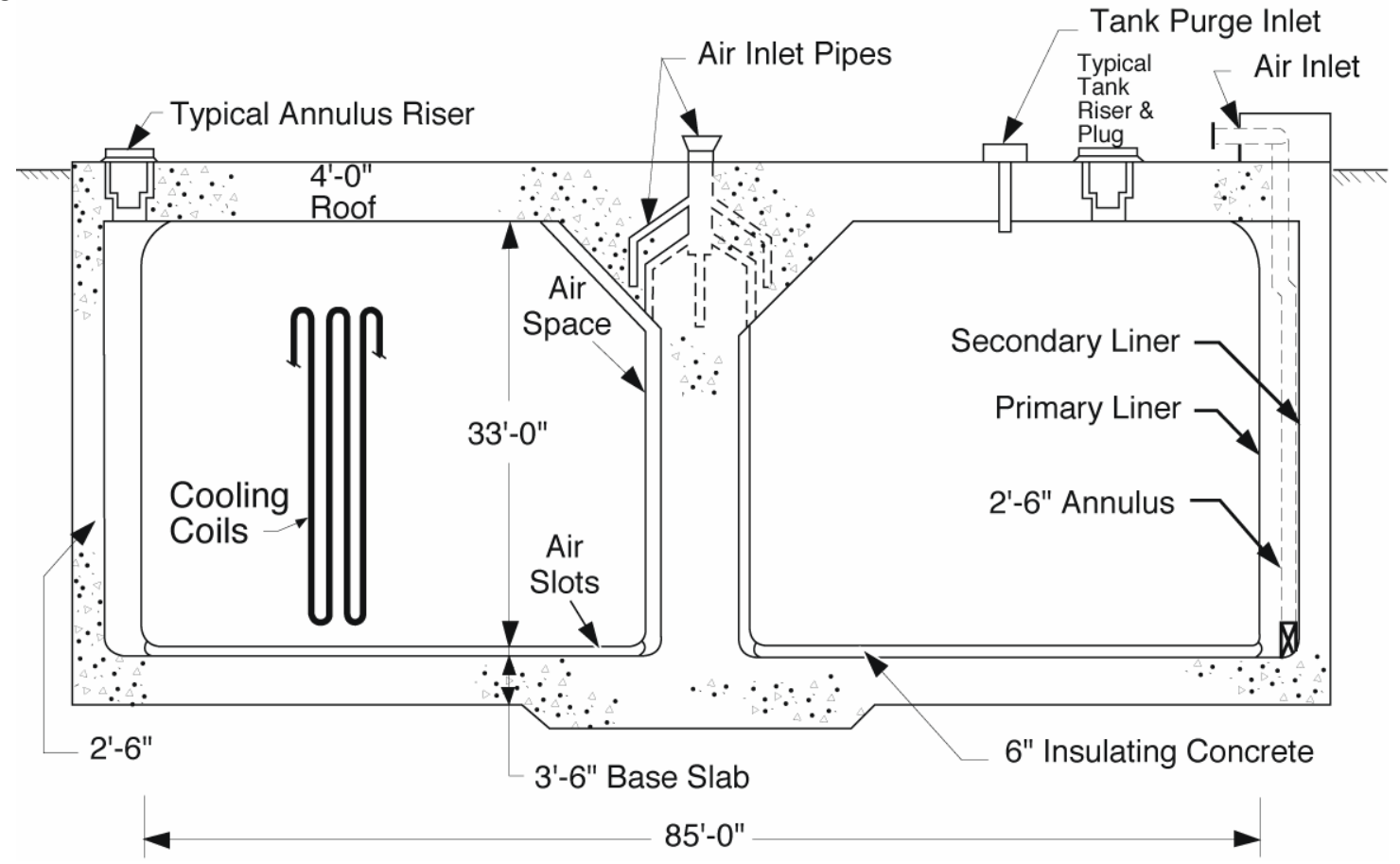

Figure 4. Cooled Waste Storage Tank, Type III (Stress Relieved Primary Liner, 1,300,000 Gallons) 
Type III tanks are similar to the doughnut-like design of Type II tanks. Each primary vessel is made of two concentric cylinders joined to washer-shaped top and bottom plates by curved knuckle plates. Steel thicknesses are:

\section{Plate}

Thickness, inch

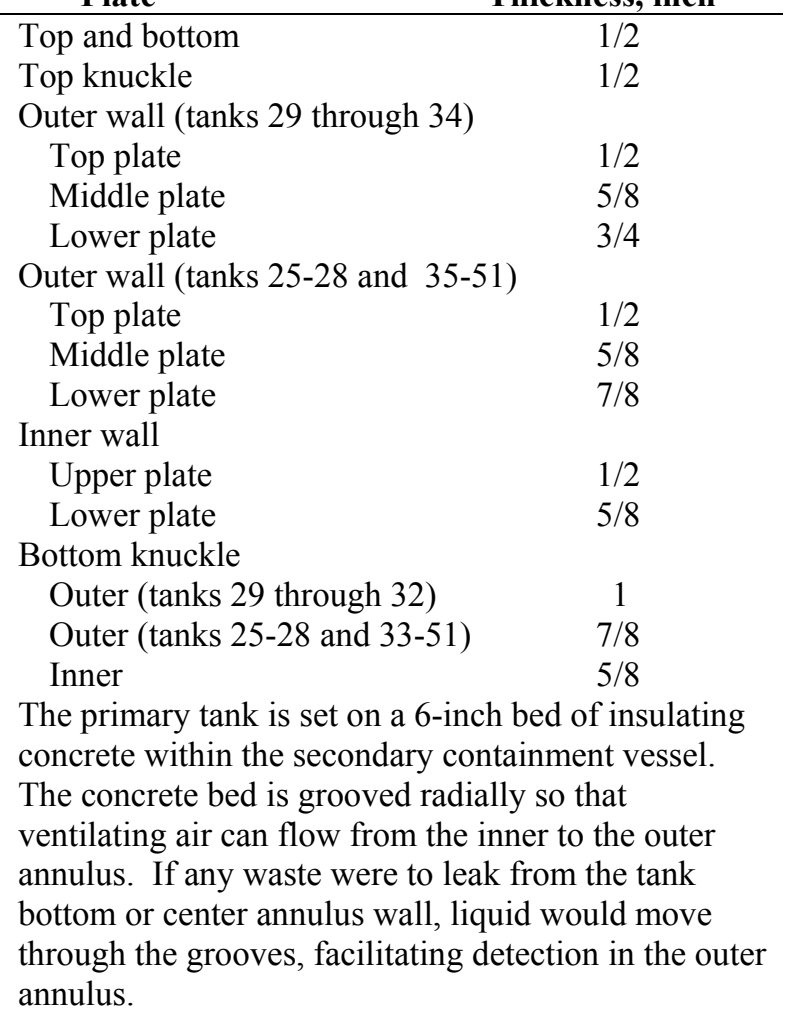

The secondary vessel is 5 feet larger in diameter than the tank, thus providing an outer annulus $21 / 2$ feet wide. The secondary vessel is made of $3 / 8$-inch-thick steel throughout. Its sidewalls rise to the full height of the primary tank. The nested two-vessel assembly is surrounded by a cylindrical reinforced concrete enclosure with a 30-inch-thick wall. The enclosure has a 48-inch-thick flat reinforced concrete roof that is supported by the concrete wall and a central column that fits within the inner cylinder of the vessel. The 48 -inch-thick concrete provides radiation shielding; hence, no earth overburden is required.

Cooling for the Type III tanks is provided by either deployable (water pipe) cooling coil bundles installed through risers in the tank top, or 23 parallel (water pipe) cooling coils distributed throughout the tank.

A dehumidification duct in the annulus of each tank is routed from the tank top to the bottom of the annulus where it encircles the tank. The duct has distribution outlets and its cross-sectional area decreases as distance from the air supply increases. In these tanks, additional airflow is directed through the inner annulus, passing beneath the primary tank through radial grooves in the concrete base slab, and is exhausted into the outer annulus.

Tanks 29 through 34 were placed in service prior to 1976. These tanks were constructed with annulus riser pipes at four locations providing inspection access through 5-inch-diameter ports. All other Type III tanks were placed in service after 1976 and have annulus riser pipes at 18 locations that are 8-inches in diameter. These ports are equidistant around the tank and provide for inspection of all of the exterior wall of the primary vessel. In 1982, fourteen to sixteen additional 8-inch diameter ports per tank were drilled in the tops of Tanks 29 through 34 to provide adequate access ports for inspection of all of the exterior wall of their primary vessels. All Type III tanks have interior riser pipes at various locations that provide inspection access through ports with diameters ranging from 4 to 8 inches. All inspection access ports are equipped with removable plugs.

All butt welds on the primary tanks were radiographically inspected, except welds on the horizontal roof surface. On the secondary vessels of Tanks 29 through 34, all butt welds joining bottom plates, knuckle plates, and the lowest courses of center-column and outer-wall plates, were radiographically inspected. On all other Type III tanks, all plate welds in the secondary tanks were radiographically inspected. All defects were corrected and the welds were rechecked radiographically.

The Quality Assurance Program included inspection of all radiographs by two independent groups of certified weld inspectors, and all radiographs were permanently stored for future reference. All spots on the inside or outside of the primary tanks and the inside of the secondary tanks, where clips or lugs were removed and where other excisions were made, were examined by magnetic particle or liquid penetrant techniques, and any defects were repaired.

All butt welds on the secondary tanks were vacuum leak-tested. All welds in the bottom assemblies of the primary tanks, including knuckle rings and lowest course welds, were vacuum leak-tested before each bottom assembly was lowered into final position, and then tested a second time after the stress-relieving operation. A full hydrostatic test, the filling of each primary tank to a depth of 32 feet and allowing it to stand 48 hours, was conducted after stress relieving. 
WSRC-TR-2004-00167

Annual Waste Tank

No leaks were found by the hydrostatic tests. All circumferential welds in the pipe loops of the deployable cooling coil bundles below the $1 / 2$-inchthick plate at the base of the riser plug were radiographed. The assembled cooler piping was tested hydrostatically to 500 psig and halide leaktested at $300 \mathrm{psig}$. Welds in the distributed cooling coils were radiographed and similarly leak-tested.

The primary tank was stress-relieved in place after all high temperature work (other than roof attachments) had been completed. Full stress relief, at $1100^{\circ} \mathrm{F}$, was accomplished in accordance with the general requirements of the ASME Boiler and Pressure Vessel code.

\section{Inspection Methods}

Techniques have been developed for remote examination and evaluation of the waste tanks and waste tank ancillaries. For visual imaging, direct photography systems developed at SRS were the primary method used. Closed circuit television systems were also used where direct photography was not possible or where these systems provided a more comprehensive examination. Only the direct photography systems will be described since the video systems are similar to systems used widely in industry.
Wide-angle direct photography was used for general inspections of double-wall tank annuli and the primary vessels of both double-wall tanks and singlewall tanks. This technique used a camera that surveys a large area in a single photograph. The camera used for wide-angle photography was a Contax G1 camera body, with a Zeiss Hologon $16 \mathrm{~mm} \mathrm{f} / 8$ fixed aperture lens. This lens is distortion free with a field of view of approximately 100 degrees. A bank of four electronic flash units was synchronized with these cameras to provide illumination. This camera is not shielded since residence time in a radiation field is minimal.

Another direct photography technique was used for detailed inspections. The camera is shielded to reduce the degrading effect of ionizing radiation on the photographic film. The camera's residence time in a radiation field for this technique is longer than the wide-angle direct photographic technique (i.e., a few minutes versus a few seconds); hence, shielding is required. The camera used was the Contax G1 camera with a Zeiss Hologon $16 \mathrm{~mm} \mathrm{f} / 8$ lens, the same as used for the wide-angle direct photography. Illumination is provided by a single electronic flash unit. 
WSRC-TR-2004-00167

Annual Waste Tank

This page intentionally left blank. 


\section{Program Implementation}

\section{Visual Imagery}

The 2003 inspection program used two visual imagery techniques: photography and closed circuit television. The primary inspection methods were direct photography techniques; e.g., making a series of photographs providing detailed views of the tank and wide-angle photography for obtaining overviews of large areas. Closed circuit television systems were generally used to further investigate conditions found during scheduled inspections and to document conditions and troubleshoot process problems in tanks and ancillaries.

The inspection program objective to continuously evaluate the waste tanks was satisfied in 2003 by photographic and videotape documentation.

Inspections were made through all accessible annulus risers of the double-wall tanks and at least one inspection was made in the interior of each singlewall tank.

For Tanks 1 through 12, inspections are limited to no more than $25 \%$ of the exterior of the primary vessel wall and the annular space due to limited annulus access. These tanks are continuously monitored for leakage by instrumentation installed in their annuli.

Additionally, for those tanks that have known leaksites in the primary vessel, the supernate phase has been removed, minimized, or the level lowered below the level of known leaksites. Inspections revealing no changes on these tanks are denoted as "Tank condition had not changed."

\section{Inspection Results}

The 2003 inspection program was successfully completed. The annuli of all double-wall tanks were inspected via all accessible risers and the interiors of single-wall tanks remaining in service were inspected. Other inspections of waste tanks and ancillaries were performed as required by operating conditions and equipment performance.

Per the In Service Inspection Program Plan, ultrasonic nondestructive examinations were performed for Tanks 26, 30, 31, 32 and 34. A full scope examination was performed at Tanks 26 and
32. This included four 8.5 inch wide vertical strips for the entire accessible height, $10 \%$ of a horizontal weld and a vertical weld on the top plate. The tank was examined for wall thinning, crack detection, flaw sizing and pitting. Reduced augmented scope examinations were performed on Tanks 30, 31 and 34. This consisted of examining one 8.5 inch wide vertical strip for the entire accessible height for wall thinning, crack detection, flaw sizing and pitting. Examinations of the secondary vessel wall were performed on accessible plates for thinning and pitting beneath one riser in both full scope and augmented inspections. A small area of reportable thickness ( 0.334 inches) was detected on the $4^{\text {th }}$ plate of the secondary vessel in Tank 26 beneath the P-05 riser. No other areas of reportable cracking, thinning, or pitting were detected. Details of these inspections are included in Appendix B.

Rainwater continued to leak into the annulus of some tanks. Inleakage was evidenced primarily by surface stains, and occasionally by calciferous deposits, changed configuration in leaked waste in the annulus, and mild surface corrosion where annulus ventilation was inadequate to maintain a dry annulus.

Except as noted above, the conditions of the tanks remained essentially unchanged from the conditions reported in 2002. Details and results for inspections of the tanks and ancillaries performed in 2003 are listed in Appendix B.

\section{Summary of Inspection Results}

The following is a brief description of tank conditions as revealed by inspections and examinations made through 2003.

\section{Tank 1}

Tank 1 was placed in service in 1954. A small amount of dry waste was observed on the annulus floor in 1969. Subsequent inspections have revealed no additional leakage. Tank 1 was removed from active service in December 1985. Inspection of the exterior wall of the primary vessel is limited to $25 \%$ using existing inspection techniques through the four 
risers that provide access to the annulus. Visual examinations of the observable portion of the tank wall have not revealed the location of the leak(s). Inspection photographs of the steel surface of the tank and the annulus have shown no significant surface corrosion or other anomalies. Ultrasonic measurements made in $1978,1979,1981,1983$, and 1985 showed that no detectable thinning of the tank wall had occurred.

\section{Tank 2}

Tank 2 was placed in service in 1955 . Tank 2 was removed from active service in November 1984. Visual examinations of the observable portion (25\%) of the exterior of the primary vessel wall and the annulus have shown no leakage, significant surface corrosion or other anomalies. Ultrasonic measurements made in 1967, 1972, 1973, 1977, 1981, and 1985 showed no detectable thinning of the tank wall.

\section{Tank 3}

Tank 3 was placed in service in 1956. Tank 3 was removed from active service in November 1984. Visual examinations of the observable portion $(25 \%)$ of the exterior of the primary vessel wall and the annulus have shown no leakage, significant surface corrosion or other anomalies. Ultrasonic measurements made in 1973, 1977, 1981, and 1985 showed no detectable thinning of the tank wall.

\section{Tank 4}

Tank 4 was placed in service in 1961. Tank 4 was removed from active service in December 1979. Visual examinations of the observable portion (25\%) of the exterior of the primary vessel wall and the annulus have shown no leakage, significant surface corrosion or other anomalies. Ultrasonic measurements made in 1973, 1977, 1981, and 1985 showed no detectable thinning of the tank wall.

\section{Tank 5}

Tank 5 was placed in service in 1959. Tank 5 was removed from active service in November 1990. Visual examinations of the observable portions $(25 \%)$ of the exterior of the primary vessel wall and the annulus through calendar year 2000 had shown no leakage, significant surface corrosion, or other anomalies. The tank was returned to active service in 2001 to support tank closure activities. Several months after liquid was added to the tank, a magnetically mounted wall crawler with a video camera attached was deployed which enabled $75 \%$ of the primary vessel wall to be inspected. These inspections revealed 15 leaksites. Less than 5 gallons of waste reached the annulus floor. Waste was removed from the tank to a level below the lowest known leaksite. Ultrasonic measurements made in 1973, 1977, 1981, and 1985 showed no detectable thinning of the tank wall.

\section{Tank 6}

Tank 6 was placed in service in 1964. Tank 6 was removed from active service in October 1990. Visual examinations of the observable portions $(25 \%)$ of the exterior of the primary vessel wall and the annulus through calendar year 2000 had shown no leakage, significant surface corrosion, or other anomalies. The tank was returned to active service in 2000 to support tank closure activities. The first indication of leakage from the tank was in January 2001 when an annulus conductivity probe alarm was received. Liquid was observed on the annulus floor; however, no leaksites could be located from the four risers used to inspect the tank. In February 2001, a magnetically mounted wall crawler with a video camera attached enabled $73 \%$ of the primary vessel wall to be examined. These inspections identified 6 leaksites. Approximately 92 gallons of waste reached the annulus floor. Waste was removed from the tank to a level below the lowest known leaksite. Ultrasonic measurements made in 1974, 1977, 1978, 1979, 1981, and 1985 showed no detectable thinning of the tank wall.

\section{Tank 7}

Tank 7 was placed in service in 1954. Tank 7 was removed from active service in November 1989. Visual examinations of the observable portion (25\%) of the exterior of the primary vessel wall and the annulus have shown no leakage, significant surface corrosion or other anomalies. Ultrasonic measurements made in 1974, 1979, 1981, 1983, and 1985 showed no detectable thinning of the tank wall. Tank 7 was returned to active service in 2002 to support waste removal activities.

\section{Tank 8}

Tank 8 was placed in service in 1956. Tank 8 was removed from active service in September 1992. Tank 8 was returned to active service in 2001 to support waste removal activities.

Visual examinations of the observable portion (25\%) of the exterior of the primary vessel wall and the annulus have shown no leakage, significant surface 
WSRC-TR-2004-00167

Annual Waste Tank

Inspection Program - 2003

Program Implementation

corrosion or other anomalies. A magnetically mounted wall crawler with a video camera attached enabled inspection of $59 \%$ of the primary vessel wall and annulus. No leakage, significant surface corrosion, or other anomalies were observed. Ultrasonic measurements made in 1973, 1977, 1981, and 1985 showed no detectable thinning of the tank wall.

\section{Tank 9}

Tank 9 was placed in service in 1955. Tank 9 was removed from active service in July 1991. Leakage from the tank primary vessel into the annulus pan may have occurred as early as 1955 when the "necklace" alarm, a conductivity leak detection device, shorted out permanently. Leakage was not certain until liquid waste was observed in the annulus pan in 1957. Currently, the annulus pan contains 8 to 10 inches of dry leaked waste. Visual examinations of the observable portion (13\%) of the exterior of the primary vessel wall have shown three leaksites high on the tank wall; 269, 271, and 276 inches above the tank bottom. None of these leaksites is the source of the leaked waste in the annulus pan. The waste leaked at these sites was only enough to form localized small nodules. The leak(s) that are the source of the waste in the annulus pan have not been observed. Inspections have shown no significant surface corrosion, and the ultrasonic measurements made in 1979 and 1983 showed no detectable thinning of the tank wall.

\section{Tank 10}

Tank 10 was placed in service in 1955. Tank 10 was removed from active service in August 1989. The first indication that Tank 10 had leaked was in 1959 when dry waste was discovered in the annulus pan during a visual inspection. Currently, the annulus pan contains about 2 - 3 inches of dry leaked waste. Visual examinations of the observable portion (19\%) of the exterior of the primary vessel wall have not shown the source of the leaked waste or any other leaksite(s). Inspections have shown no significant surface corrosion, and the ultrasonic measurements made in 1979 and 1983 showed no detectable thinning of the tank wall.

\section{Tank 11}

Tank 11 was placed in service in 1955. Tank 11 was removed from active service in July 1989.

Inspections performed in 1974 revealed two leaksites. The leaksites are 189 and 235 inches above the tank bottom. Visual examinations of the observable portions (25\%) of the exterior of the primary vessel wall have shown no significant surface corrosion, and ultrasonic measurements made in 1973, 1977, 1981, and 1985 showed no detectable thinning of the tank wall.

\section{Tank 12}

Tank 12 was placed in service in 1956 . Tank 12 was removed from active service in July 1990. Inspections in 1974 revealed two leaksites. The leaksites are 93 and 105 inches above the tank bottom. Visual examinations of the observable portions $(25 \%)$ of the exterior of the primary vessel wall have shown no significant surface corrosion, and ultrasonic measurements made in 1972, 1973, 1977, 1981, 1983, and 1985 showed no detectable thinning of the tank wall.

\section{Tank 13}

Tank 13 was placed in service in 1956. Tank 13 was removed from active service in May 1991. Ninety percent of the exterior of the primary vessel wall is observable via the 13 risers that provide access to the annulus. Inspections in 1977 revealed a leaksite 279 inches above the tank bottom. In 1980, another leaksite was discovered 269 inches above the tank bottom. Visual examinations have shown no significant surface corrosion, and ultrasonic measurements made in 1974, 1979, 1985 and 2000* showed no detectable thinning of the tank wall. (* this corrects an omission from the 2001 report and an error in the 2002 report)

\section{Tank 14}

Tank 14 was placed in service in 1957. Tank 14 was removed from active service in December 1991. The first indication that tank 14 had leaked was in 1959 when dry leaked waste was observed in the annulus pan. Currently, the annulus pan contains 12 to 13 inches of dry leaked waste. Eighty-nine percent of the exterior of the primary vessel wall is observable via the 18 risers that provide access to the annulus. Inspections have located 33 leaksites, and it is estimated that there are about 50 leaksites in this tank. All of the observed leaksites are near the bottom circumferential weld that is 2.5 feet above the tank bottom, except for one leaksite that was observed approximately 248 inches above the tank bottom. Visual examinations have shown no significant surface corrosion, and ultrasonic measurements made in 1979 and 1983 showed no detectable thinning of the tank wall. 


\section{Tank 15}

Tank 15 was placed in service in 1960 . Tank 15 was removed from active service in November 1989. Inspections in 1972 below one of the four risers providing access to the annulus revealed two leaksites near the bottom circumferential weld about 2.5 feet above the tank bottom. Twelve additional risers were installed, increasing the observable portion of the primary vessel wall from $25 \%$ to $96 \%$. Inspections in 1973, via the additional risers, revealed eleven other leaksites.

Later, inspections revealed three other sites where cracks penetrated the steel wall, one was observed in 1994 and two were observed in 1997. Inspections in 2000 revealed two additional leaksites near the bottom circumferential weld. A total of 18 leaksites have been identified.

Visual examinations have shown mild corrosion of the steel surfaces in the tank annulus. Ultrasonic measurements made in 1972, 1977, 1980, 1984 and 2002 showed no reportable thinning of the tank wall. Using new equipment capable of detecting cracks and pitting, four partial through-wall crack indications and one complete through-wall crack were identified during UT mapping in 2002 that were previously unknown.

\section{Tank 16}

Tank 16 was placed in service in 1959. Tank 16 was removed from active service in February 1979. Liquid waste was detected in the annulus pan in 1959. Seventy-three percent of the exterior wall of the primary vessel is observable via the sixteen risers that provide access to the annulus. Inspections in 1961 and 1962, through 13 risers, revealed about 175 leaksites in the tank wall. In October 1961 and March 1962, two 5 3/4-inch-diameter samples were cut from the top horizontal circumferential weld of the tank wall about 40 feet apart. Metallurgical examination indicated the cause of the cracks was nitrate-induced stress corrosion. Extensive inspection performed since 1972 indicated that the primary vessel wall has 300 to 350 leaksites. In $1978,70 \%$ of the leaked waste in the annulus pan was removed, leaving an insoluble heel containing approximately 30,000 curies Cs- 137 . Waste removal from the interior of the primary vessel was completed in 1980, and the tank status changed to "out of service". Visual examinations have shown no significant surface corrosion. No ultrasonic steel thickness measurements of the tank were made because of the number of leaksites and the presence of leaked waste deposits on the primary vessel exterior.

\section{Tank 17}

Tank 17 was placed in service in 1961. Visual examinations of the steel liner revealed no evidence of failure, significant surface corrosion or other anomalies. Tank 17 was removed from service and closed December 15, 1997. Inspections have been discontinued.

\section{Tank 18}

Tank 18 was placed in service in 1959. Visual examinations of the steel liner have shown no evidence of failure, significant surface corrosion or other anomalies. Ultrasonic measurements made in 1977, 1980, and 1983 showed no detectable thinning of the liner bottom. Activities to remove all waste from the tank began in 2001. Tank 18 currently has a waste "heel" of approximately 8425 gallons and is awaiting final closure. The tank was removed from service in 2003.

\section{Tank 19}

Tank 19 was placed in service in 1961 and emptied in 1981. Visual examinations of the steel liner have revealed two failures; i.e. sites where inleakage had occurred. The failures are in the wall of the steel liner at heights of 317 inches and 330 inches. Inspection records photographically document that these leaksites existed before 1994. However, inspections made from the interior of this single-wall (visual inspection of the exterior is not possible) had to track changes in artifacts at the sites by periodic observation to judge that inleakage had occurred. Ultrasonic measurements made in 1982 and 1985 showed no detectable thinning of the liner bottom. Activities to remove all waste from the tank began in 2000. Tank 19 currently has a waste "heel" of approximately 15,576 gallons and is awaiting final closure. The tank was removed from service in 2003.

\section{Tank 20}

Tank 20 was placed in service in 1960 . Visual examinations of the steel liner revealed four failure sites. In 1983, leaksites were observed in the wall of the steel liner at heights of 22, 24.5, and 26.5 feet. In 1990 , a leaksite was confirmed in the liner wall at a height of 26.25 feet. This site had been suspect since 1984. This is a single-wall tank with no annulus. The leaksites in the steel liner were detected by inspections made from the tank interior, since 
WSRC-TR-2004-00167

Annual Waste Tank

Inspection Program - 2003

Program Implementation

inspection of the exterior was not possible. Artifacts observed on the interior wall indicated water had leaked through the steel liner into the tank. It is possible that a small quantity of waste may have leaked from the steel liner. However, groundwater monitoring has given no indication that waste escaped the encasement. Tank 20 was removed from service and closed July 31, 1997. Inspections have been discontinued.

\section{Tank 21}

Tank 21 was placed in service in 1961 . Visual examinations of the steel liner have shown no evidence of failure, significant surface corrosion or other anomalies. Ultrasonic measurements made in 1973, 1977, 1980, and 1983 showed no detectable thinning of the liner bottom.

\section{Tank 22}

Tank 22 was placed in service in 1965 . Visual examinations of the steel liner have shown no evidence of failure, significant surface corrosion or other anomalies. Water was discovered leaking through the concrete roof in 1994. This water intrusion was attributed to the presence of perched water on top of the dome (PEC-SMS-96-0084). The condition will continue to be monitored. Ultrasonic measurements made in 1974, 1977, 1980, and 1983 showed no detectable thinning of the liner bottom.

\section{Tank 23}

Tank 23 was placed in service in 1964 . Visual examinations of the steel liner have revealed corrosion but no evidence of failure. Ultrasonic measurements made in 1973, 1977, 1980, and 1983 showed no detectable thinning of the liner bottom. Examinations of the steel liner have shown rust and tubercles on the surface of the upper portion. This tank served as a receiver tank for inhibited contaminated water from Buildings 244-H, the Receiving Basin for Off-Site Fuels, and 245-H, the Resin Regeneration Facility. The tank was filled to less than $50 \%$ capacity to maintain the remaining space for emergency use. This mode of operation exposed only the lower half of the tank to the inhibited contents and exposed the upper half of the tank to a warm humid atmosphere. In 1984, rust and tubercles were cleaned from two small areas, exposing the steel surface. The cleaned liner surface was generally corroded with mild pitting. The pits were broad and shallow. In 1999, cracked or crushed concrete was noted in the tank dome, spanning about fifteen feet immediately above the tank wall. The structural integrity of the dome was evaluated (T-CLC-H-00444 and T-CLC-H-00447). The dome meets AC1318-95 code requirements and will continue to be monitored.

\section{Tank 24}

Tank 24 was placed in service in 1963. Visual examinations of the steel liner have shown no evidence of failure, significant surface corrosion or other anomalies. Ultrasonic measurements made in 1984 showed no detectable thinning of the liner.

\section{Tank 25}

Tank 25 was placed in service in 1980 . Visual examinations of $100 \%$ of the exterior of the primary vessel wall and the annulus have shown no leakage, significant surface corrosion or other anomalies. Ultrasonic thickness measurements made in 1979 and 1983 showed no detectable thinning of the tank wall.

\section{Tank 26}

Tank 26 was placed in service in 1980. Visual examinations of $100 \%$ of the exterior of the primary vessel wall and the annulus have shown no leakage, significant surface corrosion or other anomalies. Ultrasonic thickness measurements made in 1979 and 1983 showed no detectable thinning of the tank wall. UT measurements made in 2003 showed no reportable thinning, pitting or stress corrosion cracking.

\section{Tank 27}

Tank 27 was placed in service in 1980. Visual examinations of $100 \%$ of the exterior of the primary vessel wall and the annulus have shown no leakage, significant surface corrosion or other anomalies. Ultrasonic thickness measurements made in 1979 and 1983 showed no detectable thinning of the tank wall.

\section{Tank 28}

Tank 28 was placed in service in 1980. Visual examinations of $100 \%$ of the exterior of the primary vessel wall and the annulus have shown no leakage, significant surface corrosion or other anomalies. Ultrasonic thickness measurements made in 1979 and 1983 showed no detectable thinning of the tank wall.

\section{Tank 29}

Tank 29 was placed in service in 1971. Visual examinations of $100 \%$ of the exterior of the primary vessel wall and the annulus have shown no leakage, 
significant surface corrosion or other anomalies. Ultrasonic thickness measurements made in 1973 and 1974 showed no detectable thinning of the tank wall.

\section{Tank 30}

Tank 30 was placed in service in 1974 . Visual examinations of $100 \%$ of the exterior of the primary vessel wall and the annulus have shown no leakage, significant surface corrosion or other anomalies. Ultrasonic thickness measurements made in 1975 showed no detectable thinning of the tank wall. UT measurements made in 2003 showed no reportable thinning, pitting or stress corrosion cracking.

\section{Tank 31}

Tank 31 was placed in service in 1972. Visual examinations of $100 \%$ of the exterior of the primary vessel wall and the annulus have shown no leakage, significant surface corrosion or other anomalies. Ultrasonic thickness measurements made in 2003 showed no reportable thinning, pitting or stress corrosion cracking of the tank wall.

\section{Tank 32}

Tank 32 was placed in service in 1971. Visual examinations of $100 \%$ of the exterior of the primary vessel wall and the annulus have shown no leakage, significant surface corrosion or other anomalies. Ultrasonic thickness measurements made in 2003 showed no reportable thinning, pitting or stress corrosion cracking of the tank wall.

\section{Tank 33}

Tank 33 was placed in service in 1969. Visual examinations of $100 \%$ of the exterior of the primary vessel wall and the annulus have shown no leakage, significant surface corrosion or other anomalies.

\section{Tank 34}

Tank 34 was placed in service in 1972. Visual examinations of $100 \%$ of the exterior of the primary vessel wall and the annulus have shown no leakage, significant surface corrosion or other anomalies. Ultrasonic thickness measurements made in 2003 showed no reportable thinning, pitting or stress corrosion cracking of the tank wall.

\section{Tank 35}

Tank 35 was placed in service in $1977 . \quad$ Visual examinations of $100 \%$ of the exterior of the primary vessel wall and the annulus have shown no leakage, significant surface corrosion or other anomalies. Ultrasonic thickness measurements made in 1977 , 1981, and 1985 showed no detectable thinning of the tank wall.

\section{Tank 36}

Tank 36 was placed in service in 1977. Visual examinations of $100 \%$ of the exterior of the primary vessel wall and the annulus have shown no leakage, significant surface corrosion or other anomalies. Ultrasonic thickness measurements made in 1977 , 1981, and 1985 showed no detectable thinning of the tank wall.

\section{Tank 37}

Tank 37 was placed in service in 1978. Visual examinations of $100 \%$ of the exterior of the primary vessel wall and the annulus have shown no leakage, significant surface corrosion or other anomalies. Ultrasonic thickness measurements made in 1977 , 1981, and 1985 showed no detectable thinning of the tank wall.

\section{Tank 38}

Tank 38 was placed in service in 1981. Visual examinations of $100 \%$ of the exterior of the primary vessel wall and the annulus have shown no leakage, significant surface corrosion or other anomalies. Ultrasonic thickness measurements made in 1980 , 1981, and 1984 showed no detectable thinning of the tank wall.

\section{Tank 39}

Tank 39 was placed in service in 1982. Visual examinations of $100 \%$ of the exterior of the primary vessel wall and the annulus have shown no leakage, significant surface corrosion or other anomalies. Ultrasonic thickness measurements made in 1980, 1981, 1984, and 1985 showed no detectable thinning of the tank wall.

\section{Tank 40}

Tank 40 was placed in service in 1986 . Visual examinations of $100 \%$ of the exterior of the primary vessel wall and the annulus have shown no leakage, significant surface corrosion or other anomalies. Ultrasonic thickness measurements were made in 1980, 1981, and 1984. Thickness mapping was performed in 1996 using the P-scan System to provide reference measurements for the future. No reportable service-induced pitting or wall thinning was detected. 
WSRC-TR-2004-00167

Annual Waste Tank

Inspection Program - 2003

Program Implementation

\section{Tank 41}

Tank 41 was placed in service in 1982. Visual examinations of $100 \%$ of the exterior of the primary vessel wall and the annulus have shown no leakage, significant surface corrosion or other anomalies. Ultrasonic thickness measurements made in 1980, 1981, and 1984 showed no detectable thinning of the tank wall.

\section{Tank 42}

Tank 42 was placed in service in 1982. Visual examinations of $100 \%$ of the exterior of the primary vessel wall and the annulus have shown no leakage, significant surface corrosion or other anomalies. Ultrasonic thickness measurements were made in 1980, 1981, 1984, 1985 and 1990. Thickness mapping was performed in 1995 and 1996 using the P-scan System to provide reference measurements for the future. No reportable service-induced pitting or wall thinning was detected.

\section{Tank 43}

Tank 43 was placed in service in 1982. Visual examinations of $100 \%$ of the exterior of the primary vessel wall and the annulus have shown no leakage, significant surface corrosion or other anomalies. Ultrasonic thickness measurements made in 1980, 1981, 1984, and 1985 showed no detectable thinning of the tank wall.

\section{Tank 44}

Tank 44 was placed in service in 1982. Visual examinations of $100 \%$ of the exterior of the primary vessel wall and the annulus have shown no leakage, significant surface corrosion or other anomalies. Ultrasonic thickness measurements made in 1980, 1981, and 1984 showed no detectable thinning of the tank wall.

\section{Tank 45}

Tank 45 was placed in service in 1982. Visual examinations of $100 \%$ of the exterior of the primary vessel wall and the annulus have shown no leakage, significant surface corrosion or other anomalies. Ultrasonic thickness measurements made in 1980, 1981, and 1984 showed no detectable thinning of the tank wall.

\section{Tank 46}

Tank 46 was placed in service as an emergency spare tank in 1980. It was placed in waste storage service in 1994 when it began receiving concentrate from the $2 \mathrm{~F}$ evaporator. Visual examinations of $100 \%$ of the exterior of the primary vessel wall and the annulus have shown no significant surface corrosion or other anomalies. Ultrasonic thickness measurements made in 1980, 1981, and 1984 showed no detectable thinning of the tank wall.

\section{Tank 47}

Tank 47 was placed in service in 1980. Visual examinations of $100 \%$ of the exterior of the primary vessel wall and the annulus have shown no leakage, significant surface corrosion or other anomalies. Ultrasonic thickness measurements made in 1980, 1981, and 1984 showed no detectable thinning of the tank wall.

\section{Tank 48}

Tank 48 was placed in service in 1983. Visual examinations of $100 \%$ of the exterior of the primary vessel wall and the annulus have shown no leakage, significant surface corrosion or other anomalies. Ultrasonic thickness measurements were made in 1982 prior to placing the tank in service. Thickness mapping was performed in 1994, 1995, 1996, and 1997 using the P-scan System to provide reference measurements for the future. No reportable serviceinduced pitting or wall thinning was detected.

\section{Tank 49}

Tank 49 was placed in service in 1983. Visual examinations of $100 \%$ of the exterior of the primary vessel wall and the annulus have shown no leakage, significant surface corrosion or other anomalies. Ultrasonic thickness measurements were made in 1982 prior to placing the tank in service. Thickness mapping was performed in 1995 using the P-scan System to provide reference measurements for the future. No reportable service-induced pitting or wall thinning was detected.

\section{Tank 50}

Tank 50 was placed in service in 1983. Visual examinations of $100 \%$ of the exterior of the primary vessel wall and the annulus have shown no leakage, significant surface corrosion or other anomalies. Ultrasonic thickness measurements were made in 1982 prior to placing the tank in service. Thickness mapping was performed in 1994 and 1995 using the P-scan System to provide reference measurements for the future. No reportable service-induced pitting or wall thinning was detected. 


\section{Tank 51}

Tank 51 was placed in service in 1986. Visual examinations of $100 \%$ of the exterior of the primary vessel wall and the annulus have shown no leakage, significant surface corrosion or other anomalies.
Ultrasonic thickness measurements were made in 1982 prior to placing the tank in service. Thickness mapping was performed in 1996 and 1997 using the P-scan System to provide reference measurements for the future. No reportable service-induced pitting or wall was detected. 
WSRC-TR-2004-00167

Annual Waste Tank

Inspection Program - 2003

Appendix A-Waste Tanks at SRS

\section{Appendix A-Waste Tanks at SRS}

SRS Waste Tank Specifications

\begin{tabular}{lccccc} 
Number & Location & Type & $\begin{array}{c}\text { Project } \\
\text { Number }\end{array}$ & $\begin{array}{c}\text { Construction } \\
\text { Period }\end{array}$ & $\begin{array}{c}\text { Type of } \\
\text { Construction* }\end{array}$ \\
\hline $1-8$ & F & I & 8980 & $1951-1953$ & Double wall-cooled \\
$9-12$ & H & I & 8980 & $1951-1953$ & Double wall-cooled \\
$13-16$ & H & II & 8980 & $1955-1956$ & Double wall-cooled \\
$17-20$ & F & IV & 981031 & 1958 & Single wall-uncooled \\
$21-24$ & H & IV & 981089 & 1962 & Single wall-uncooled \\
$25-28$ & F & IIIA & 951493 & $1975-1978$ & Double wall-cooled \\
$29-32$ & H & III & 981232 & $1967-1970$ & Double wall-cooled \\
$33-34$ & F & III & 950974 & $1969-1972$ & Double wall-cooled \\
$35-37$ & H & IIIA & 951463 & $1974-1977$ & Double wall-cooled \\
$38-43$ & H & IIIA & 951618 & $1976-1980$ & Double wall-cooled \\
& & & $(76-8-\mathrm{A})$ & & \\
$44-47$ & F & IIIA & 951747 & $1977-1980$ & Double wall-cooled \\
$48-51$ & H & IIIA & 951828 & $1978-1981$ & Double wall-cooled \\
& & & $(78-18-b)$ & &
\end{tabular}

* Tanks 32 and 35 have removable, roof-supported cooling coils. Tanks 30, 33, and 34 have bottom-supported deployable cooling coils. Tanks 29 and 31 have some deployable and some close-packed cooling assemblies, all bottom supported. All other cooled tanks have permanently installed cooling coils, roof-supported in Type I and II and bottom-supported in Type III tanks. 
WSRC-TR-2004-00167

Annual Waste Tank

Appendix A-Waste Tanks at SRS

Inspection Program - 2003

This page intentionally left blank. 


\section{Appendix B-Summary of 2003 Inspections}

\begin{tabular}{|c|c|c|c|c|c|c|c|c|}
\hline \multirow{2}{*}{$\begin{array}{c}\text { AREA } \\
F\end{array}$} & \multirow{2}{*}{$\begin{array}{c}\begin{array}{c}\text { TANK OR } \\
\text { ANCILLARY }\end{array} \\
01\end{array}$} & \multicolumn{2}{|c|}{$\frac{\text { ACCESS OPENING }}{\text { (A OR I) }}$} & \multirow{2}{*}{$\begin{array}{c}\text { DATE } \\
01 / 30 / 03\end{array}$} & \multicolumn{3}{|c|}{$\frac{\text { INSPECTION METHOD }}{\text { IDENTIFICATION NUMBER }}$} & \multirow{2}{*}{$\begin{array}{l}\text { REMARKS } \\
\text { The magnetically mounted } \\
\text { thermocouple was deployed per } \\
\text { Structural Integrity Data Sheet } \\
\text { T-DS-G-00010. }\end{array}$} \\
\hline & & East & (A) & & CCTV & I & 911 & \\
\hline $\mathrm{F}$ & 01 & East & (A) & 07/19/03 & CCTV & I & 926 & $\begin{array}{l}\text { The conductivity probe was } \\
\text { properly deployed per setpoint } \\
\text { document J-JX-G-0001. }\end{array}$ \\
\hline $\mathrm{F}$ & 01 & East & (A) & 07/19/03 & CCTV & l & 926 & $\begin{array}{l}\text { The magnetically mounted } \\
\text { thermocouple was improperly } \\
\text { positioned. It was detached from } \\
\text { the tank wall. }\end{array}$ \\
\hline $\mathrm{F}$ & 01 & East & (A) & 07/19/03 & DP & l & P03147:01-27 & Tank condition had not changed. \\
\hline $\mathrm{F}$ & 01 & East & (A) & $07 / 24 / 03$ & CCTV & l & 926 & $\begin{array}{l}\text { The magnetically mounted } \\
\text { thermocouple was deployed per } \\
\text { Structural Integrity Data Sheet } \\
\text { T-DS-G-00010. }\end{array}$ \\
\hline $\mathrm{F}$ & 01 & North & (A) & 07/17/03 & WAP & l & P03152:01 & Tank condition had not changed. \\
\hline $\mathrm{F}$ & 01 & South & (A) & 07/19/03 & DP & l & P03145:01-23 & Tank condition had not changed. \\
\hline $\mathrm{F}$ & 01 & West & (A) & 07/19/03 & CCTV & l & 926 & $\begin{array}{l}\text { The conductivity probe was } \\
\text { properly deployed per setpoint } \\
\text { document J-JX-G-0001. }\end{array}$ \\
\hline $\mathrm{F}$ & 01 & West & (A) & 07/19/03 & DP & I & P03146:01-22 & Tank condition had not changed. \\
\hline $\mathrm{F}$ & 02 & East & (A) & $06 / 28 / 03$ & WAP & I & P03131:01 & Tank condition was normal. \\
\hline $\mathrm{F}$ & 02 & East & $(\mathrm{A})$ & 06/29/03 & CCTV & I & 926 & $\begin{array}{l}\text { The magnetically mounted } \\
\text { thermocouple was deployed per } \\
\text { Structural Integrity Data Sheet } \\
\text { T-DS-G-00010. }\end{array}$ \\
\hline $\mathrm{F}$ & 02 & North & (A) & 06/28/03 & WAP & I & P03131:02 & Tank condition was normal. \\
\hline $\mathrm{F}$ & 02 & North & $(\mathrm{A})$ & 06/29/03 & CCTV & I & 926 & $\begin{array}{l}\text { The conductivity probe was } \\
\text { properly deployed per setpoint } \\
\text { document J-JX-G-0001. }\end{array}$ \\
\hline $\mathrm{F}$ & 02 & South & (A) & $06 / 28 / 03$ & DP & I & P03124:01-22 & Tank condition was normal. \\
\hline $\mathrm{F}$ & 02 & South & (A) & 06/29/03 & CCTV & I & 926 & $\begin{array}{l}\text { The conductivity probe was } \\
\text { properly deployed per setpoint } \\
\text { document J-JX-G-0001. }\end{array}$ \\
\hline $\mathrm{F}$ & 02 & West & (A) & 06/28/03 & WAP & I & P03131:03 & Tank condition was normal. \\
\hline
\end{tabular}




\section{TANK OR \\ AREA}

$\mathrm{F}$

02

$\frac{\frac{\text { ACCESS OPENING }}{\text { (A OR I) }}}{01 \quad(\mathrm{I})} \quad \frac{\text { DATE }}{07 / 22 / 03}$

INSPECTION METHOD IDENTIFICATION NUMBER

CCTV / $\quad 991$

\section{REMARKS}

Inspection documented conditions of the spray chamber, sleeve and area beneath the riser. No unusual conditions were observed. Liquid was observed beneath the riser. Salt formations were observed on the cooling coils and on the surface of the waste. Salt was attached to the coils suspended above the liquid spanning several coils.

CCTV / 1004

CCTV / 1004

09/03/03

02 (I)

$09 / 03 / 03$

\section{East (A)}

06/28/03

06/29/03

North (A)
03

03

03

03

03

03
North (A)

South (A)

South

(A)

West

West (A)

$06 / 29 / 03$

06/29/03

$06 / 28 / 03$

06/29/03

06/28/03

WAP

CCTV

DP

/ P03127:01-22

WAP

CCTV

926

F

08/01/03
CCTV / 991
Inspection verified position of sample rod dropped during removal. The rod was upright beneath riser 1 .

Inspection documented position of sample tool rod that was dropped during removal. The rod was standing upright beneath riser 1 .

Tank condition was normal.

The conductivity probe was properly deployed per setpoint document J-JX-G-0001.

Tank condition was normal.

Tank condition was normal. properly deployed per setpoint document J-JX-G-0001.

Tank condition was normal.

The magnetically mounted thermocouple was deployed per Structural Integrity Data Sheet T-DS-G-00010.

Inspection documented the area beneath the riser. No obstructions were observed. Water dripping and deposits on cooling coil \#8 indicate that a leak exists at a hanger below the riser.
The conductivity probe was 


\section{TANK OR \\ AREA}

F

03

$\frac{\text { ACCESS OPENING }}{(A \text { OR I) }}$

DATE

01 (I)

$11 / 11 / 03$

CCTV / 1040

CCTV / 1040

INSPECTION METHOD

IDENTIFICATION NUMBER

CCTV I 945

05 (I)

$04 / 27 / 03$

CCTV

I

945

CCTV

982

07/02/03

COTV

I

07/30/03

CCTV I

991

$07 \quad$ (I)

$11 / 11 / 03$

03
$\mathrm{F}$

11/11/03

\section{CCTV I}

\section{REMARKS}

Inspection was made to document waste configuration and tank conditions. The surface of the waste was irregular saltcake. One cooling coil was observed leaking, and several coils had damp areas indicating leaksites exist at these locations. Salt formations were observed on the cooling coils and tank wall.

Inspection documented the conditions of the tank contents and transfer jet. The jet suction leg was below the surface of the waste. Salt formations were observed on the cooling coils. The waste surface was salt with a pool of liquid beneath the riser and the reel tape in riser 4 . No unusual conditions were observed.

Inspection was performed to document jet piping connections inside the riser and tank conditions beneath the riser. The jet connections were flanges, and the waste surface was salt with a small pool of liquid observed beneath the riser. No unusual conditions were observed.

Inspection of the steam side of the transfer jet was performed. No unusual conditions were observed.

Inspection revealed the waste level in the caisson to be the same as the tank. No unusual conditions were observed.

Inspection was made to document waste configuration and tank conditions. Several cooling coils were damp at the hanger indicating leaksites may exist there. No broken hangers were identified. The waste was salt and appeared to be uniform across the tank. However, there were some depressions observed which indicated voids beneath the salt layer. Abandoned steel tapes were observed beneath the riser. 


\begin{tabular}{|c|c|c|c|c|}
\hline AREA & $\begin{array}{l}\text { TANK OR } \\
\text { ANCILLARY }\end{array}$ & $\frac{\text { ACCESS OPENING }}{(A \text { OR I })}$ & DATE & $\frac{\text { INSPECTION METHOD }}{\text { IDENTIFICATION NUMBER }}$ \\
\hline $\mathrm{F}$ & 03 & Center & 04/27/03 & CCTV \\
\hline
\end{tabular}

\section{REMARKS}

Inspection documented the condition of the tank contents. The waste surface was salt with salt formations on the cooling coils. No unusual conditions were observed.

Inspection of the dip tube legs revealed no unusual conditions.

Inspection documented the waste surface. Liquid was observed throughout the tank with a crystallized layer of salt forming on top. Salt formations were observed on the cooling coils.

CCTV

1038

Inspection was made to document waste configuration and tank conditions. The surface of the waste was irregular saltcake with salt attached to the roof supported cooling coils suspended above the waste level spanning several coils. Chromate deposits were observed on several vertical and horizontal coils indicating leaksites exist at these locations.

Inspection was made to document waste configuration and tank conditions. The surface of the waste was irregular saltcake with salt attached to the roof supported cooling coils suspended above the waste level spanning several coils. No liquid was observed.
Inspection was made to document waste configuration and tank conditions. The surface of the waste was irregular salt cake. Three cooling coils were observed leaking, and several coils were damp at the hanger indicating leaksites may exist at these locations. Numerous chromate deposits were also observed. No broken hangers were identified.

Tank condition was normal.

The magnetically mounted thermocouple was deployed per Structural Integrity Data Sheet T-DS-G-00010. 


\begin{tabular}{|c|c|c|c|c|c|c|c|c|}
\hline \multirow{2}{*}{$\frac{\text { AREA }}{F}$} & \multirow{2}{*}{$\begin{array}{c}\begin{array}{c}\text { TANK OR } \\
\text { ANCILLARY }\end{array} \\
04\end{array}$} & \multicolumn{2}{|c|}{$\frac{\text { ACCESS OPENING }}{(A \text { OR I) }}$} & \multirow{2}{*}{$\frac{\text { DATE }}{06 / 29 / 03}$} & \multicolumn{3}{|c|}{$\begin{array}{l}\text { INSPECTION METHOD } \\
\text { IDENTIFICATION NUMBER }\end{array}$} & \multirow{2}{*}{$\begin{array}{l}\qquad \text { REMARKS } \\
\qquad \begin{array}{l}\text { The conductivity probe was } \\
\text { properly deployed per setpoint } \\
\text { document J-JX-G-0001. }\end{array}\end{array}$} \\
\hline & & North & (A) & & CCTV & l & 926 & \\
\hline $\mathrm{F}$ & 04 & South & (A) & $06 / 29 / 03$ & CCTV & l & 926 & $\begin{array}{l}\text { The conductivity probe was } \\
\text { properly deployed per setpoint } \\
\text { document J-JX-G-0001. }\end{array}$ \\
\hline $\mathrm{F}$ & 04 & South & (A) & $06 / 29 / 03$ & $\mathrm{DP}$ & l & P03130:01-22 & Tank condition was normal. \\
\hline $\mathrm{F}$ & 04 & West & (A) & 06/28/03 & WAP & l & P03128:01 & Tank condition was normal. \\
\hline $\mathrm{F}$ & 04 & 01 & (I) & $10 / 20 / 03$ & CCTV & l & 1029 & $\begin{array}{l}\text { Inspection revealed one cooling } \\
\text { coil pair positioned within the } \\
\text { diameter of the riser opening, } \\
\text { which could obstruct installation of } \\
\text { a slurry pump. }\end{array}$ \\
\hline $\mathrm{F}$ & 04 & 04 & (I) & $10 / 21 / 03$ & CCTV & l & 1029 & $\begin{array}{l}\text { Inspection was made to document } \\
\text { equipment configuration beneath } \\
\text { all visible tank risers. No unusual } \\
\text { conditions were observed. }\end{array}$ \\
\hline $\mathrm{F}$ & 04 & 08 & (I) & $10 / 20 / 03$ & CCTV & l & 1029 & $\begin{array}{l}\text { Inspection revealed no } \\
\text { obstructions beneath the riser } \\
\text { which would obstruct installation of } \\
\text { a slurry pump. }\end{array}$ \\
\hline $\mathrm{F}$ & 04 & Center & (I) & $10 / 21 / 03$ & CCTV & l & 1029 & $\begin{array}{l}\text { Inspection was made to document } \\
\text { equipment configuration beneath } \\
\text { all visible tank risers. No unusual } \\
\text { conditions were observed. }\end{array}$ \\
\hline $\mathrm{F}$ & 05 & East & (A) & $07 / 02 / 03$ & DP & l & P03133:01-22 & Tank condition was normal. \\
\hline $\mathrm{F}$ & 05 & North & (A) & $07 / 02 / 03$ & DP & l & P03134:01-22 & Tank condition was normal. \\
\hline $\mathrm{F}$ & 05 & North & (A) & 07/06/03 & CCTV & l & 926 & $\begin{array}{l}\text { The conductivity probe was } \\
\text { properly deployed per setpoint } \\
\text { document J-JX-G-0001. }\end{array}$ \\
\hline $\mathrm{F}$ & 05 & South & $(\mathrm{A})$ & $07 / 17 / 03$ & WAP & l & P03151:01 & Tank condition had not changed. \\
\hline $\mathrm{F}$ & 05 & South & (A) & $07 / 18 / 03$ & CCTV & I & 926 & $\begin{array}{l}\text { The conductivity probe was } \\
\text { properly deployed per setpoint } \\
\text { document J-JX-G-0001. }\end{array}$ \\
\hline $\mathrm{F}$ & 05 & West & (A) & 07/06/03 & CCTV & I & 926 & $\begin{array}{l}\text { The magnetically mounted } \\
\text { thermocouple was deployed per } \\
\text { Structural Integrity Data Sheet } \\
\text { T-DS-G-00010. }\end{array}$ \\
\hline $\mathrm{F}$ & 05 & West & (A) & 07/19/03 & WAP & I & P03149:01 & Tank condition had not changed. \\
\hline
\end{tabular}




\begin{tabular}{|c|c|c|c|c|c|c|c|}
\hline \multirow{2}{*}{$\frac{\text { AREA }}{F}$} & \multirow{2}{*}{$\begin{array}{c}\begin{array}{c}\text { TANK OR } \\
\text { ANCILLARY }\end{array} \\
05\end{array}$} & $\frac{\text { ACCESS OPENING }}{(A \text { OR I) }}$ & \multirow{2}{*}{$\frac{\text { DATE }}{03 / 24 / 03}$} & \multicolumn{3}{|c|}{$\frac{\text { INSPECTION METHOD }}{\text { IDENTIFICATION NUMBER }}$} & \multirow{2}{*}{$\begin{array}{l}\qquad \text { REMARKS } \\
\text { Inspection documented conditions } \\
\text { beneath the riser. No unusual } \\
\text { conditions were observed. }\end{array}$} \\
\hline & & $01 \quad$ (I) & & CCTV & I & 938 & \\
\hline $\mathrm{F}$ & 05 & $03 \quad(\mathrm{I})$ & $03 / 24 / 03$ & CCTV & I & 917 & $\begin{array}{l}\text { Inspection documented conditions } \\
\text { beneath the riser. No unusual } \\
\text { conditions were observed. } \\
\text { Abandoned steel tapes were } \\
\text { observed directly below the riser. }\end{array}$ \\
\hline$F$ & 05 & $03 \quad(\mathrm{I})$ & $12 / 18 / 03$ & CCTV & I & 1046 & $\begin{array}{l}\text { The HLLCP was properly } \\
\text { deployed. }\end{array}$ \\
\hline$F$ & 05 & $04 \quad(\mathrm{I})$ & $03 / 24 / 03$ & CCTV & I & 917 & $\begin{array}{l}\text { Inspection documented conditions } \\
\text { beneath the riser. No unusual } \\
\text { conditions were observed. }\end{array}$ \\
\hline$F$ & 05 & $06 \quad$ (I) & 08/01/03 & CCTV & I & 995 & $\begin{array}{l}\text { Inspection documented conditions } \\
\text { in and beneath riser prior to } \\
\text { transfer jet removal. No unusual } \\
\text { conditions were observed. }\end{array}$ \\
\hline
\end{tabular}

Inspection documented conditions beneath the riser. No unusual conditions were observed.

Inspection documented transfer jet configuration.

CCTV was used to monitor mining activities beneath riser 6 . The mining rig appeared to be contacting something below the liquid surface which was most likely a bottom horizontal cooling coil. No other unusual conditions were observed.

Inspection documented conditions beneath the riser. No unusual conditions were observed.

CCTV was used to deploy a new HLLCP. The HLLCP was properly deployed. No unusual conditions were observed.

Inspection was made to locate cross braces on the transfer jet. One brace was located approximately 3 feet from the bottom of the jet.

Tank condition had not changed. 


\begin{tabular}{|c|c|c|c|c|}
\hline AREA & $\begin{array}{l}\text { TANK OR } \\
\text { ANCILLARY }\end{array}$ & $\frac{\text { ACCESS OPENING }}{(A \text { OR I) }}$ & DATE & $\frac{\text { INSPECTION METHOD }}{\text { IDENTIFICATION NUMBER }}$ \\
\hline $\mathrm{F}$ & 06 & East (A) & 07/19/03 & CCTV \\
\hline
\end{tabular}

\section{REMARKS}

The magnetically mounted thermocouple was deployed per Structural Integrity Data Sheet T-DS-G-00010.

$\begin{array}{ccccc}\text { North (A) } & 07 / 16 / 03 & \text { DP } & \text { / } & \text { P03143:01-23 } \\ \text { North (A) } & 07 / 19 / 03 & \text { CCTV } & \text { / } & 926\end{array}$

South (A) $\quad 07 / 16 / 03$

WAP / P03148:01

CCTV I 926

South (A) $\quad 07 / 19 / 03$

$\mathrm{F}$

06

06

06

$\mathrm{F}$

$\mathrm{F}$

06

02 (I)

07/10/03

CCTV

990

West (A)

07/16/03

WAP / P03148:02

990

CCTV

1

(1)

04 (I)

07/10/03
CCTV I

990
Tank condition had not changed.

The conductivity probe was properly deployed per setpoint document J-JX-G-0001.

Tank condition had not changed.

The conductivity probe was document J-JX-G-0001.

Tank condition had not changed.

Inspection documented the conditions of all visible tank risers and any obstructions beneath the riser openings. Liquid was observed throughout the tank. No unusual conditions were observed.

Inspection documented the conditions of all visible tank risers and any obstructions beneath the riser openings. Liquid was observed throughout the tank. Water was observed dripping around the center riser. No other unusual conditions were observed.

Inspection documented the conditions of all visible tank risers and any obstructions beneath the riser openings. The thermowell was installed in the riser. Liquid was observed throughout the tank. No unusual conditions were observed.

Inspection documented the conditions of all visible tank risers and any obstructions beneath the riser openings. Liquid was observed throughout the tank. The HLLCP was properly positioned. No unusual conditions were observed. properly deployed per setpoint 


\section{TANK OR \\ AREA}

$\mathrm{F}$

06

$\frac{\frac{\text { ACCESS OPENING }}{\text { (A OR I) }}}{05 \quad \text { (I) }} \frac{\text { DATE }}{07 / 15 / 03}$

INSPECTION METHOD IDENTIFICATION NUMBER

CCTV / 992

CCTV I 990

$06 \quad(I)$

$11 / 04 / 03$

(9)

$\mathrm{F}$

06

$07 \quad$ (I)

07/15/03

CCTV I

992

CCTV

I

875

07/15/03

CCTV

992

\section{REMARKS}

Inspection documented the conditions of all visible tank risers and any obstructions beneath the riser openings. The reel tape, HLLCP and dip tubes were installed in the riser. Liquid was observed throughout the tank. No unusual conditions were observed.

Inspection documented the conditions of the riser piping, jet and any obstructions beneath the riser. No obstructions were observed, and the exterior of the jet piping from the liquid surface to the riser was normal.

Inspection documented the conditions of the tank and any obstructions beneath the riser opening. No obstructions or unusual conditions were observed.

Inspection documented the conditions of the tank and any obstructions beneath all visible riser openings. Liquid was observed throughout the tank. No unusual conditions were observed.

CCTV was used to deploy a new HLLCP. The HLLCP was properly deployed.

Inspection documented the conditions of the tank and any obstructions beneath all visible riser openings. Water was observed dripping around the riser. No other unusual conditions were observed.

DP

/ P03123:01-22

CCTV / 926

North (A)

07/07/03
F

F
07

07

07
South (A)

South
06/30/03

07/07/03
WAP / P03125:01

CCTV / 926
Tank condition was normal.

The conductivity probe was properly deployed per setpoint document J-JX-G-0001.

Tank condition was normal.

The conductivity probe was properly deployed per setpoint document J-JX-G-0001. 


\begin{tabular}{|c|c|c|c|c|c|c|c|c|}
\hline \multirow{2}{*}{$\frac{\text { AREA }}{F}$} & \multirow{2}{*}{$\begin{array}{c}\begin{array}{c}\text { TANK OR } \\
\text { ANCILLARY }\end{array} \\
07\end{array}$} & \multicolumn{2}{|c|}{$\frac{\text { ACCESS OPENING }}{(A \text { OR I) }}$} & \multirow{2}{*}{$\frac{\text { DATE }}{06 / 30 / 03}$} & \multicolumn{3}{|c|}{$\begin{array}{l}\text { INSPECTION METHOD } \\
\text { IDENTIFICATION NUMBER }\end{array}$} & \multirow{2}{*}{$\frac{\text { REMARKS }}{\text { Tank condition was normal. }}$} \\
\hline & & West & (A) & & WAP & l & P03125:02 & \\
\hline $\mathrm{F}$ & 07 & West & (A) & 07/07/03 & CCTV & l & 926 & $\begin{array}{l}\text { The magnetically mounted } \\
\text { thermocouple was deployed per } \\
\text { Structural Integrity Data Sheet } \\
\text { T-DS-G-00010. }\end{array}$ \\
\hline $\mathrm{F}$ & 07 & Center & (I) & 02/09/03 & CCTV & l & 917 & $\begin{array}{l}\text { Inspection revealed liquid with } \\
\text { foam floating on the surface. } \\
\text { Sludge was visible around the } \\
\text { transfer pump and near the tank } \\
\text { wall. The reel tape was coated } \\
\text { with foam. }\end{array}$ \\
\hline $\mathrm{F}$ & 07 & Center & (I) & 04/03/03 & CCTV & l & 945 & $\begin{array}{l}\text { Inspection documented tank } \\
\text { conditions after transfer to Tank } \\
51 \text {. Sludge and liquid were } \\
\text { observed throughout the tank. }\end{array}$ \\
\hline $\mathrm{F}$ & 07 & Center & (I) & $05 / 11 / 03$ & CCTV & l & 945 & $\begin{array}{l}\text { Inspection documented tank } \\
\text { conditions after transfer to Tank } \\
51 \text {. Sludge and liquid were } \\
\text { observed throughout the tank. }\end{array}$ \\
\hline $\mathrm{F}$ & 07 & Center & (I) & 06/26/03 & CCTV & l & 962 & $\begin{array}{l}\text { Inspection documented tank } \\
\text { conditions. Sludge was observed } \\
\text { above the liquid, and a mound of } \\
\text { sludge was observed near the } \\
\text { tank wall. No unusual conditions } \\
\text { were observed. }\end{array}$ \\
\hline $\mathrm{F}$ & 08 & East & (A) & 06/28/03 & WAP & l & P03129:01 & Tank condition was normal. \\
\hline $\mathrm{F}$ & 08 & East & (A) & 06/29/03 & CCTV & l & 926 & $\begin{array}{l}\text { The magnetically mounted } \\
\text { thermocouple was deployed per } \\
\text { Structural Integrity Data Sheet } \\
\text { T-DS-G-00010. }\end{array}$ \\
\hline $\mathrm{F}$ & 08 & North & (A) & 06/28/03 & WAP & I & P03129:02 & Tank condition was normal. \\
\hline $\mathrm{F}$ & 08 & North & (A) & 06/29/03 & CCTV & l & 926 & $\begin{array}{l}\text { The conductivity probe was } \\
\text { properly deployed per setpoint } \\
\text { document J-JX-G-0001. }\end{array}$ \\
\hline $\mathrm{F}$ & 08 & South & (A) & $06 / 29 / 03$ & CCTV & l & 926 & $\begin{array}{l}\text { The conductivity probe was } \\
\text { properly deployed per setpoint } \\
\text { document J-JX-G-0001. }\end{array}$ \\
\hline $\mathrm{F}$ & 08 & South & (A) & $07 / 02 / 03$ & DP & l & P03126:01-22 & Tank condition was normal. \\
\hline $\mathrm{F}$ & 08 & West & (A) & 06/28/03 & WAP & l & P03129:03 & Tank condition was normal. \\
\hline $\mathrm{H}$ & 09 & South & (A) & $02 / 04 / 03$ & CCTV & l & 914 & $\begin{array}{l}\text { The conductivity probe was } \\
\text { properly deployed per setpoint } \\
\text { document J-JX-G-0001. }\end{array}$ \\
\hline
\end{tabular}




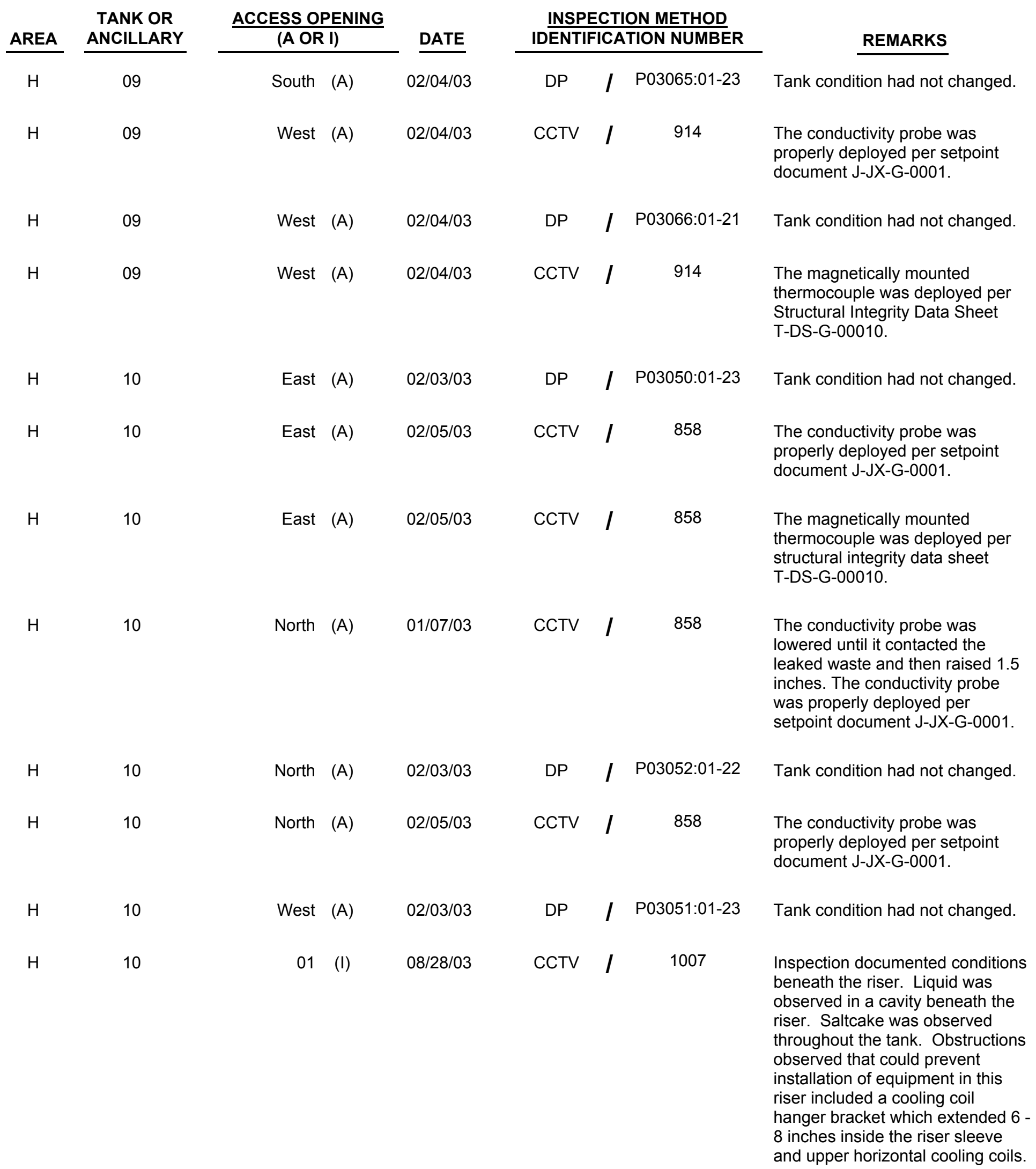




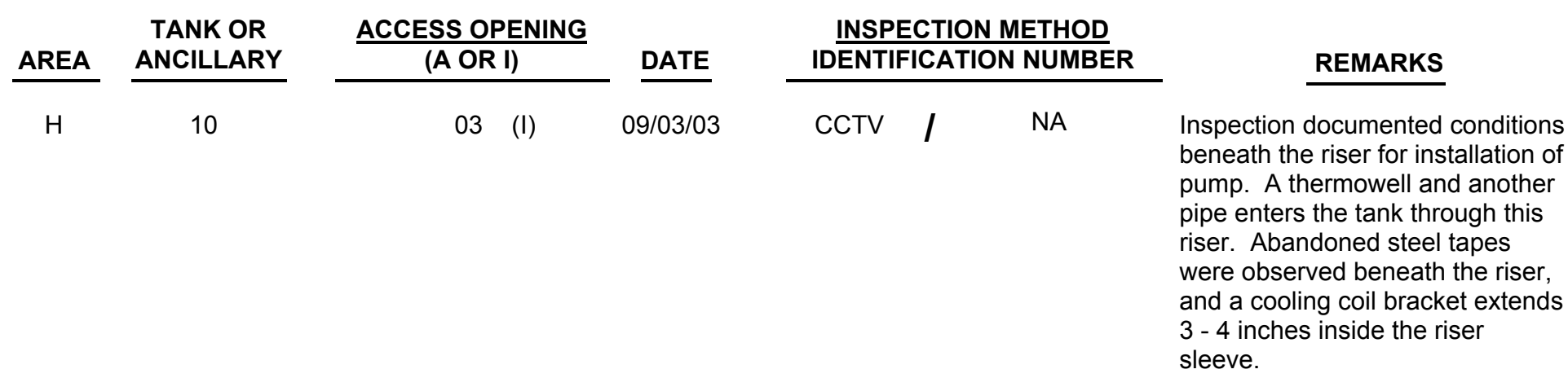

$\mathrm{H}$

$\mathrm{H}$

$\mathrm{H}$

$\mathrm{H}$

$\mathrm{H}$

$\mathrm{H}$

$\mathrm{H}$

$\mathrm{H}$

$\mathrm{H}$

$\mathrm{H}$
10

10

11

11

11

11

11

11

11

11
$04 \quad$ (I)

$08 / 28 / 03$

$08 \quad(I)$

$08 / 28 / 03$

East

(A)

North (A)

02/03/03

North (A)

02/03/03

South (A)

02/03/03

South (A)

02/03/03

West

(A)

02/03/03

West (A)

02/10/03

CCTV

914

03/11/03

$01 \quad$ (I)

(I)

$03 / 11 / 03$

DP

DP

DP

CCTV / $\quad 914$

DP
CCTV / 1007

CCTV / 1007

/ P03049:01-22

CCTV / 914

P03048:01-23

$\mathrm{P} 03036: 01-23$

Tank condition had not changed.

The magnetically mounted thermocouple was deployed per Structural Integrity Data Sheet T-DS-G-00010.

Inspection verified that no obstructions existed beneath the riser which would prevent slurry pump installation. 


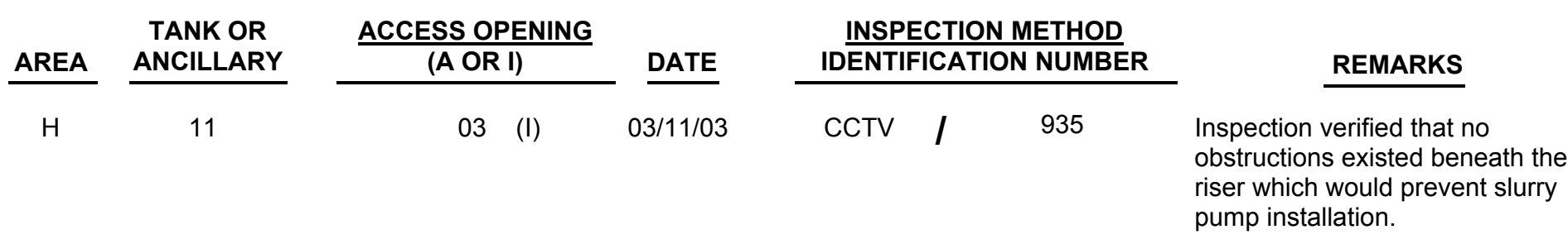

$\mathrm{H}$

11

11

11

$\mathrm{H}$

$\mathrm{H}$

$\mathrm{H}$

$H$

$\mathrm{H}$

$\mathrm{H}$

$\mathrm{H}$

$\mathrm{H}$

$\mathrm{H}$

$\mathrm{H}$

12

11

11

12

12

12

12

12
$05 \quad$ (I)

$03 / 11 / 03$

$05 \quad$ (I)

05/19/03

CCTV

935

$06 \quad$ (I)

03/11/03

CCTV

935

$06 \quad$ (I)

09/08/03

$08 \quad(\mathrm{I})$

03/13/03

East (A)

02/14/03

East (A)

02/14/03

CCTV I

1011

CCTV / $\quad 935$
South

(A)

02/14/03

South (A) 10/26/03

North (A) 02/14/03

North (A) 10/26/03

West

(A)

02/14/03

Inspection verified that no obstructions existed beneath the riser which would prevent slurry pump installation.

Inspection verified that no obstructions existed beneath the riser which would prevent slurry pump installation.

Inspection was made to document any obstructions beneath the riser which would prevent the installation of a slurry pump. A pair of cooling coils were observed beneath the riser opening.

CCTV was used to facilitate remote installation of a "go / no go" device. A cooling coil beneath the riser prevented the device from completely entering the tank and would prevent pump installation. any obstructions beneath the riser which would prevent the installation of a slurry pump. No obstructions were observed.

Tank condition had not changed.

The magnetically mounted thermocouple was deployed per Structural Integrity Data Sheet T-DS-G-00010.

DP / P03082:01-22 Tank condition had not changed.

CCTV / $1008 \quad$ The conductivity probe was properly deployed per setpoint document J-JX-G-0001.

DP / P03083:01-20 Tank condition had not changed.

CCTV / $1008 \quad$ The conductivity probe was properly deployed per setpoint document J-JX-G-0001.

DP / P03081:01-22 Tank condition had not changed. 


\section{TANK OR \\ AREA}

$\mathrm{H}$

$\frac{\text { ACCESS OPENING }}{\text { (A OR I) }}$

$01 \quad(\mathrm{I})$ DATE

$10 / 27 / 03$
INSPECTION METHOD IDENTIFICATION NUMBER
REMARKS

CCTV / 1007

Inspection was performed to document conditions beneath the riser and determine if any obstructions exist that may prevent the installation of a slurry pump. A flat bar, most likely attached to a cooling coil, was observed beneath the riser. The waste was dry salt/sludge. No other unusual conditions were observed.

CCTV / 1007

$10 / 27 / 03$

1007

Inspection was performed to document conditions beneath the riser and determine if any obstructions exist that may prevent the installation of a slurry pump. A flat bar, attached to a cooling coil, was observed beneath the riser. The waste was dry salt/sludge. No other unusual conditions were observed.

CCTV

1007

Inspection was performed to document conditions beneath the riser and determine if any obstructions exist that may prevent the installation of a slurry pump. A flat bar, most likely attached to a cooling coil, was observed beneath the riser. The waste was dry salt/sludge. No other unusual conditions were observed.

Inspection was performed to document conditions beneath the riser and determine if any obstructions exist that may prevent the installation of a slurry pump. No obstructions were observed; however, abandoned

steel tapes were observed beneath the riser and five broken cooling coil hangers were observed. The waste was dry

Inspection was performed to document conditions beneath the riser. Abandoned steel tapes were observed beneath the riser. The waste was dry salt/sludge. No unusual conditions were observed. 


\begin{tabular}{|c|c|c|c|c|c|c|c|}
\hline \multirow{2}{*}{$\frac{\text { AREA }}{H}$} & \multirow{2}{*}{$\begin{array}{c}\text { TANK OR } \\
\text { ANCILLARY } \\
13\end{array}$} & $\frac{\text { ACCESS OPENING }}{(A \text { OR I) }}$ & \multirow{2}{*}{$\frac{\text { DATE }}{02 / 24 / 03}$} & \multicolumn{3}{|c|}{$\begin{array}{l}\text { INSPECTION METHOD } \\
\text { IDENTIFICATION NUMBER }\end{array}$} & \multirow{2}{*}{$\begin{array}{l}\qquad \text { REMARKS } \\
\text { The conductivity probe was } \\
\text { properly deployed per setpoint } \\
\text { document J-JX-G-0001. }\end{array}$} \\
\hline & & LDB-02 & & CCTV & l & 908 & \\
\hline $\mathrm{H}$ & 13 & 010 & 09/09/03 & WAP & I & P03188:06 & Tank condition had not changed. \\
\hline $\mathrm{H}$ & 13 & 032 & 09/09/03 & WAP & l & P03188:05 & Tank condition had not changed. \\
\hline $\mathrm{H}$ & 13 & $055 \quad(A)$ & 09/09/03 & WAP & l & P03188:04 & Tank condition had not changed. \\
\hline $\mathrm{H}$ & 13 & 071 (A) & 09/09/03 & WAP & I & P03188:03 & Tank condition had not changed. \\
\hline $\mathrm{H}$ & 13 & $107 \quad(\mathrm{~A})$ & 09/09/03 & WAP & l & P03188:02 & Tank condition had not changed. \\
\hline $\mathrm{H}$ & 13 & 151 (A) & 09/09/03 & WAP & l & P03188:09 & Tank condition had not changed. \\
\hline $\mathrm{H}$ & 13 & 175 (A) & 09/09/03 & DP & I & P03197:01-22 & Tank condition had not changed. \\
\hline $\mathrm{H}$ & 13 & 207 & 09/09/03 & DP & I & P03196:01-22 & Tank condition had not changed. \\
\hline $\mathrm{H}$ & 13 & 228 & 09/09/03 & WAP & I & P03188:08 & Tank condition had not changed. \\
\hline $\mathrm{H}$ & 13 & East (A) & $09 / 09 / 03$ & DP & I & P03198:01-23 & Tank condition had not changed. \\
\hline
\end{tabular}

CCTV / 1008

The conductivity probe was properly deployed per setpoint document J-JX-G-0001.

The magnetically mounted thermocouple was deployed per Structural Integrity Data Sheet T-DS-G-00010.

Tank condition had not changed.

The conductivity probe was properly deployed per setpoint document J-JX-G-0001.

Tank condition had not changed. An anomaly observed approximately 5 feet above the middle girth weld will be investigated.

CCTV was used to investigate an anomaly observed on a photographic inspection performed on $9 / 9 / 03$. The spot observed was not visible and was most likely a floating dust particle captured in the still photograph. 


\begin{tabular}{|c|c|c|c|c|c|c|c|c|}
\hline \multirow{2}{*}{$\frac{\text { AREA }}{H}$} & \multirow{2}{*}{$\begin{array}{c}\begin{array}{c}\text { TANK OR } \\
\text { ANCILLARY }\end{array} \\
14\end{array}$} & \multicolumn{2}{|c|}{$\frac{\text { ACCESS OPENING }}{(A \text { OR I) }}$} & \multirow{2}{*}{$\frac{\text { DATE }}{09 / 14 / 03}$} & \multicolumn{3}{|c|}{$\begin{array}{l}\text { INSPECTION METHOD } \\
\text { IDENTIFICATION NUMBER }\end{array}$} & \multirow{2}{*}{$\frac{\text { REMARKS }}{\text { Tank condition had not changed. }}$} \\
\hline & & 013 & (A) & & DP & I & P03207:01-22 & \\
\hline $\mathrm{H}$ & 14 & 032 & (A) & 09/14/03 & WAP & l & P03199:01 & Tank condition had not changed. \\
\hline $\mathrm{H}$ & 14 & 065 & (A) & 09/14/03 & WAP & l & P03199:02 & Tank condition had not changed. \\
\hline $\mathrm{H}$ & 14 & 108 & (A) & 09/14/03 & WAP & l & P03199:03 & Tank condition had not changed. \\
\hline $\mathrm{H}$ & 14 & 118 & (A) & 09/14/03 & DP & l & P03208:01-22 & Tank condition had not changed. \\
\hline $\mathrm{H}$ & 14 & 125 & (A) & 09/14/03 & WAP & l & P03199:04 & Tank condition had not changed. \\
\hline $\mathrm{H}$ & 14 & 151 & (A) & 09/14/03 & DP & l & P03210:01-22 & Tank condition had not changed. \\
\hline $\mathrm{H}$ & 14 & 170 & (A) & 09/14/03 & DP & l & P03209:01-22 & Tank condition had not changed. \\
\hline $\mathrm{H}$ & 14 & 207 & (A) & 09/14/03 & DP & l & P03211:01-22 & Tank condition had not changed. \\
\hline $\mathrm{H}$ & 14 & 235 & (A) & 09/14/03 & DP & l & P03212:01-22 & Tank condition had not changed. \\
\hline $\mathrm{H}$ & 14 & 259 & (A) & 09/14/03 & DP & l & P03213:01-22 & Tank condition had not changed. \\
\hline
\end{tabular}

CCTV / 1008

The conductivity probe was properly deployed per setpoint document J-JX-G-0001.

Tank condition had not changed.

The magnetically mounted thermocouple was deployed per Structural Integrity Data Sheet T-DS-G-00010.

The conductivity probe was properly deployed per setpoint document J-JX-G-0001.

Tank condition had not changed.

Tank condition had not changed.

The conductivity probe in the South riser was lifted so that it could be viewed and then lowered back down beneath the ventilation duct on the annulus floor. The conductivity probe was properly deployed per setpoint document J-JX-G-0001. 


\begin{tabular}{|c|c|c|c|c|c|c|c|c|}
\hline \multirow{2}{*}{$\frac{\text { AREA }}{\mathrm{H}}$} & \multirow{2}{*}{$\begin{array}{c}\text { TANK OR } \\
\text { ANCILLARY } \\
15\end{array}$} & \multicolumn{2}{|c|}{$\frac{\text { ACCESS OPENING }}{(A \text { OR I) }}$} & \multirow{2}{*}{$\frac{\text { DATE }}{09 / 12 / 03}$} & \multicolumn{3}{|c|}{ IDSPECTION METHOD } & \multirow{2}{*}{$\frac{\text { REMARKS }}{\text { Tank condition had not changed. }}$} \\
\hline & & 055 & (A) & & WAP & I & P03189:05 & \\
\hline $\mathrm{H}$ & 15 & 071 & (A) & 09/12/03 & WAP & I & P03189:04 & Tank condition had not changed. \\
\hline $\mathrm{H}$ & 15 & 107 & (A) & 09/12/03 & DP & l & P03202:01-22 & Tank condition had not changed. \\
\hline $\mathrm{H}$ & 15 & 117 & (A) & 09/13/03 & CCTV & I & 1008 & $\begin{array}{l}\text { The conductivity probe was } \\
\text { properly deployed per setpoint } \\
\text { document J-JX-G-0001. }\end{array}$ \\
\hline $\mathrm{H}$ & 15 & 137 & (A) & $11 / 02 / 03$ & DP & l & P03203:01-22 & Tank condition had not changed. \\
\hline $\mathrm{H}$ & 15 & 171 & (A) & $09 / 12 / 03$ & DP & l & P03204:01-22 & $\begin{array}{l}\text { Tank condition had not changed. } \\
\text { A small amount of waste had } \\
\text { dislodged from a previously } \\
\text { identified leaksite on the wall, } \\
\text { increasing the amount of waste on } \\
\text { the annulus floor. }\end{array}$ \\
\hline $\mathrm{H}$ & 15 & 182 & (A) & 09/12/03 & DP & l & P03205:01-22 & $\begin{array}{l}\text { Tank condition had not changed. } \\
\text { Leaked waste on the annulus floor } \\
\text { had been reconfigured due to the } \\
\text { inleakage of water. }\end{array}$ \\
\hline $\mathrm{H}$ & 15 & 207 & (A) & $09 / 12 / 03$ & WAP & I & P03189:07 & Tank condition had not changed. \\
\hline $\mathrm{H}$ & 15 & 223 & (A) & $09 / 12 / 03$ & WAP & l & P03189:06 & Tank condition had not changed. \\
\hline $\mathrm{H}$ & 15 & 242 & (A) & 09/13/03 & CCTV & l & 1008 & $\begin{array}{l}\text { The magnetically mounted } \\
\text { thermocouple was deployed per } \\
\text { Structural Integrity Data Sheet } \\
\text { T-DS-G-00010. }\end{array}$ \\
\hline $\mathrm{H}$ & 15 & East & (A) & $09 / 12 / 03$ & WAP & l & P03189:08 & Tank condition had not changed. \\
\hline $\mathrm{H}$ & 15 & North & (A) & 09/12/03 & WAP & l & P03189:03 & Tank condition had not changed. \\
\hline $\mathrm{H}$ & 15 & South & (A) & $09 / 12 / 03$ & DP & l & P03200:01-22 & Tank condition had not changed. \\
\hline $\mathrm{H}$ & 15 & West & (A) & 09/12/03 & WAP & I & P03189:01 & Tank condition had not changed. \\
\hline $\mathrm{H}$ & $15 / 16$ & VB & & $03 / 21 / 03$ & CCTV & l & 908 & $\begin{array}{l}\text { Inspection verified that the } \\
\text { conductivity probe was properly } \\
\text { deployed. }\end{array}$ \\
\hline $\mathrm{H}$ & 16 & 035 & (A) & 09/13/03 & DP & I & P03191:01-22 & $\begin{array}{l}\text { Tank condition had not changed. } \\
\text { Leaked waste on the annulus floor } \\
\text { had been reconfigured due to the } \\
\text { inleakage of water. }\end{array}$ \\
\hline $\mathrm{H}$ & 16 & 118 & (A) & 09/13/03 & DP & I & P03192:01-22 & Tank condition had not changed. \\
\hline
\end{tabular}




\begin{tabular}{|c|c|c|c|c|c|c|c|}
\hline \multirow{2}{*}{$\frac{\text { AREA }}{H}$} & \multirow{2}{*}{$\begin{array}{c}\begin{array}{c}\text { TANK OR } \\
\text { ANCILLARY }\end{array} \\
16\end{array}$} & $\frac{\text { ACCESS OPENING }}{(A \text { OR I) }}$ & \multirow{2}{*}{$\frac{\text { DATE }}{09 / 13 / 03}$} & \multicolumn{3}{|c|}{$\frac{\text { INSPECTION METHOD }}{\text { IDENTIFICATION NUMBER }}$} & \multirow{2}{*}{$\begin{array}{l}\qquad \text { REMARKS } \\
\text { Tank condition had not changed. } \\
\text { Leaked waste on the annulus flo } \\
\text { had been reconfigured due to } \\
\text { water inleakage. }\end{array}$} \\
\hline & & 207 (A) & & DP & I & P03193:01-21 & \\
\hline $\mathrm{H}$ & 16 & $262(\mathrm{~A})$ & $09 / 13 / 03$ & DP & l & P03194:01-22 & Tank condition had not changed. \\
\hline $\mathrm{H}$ & 16 & East $(\mathrm{A})$ & $09 / 13 / 03$ & WAP & l & P03190:02 & Tank condition had not changed. \\
\hline $\mathrm{H}$ & 16 & West $(A)$ & $09 / 13 / 03$ & WAP & l & P03190:01 & Tank condition had not changed. \\
\hline $\mathrm{F}$ & 18 & Center (I) & $02 / 19 / 03$ & CCTV & l & 921 & $\begin{array}{l}\text { Inspection revealed steel tapes } \\
\text { and hoses beneath the ADMP. } \\
\text { Exposed sludge was observed. }\end{array}$ \\
\hline
\end{tabular}

Inspection documented the conditions beneath the ADMP. Several abandoned steel tapes were observed below the liquid level and directly beneath the ADMP.

Inspection was made to document conditions beneath the ADMP after steel tapes were removed from beneath the pump.

Inspection was made to document conditions of the ADMP screens and any obstructions located beneath the pump. The ADMP sidewall screens appear normal.

CCTV was used to facilitate the replacement of the HLLCP. The HLLCP was properly deployed.

Inspection was made to document the waste level and sludge in the tank. The waste surface was mostly liquid with foam on the surface. A mound of exposed sludge was observed near the tank wall.

Inspection below the ADMP and slurry pump revealed no unusual conditions. No exposed sludge was observed.

Inspection documented the waste surface and HLLCP. No unusual conditions were observed. 


\section{TANK OR \\ AREA ANCILLARY}

$\mathrm{F}$

18 (A OR I)

DATE

Center (I)

06/19/03

18

F

F

18

Center (I)

$07 / 07 / 03$

$\mathrm{F}$
$10 / 03 / 03$

Center (I)

18
$02 / 12 / 03$

NE (I)

CCTV

895
18
NE (I)

02/19/03
CCTV

986

INSPECTION METHOD

IDENTIFICATION NUMBER

986

970

CCTV / 1018

\section{REMARKS}

Inspection revealed abandoned steel tapes and tubing had become entwined in the screen at the bottom of the ADMP.

Inspection documented tank conditions and any observable solids.

Inspection documented conditions of the ADMP screen. The screen had abandoned steel tapes and rubber tubing entwined in the screen. Steel tapes and tubing was also observed beneath the pump below the liquid surface.

Tank steel wall and concrete dome were normal. A few surface voids and stains were observed in the concrete dome. Exposed mounds were observed near the

South and North walls with a ridge exposed in the SSE portion of the tank. A small mound was visible beneath the center riser. The waste was wet, and the exposed solids were wet or damp.

Abandoned steel tapes, hoses

and other extraneous items were observed on the bottom of the tank. A portion of the steel floor is visible evidenced by a $3 / 8$ inch lifting plate attached to the tank bottom. Evaluation is documented

Inspection documented the conditions in the tank. Abandoned steel tapes were observed beneath the liquid surface. Hoses and tubing were observed on the surface. Sludge was observed above the liquid surface.

Inspection documented the conditions beneath the ADMP. The waste was liquid with exposed sludge in the northeast portion of the tank. 


$\begin{array}{cccc}\text { TANK OR } & \begin{array}{c}\text { ACCESS OPENING } \\ \text { AREA }\end{array} & \text { INCILLARY } & \text { INSPECTION METHOD } \\ \text { AN ORI) } & \text { DATE } & \text { IDENTIFICATION NUMBER }\end{array}$

$\mathrm{F}$
Center (I) 10/04/03
877

\section{REMARKS}

Tank concrete dome condition was normal, and the carbon steel liner condition had not changed. Solids were observed on the 5 inch stiffener bands and adhering to the tank walls. Exposed solids were observed, and liquid was covering approximately $50 \%$ of the tank bottom. Abandoned steel tapes and other extraneous items were observed on the bottom of the tank. Evaluation is documented in document CBU-LTS-2003-00171.
The conductivity probe was lowered until it contacted the bottom of the valve box, raised 1/4 inch and then secured at that elevation. The conductivity probe was properly deployed per setpoint document J-JX-G-0001.

Tank steel wall and concrete dome conditions were normal. Water intrusion and calciferous deposits at the interface of the tank wall and concrete dome had increased slightly. Some small surface voids were observed on the concrete dome. The surface of the waste was covered with a film. The HLLCP was properly deployed.

The conductivity probe was properly deployed per setpoint document J-JX-G-0001.

The conductivity probe was lowered until it contacted the bottom of the valve box, raised $1 / 4$ inch and then secured at that elevation. The conductivity probe was properly deployed per setpoint document J-JX-G-0001.

Inspection verified that the conductivity probe was properly deployed.

Inspection of the reel tape revealed no unusual conditions. The waste surface was covered with a film and rust colored "splotches" throughout the tank. 


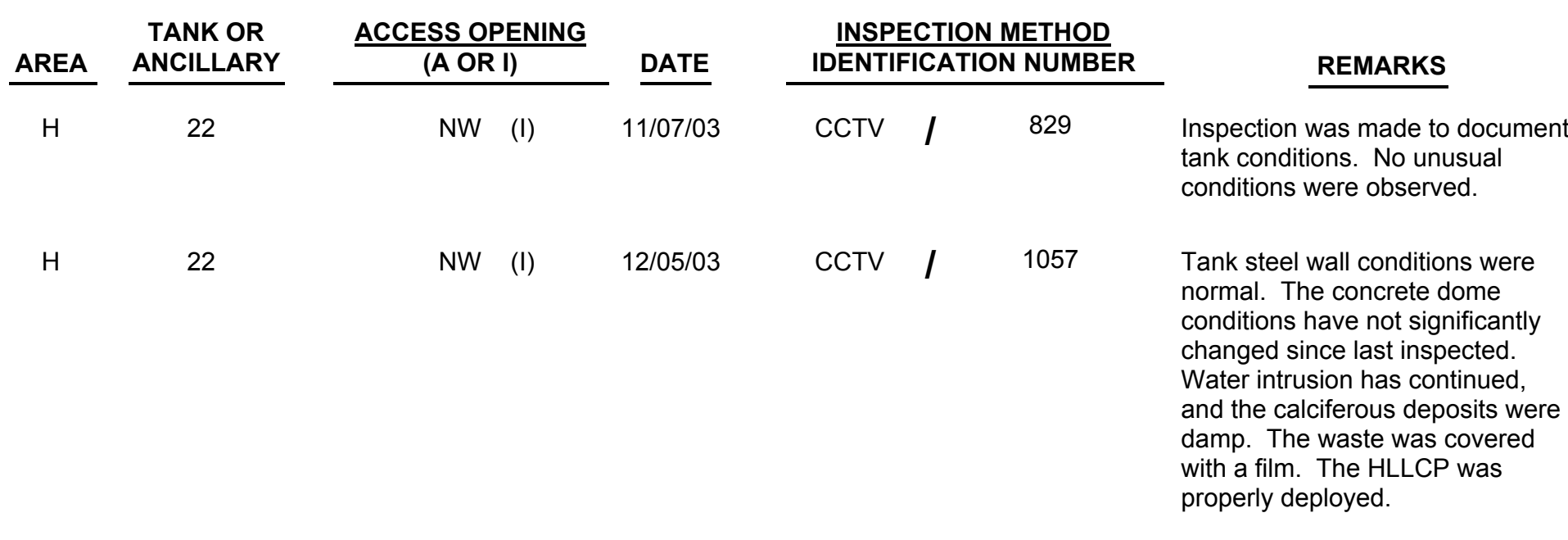

$\mathrm{H}$

$\mathrm{H}$
23

24

25

25

25

25

25

25

25

25
SW (I)

$11 / 09 / 03$

CCTV I

834

SW (I)

$11 / 09 / 03$

CCTV I

815

LDB-03

09/10/03

CCTV

964

09/30/03

CCTV

964

LDB-03

A-02 (A)

A-02 (A)

A-03 (A)

A-03 (A) 02/25/03

A-04 (A)

02/10/03
02/10/03

02/25/03

02/10/03

CCTV

914

02/10/03

WAP

CCTV

914

WAP

P03099:01

WAP

I

P03099:02

$\begin{array}{lll}\text { CCTV I } & 914\end{array}$
Tank steel wall and concrete dome conditions were normal. The HLLCP is properly deployed.

Tank steel wall conditions were normal. Inspection of the concrete dome revealed a few surface voids. Stains and marks on the dome were caused by water inleakage. The waste surface was covered with a film and floating solids throughout the tank. The HLLCP was properly deployed.

The conductivity probe was properly deployed per setpoint document J-JX-G-0001.

The conductivity probe was properly deployed per setpoint document J-JX-G-0001. Liquid was observed in the bottom of the LDB causing the probe to be in the alarm mode.

Tank condition was normal.

The conductivity probe was properly deployed per setpoint document J-JX-G-0001.

Tank condition was normal.

The conductivity probe was properly deployed per setpoint document J-JX-G-0001.

Tank condition was normal.

The conductivity probe was properly deployed per setpoint document J-JX-G-0001. 


\begin{tabular}{|c|c|c|c|c|c|c|c|c|}
\hline \multirow{2}{*}{$\frac{\text { AREA }}{F}$} & \multirow{2}{*}{$\begin{array}{c}\begin{array}{c}\text { TANK OR } \\
\text { ANCILLARY }\end{array} \\
25\end{array}$} & \multicolumn{2}{|c|}{$\frac{\text { ACCESS OPENING }}{(A \text { OR I) }}$} & \multirow{2}{*}{$\frac{\text { DATE }}{02 / 10 / 03}$} & \multicolumn{3}{|c|}{$\begin{array}{l}\text { INSPECTION METHOD } \\
\text { IDENTIFICATION NUMBER }\end{array}$} & \multirow{2}{*}{$\frac{\text { REMARKS }}{\text { Tank condition was normal. }}$} \\
\hline & & $\mathrm{A}-04$ & (A) & & WAP & I & P03057:08 & \\
\hline $\mathrm{F}$ & 25 & P-01 & (A) & $02 / 10 / 03$ & WAP & I & P03057:01 & Tank condition was normal. \\
\hline $\mathrm{F}$ & 25 & $\mathrm{P}-02$ & (A) & $02 / 10 / 03$ & WAP & I & P03057:03 & Tank condition was normal. \\
\hline $\mathrm{F}$ & 25 & P-03 & (A) & $02 / 10 / 03$ & DP & I & P03076:01-27 & Tank condition was normal. \\
\hline $\mathrm{F}$ & 25 & P-04 & (A) & 02/10/03 & DP & I & P03075:01-28 & Tank condition was normal. \\
\hline $\mathrm{F}$ & 25 & P-05 & (A) & $02 / 10 / 03$ & WAP & I & P03057:04 & Tank condition was normal. \\
\hline $\mathrm{F}$ & 25 & P-06 & (A) & $02 / 10 / 03$ & WAP & I & P03057:05 & Tank condition was normal. \\
\hline $\mathrm{F}$ & 25 & P-07 & (A) & $02 / 25 / 03$ & WAP & I & P03099:03 & Tank condition was normal. \\
\hline $\mathrm{F}$ & 25 & P-08 & (A) & $02 / 25 / 03$ & WAP & I & P03099:04 & Tank condition was normal. \\
\hline $\mathrm{F}$ & 25 & P-09 & (A) & $02 / 25 / 03$ & WAP & I & P03099:05 & Tank condition was normal. \\
\hline $\mathrm{F}$ & 25 & $P-10$ & (A) & 02/10/03 & WAP & I & P03057:06 & Tank condition was normal. \\
\hline $\mathrm{F}$ & 25 & $P-11$ & (A) & 02/10/03 & DP & I & P03077:01-27 & Tank condition was normal. \\
\hline $\mathrm{F}$ & 25 & $P-12$ & (A) & 02/10/03 & DP & I & P03074:01-27 & Tank condition was normal. \\
\hline $\mathrm{F}$ & 25 & $P-13$ & (A) & 02/10/03 & WAP & I & P03057:09 & Tank condition was normal. \\
\hline $\mathrm{F}$ & 25 & $\mathrm{P}-14$ & (A) & 02/10/03 & WAP & I & P03057:07 & Tank condition was normal. \\
\hline $\mathrm{F}$ & 25 & B-02 & (I) & $10 / 08 / 03$ & CCTV & I & 825 & $\begin{array}{l}\text { Inspection verified operation of } \\
\text { reel tape. Reel tape was } \\
\text { operating properly, and no } \\
\text { obstructions were observed. }\end{array}$ \\
\hline $\mathrm{F}$ & 25 & C-01 & (I) & $09 / 22 / 03$ & CCTV & l & 825 & $\begin{array}{l}\text { Inspection was made to verify that } \\
\text { a siphon break existed in TTJ } \\
\text { connector head. A stem } \\
\text { extending below the connector } \\
\text { head, most likely the siphon } \\
\text { break, was observed. The end of } \\
\text { the stem was not visible; it was } \\
\text { hidden by the jet leg. }\end{array}$ \\
\hline $\mathrm{F}$ & 26 & LDB-01 & & 09/08/03 & CCTV & I & 964 & $\begin{array}{l}\text { The conductivity probe was } \\
\text { properly deployed per setpoint } \\
\text { document J-JX-G-0001. }\end{array}$ \\
\hline $\mathrm{F}$ & 26 & LDB-02 & & $01 / 28 / 03$ & CCTV & I & 908 & $\begin{array}{l}\text { The conductivity probe was } \\
\text { properly deployed per setpoint } \\
\text { document J-JX-G-0001. }\end{array}$ \\
\hline
\end{tabular}




\begin{tabular}{|c|c|c|c|c|c|c|c|}
\hline \multirow{2}{*}{$\frac{\text { AREA }}{F}$} & \multirow{2}{*}{$\begin{array}{c}\text { TANK OR } \\
\text { ANCILLARY } \\
26\end{array}$} & $\frac{\text { ACCESS OPENING }}{\text { (A OR I) }}$ & \multirow{2}{*}{$\frac{\text { DATE }}{01 / 23 / 03}$} & \multicolumn{3}{|c|}{ INSPECTION METHOD } & \multirow{2}{*}{$\begin{array}{l}\qquad \text { REMARKS } \\
\qquad \begin{array}{l}\text { The conductivity probe was } \\
\text { properly deployed per setpoint } \\
\text { document J-JX-G-0001. }\end{array}\end{array}$} \\
\hline & & LDB-04 & & CCTV & l & 908 & \\
\hline $\mathrm{F}$ & 26 & LDB-05 & $01 / 22 / 03$ & CCTV & l & 908 & $\begin{array}{l}\text { The conductivity probe was } \\
\text { properly deployed per setpoint } \\
\text { document J-JX-G-0001. }\end{array}$ \\
\hline $\mathrm{F}$ & 26 & LDB-08 & $01 / 23 / 03$ & CCTV & l & 908 & $\begin{array}{l}\text { The conductivity probe was } \\
\text { properly deployed per setpoint } \\
\text { document J-JX-G-0001. }\end{array}$ \\
\hline $\mathrm{F}$ & 26 & $\mathrm{~A}-01 \quad(\mathrm{~A})$ & $02 / 18 / 03$ & DP & l & P03059:01-27 & Tank condition was normal. \\
\hline $\mathrm{F}$ & 26 & $\mathrm{~A}-02 \quad(\mathrm{~A})$ & $02 / 18 / 03$ & CCTV & l & 914 & $\begin{array}{l}\text { The conductivity probe was } \\
\text { properly deployed per setpoint } \\
\text { document J-JX-G-0001. }\end{array}$ \\
\hline $\mathrm{F}$ & 26 & $\mathrm{~A}-02 \quad(\mathrm{~A})$ & $02 / 18 / 03$ & DP & l & P03058:01-27 & Tank condition was normal. \\
\hline $\mathrm{F}$ & 26 & A-03 (A) & $02 / 18 / 03$ & CCTV & l & 914 & $\begin{array}{l}\text { The conductivity probe was } \\
\text { properly deployed per setpoint } \\
\text { document J-JX-G-0001. }\end{array}$ \\
\hline $\mathrm{F}$ & 26 & $\mathrm{~A}-03 \quad(\mathrm{~A})$ & 02/18/03 & DP & l & P03060:01-28 & Tank condition was normal. \\
\hline $\mathrm{F}$ & 26 & $\mathrm{~A}-04 \quad(\mathrm{~A})$ & $02 / 18 / 03$ & CCTV & l & 914 & $\begin{array}{l}\text { The conductivity probe was } \\
\text { properly deployed per setpoint } \\
\text { document J-JX-G-0001. }\end{array}$ \\
\hline $\mathrm{F}$ & 26 & $\mathrm{~A}-04 \quad(\mathrm{~A})$ & 02/18/03 & DP & l & P03061:01-27 & Tank condition was normal. \\
\hline $\mathrm{F}$ & 26 & $\mathrm{P}-01 \quad(\mathrm{~A})$ & $02 / 18 / 03$ & WAP & l & P03062:01 & Tank condition was normal. \\
\hline $\mathrm{F}$ & 26 & P-02 (A) & $02 / 18 / 03$ & WAP & l & P03062:02 & Tank condition was normal. \\
\hline $\mathrm{F}$ & 26 & P-03 (A) & $02 / 18 / 03$ & WAP & l & P03062:03 & Tank condition was normal. \\
\hline $\mathrm{F}$ & 26 & P-03 (A) & $11 / 05 / 03$ & UT & l & UT-03-012 & $\begin{array}{l}\text { UT scanning was performed on a } \\
\text { vertical strip } 8.5 \text { inches wide for } \\
\text { the entire accessible height of the } \\
\text { primary vessel wall. Analysis of } \\
\text { the data revealed no reportable } \\
\text { thinning, pitting or stress corrosion } \\
\text { cracking. This information is } \\
\text { included in document } \\
\text { WSRC-TR-2004-00166. }\end{array}$ \\
\hline $\mathrm{F}$ & 26 & $\mathrm{P}-04 \quad(\mathrm{~A})$ & $02 / 18 / 03$ & WAP & l & P03062:04 & Tank condition was normal. \\
\hline
\end{tabular}




\begin{tabular}{|c|c|c|c|c|}
\hline & $\begin{array}{l}\text { TANK OR } \\
\text { ANCILLARY }\end{array}$ & $\frac{\text { ACCESS OPENING }}{(A \text { OR I) }}$ & DATE & $\frac{\text { INSPECTION METHOD }}{\text { IDENTIFICATION NUMBER }}$ \\
\hline
\end{tabular}

$\mathrm{F}$

26

P-04 (A)

$11 / 11 / 03$

\section{REMARKS}

UT / UT-03-013
UT scanning was performed on a vertical strip 8.5 inches wide on the bottom 61 inches of the lower plate and the bottom knuckle to make up for area obstructed under P-3. Analysis of the data revealed no reportable thinning, pitting or stress corrosion cracking. This information is included in document WSRC-TR-2004-00166. $\begin{array}{lll}\text { P-05 } & \text { (A) } & 02 / 18 / 03 \\ \text { P-05 } & \text { (A) } & 11 / 20 / 03\end{array}$
26

26
F

$\mathrm{F}$
26

26

\begin{abstract}
P-06
\end{abstract}
(A)

P-06

(A)
02/18/03

$11 / 11 / 03$
WAP / P03062:06

UT / UT-03-014 $\begin{array}{ccc}\text { WAP } & \text { / } & \text { P03062:05 } \\ \text { UT } & \text { / } & \text { UT-03-015 }\end{array}$

Tank condition was normal.

UT scanning was performed on a vertical strip 8.5 inches wide for the entire accessible height of the primary vessel wall. A vertical weld in the top plate,

approximately 13 feet of the top weld of the bottom knuckle plate and the weld between the middle and lower plates was examined for cracking parallel and perpendicular to the weld. Secondary wall plate 4 was scanned for thinning and pitting. A small area of reportable thinning (0.334 inches) was detected on this plate. Analysis of the data revealed no other reportable thinning, pitting or stress corrosion cracking. This information is included in document WSRC-TR-2004-00166.

Tank condition was normal.

UT scanning was performed on a vertical strip 8.5 inches wide on the lower plate and bottom knuckle to make up for area obstructed under P-10. Analysis of the data revealed no reportable thinning, pitting or stress corrosion cracking. This information is included in document WSRC-TR-2004-00166.

Tank condition was normal.

$\begin{array}{lllllll}\text { P-07 } & \text { (A) } & \text { 02/18/03 } & \text { WAP } & \text { I } & \text { P03062:07 } & \text { Tank condition was normal. } \\ \text { P-08 } & \text { (A) } & 02 / 18 / 03 & \text { WAP } & \text { / } & \text { P03062:08 } & \text { Tank condition was normal. } \\ \text { P-09 } & \text { (A) } & 02 / 18 / 03 & \text { WAP } & / & \text { P03062:09 } & \text { Tank condition was normal. } \\ \text { P-10 (A) } & 02 / 18 / 03 & \text { WAP } & / & \text { P03062:10 } & \text { Tank condition was normal. }\end{array}$




\begin{tabular}{|c|c|c|c|c|}
\hline & $\begin{array}{l}\text { TANK OR } \\
\text { ANCILLARY }\end{array}$ & $\frac{\text { ACCESS OPENING }}{(A \text { OR I) }}$ & DATE & $\frac{\text { INSPECTION METHOD }}{\text { IDENTIFICATION NUMBER }}$ \\
\hline
\end{tabular}

$\mathrm{F}$

26
$10 / 23 / 03$
IDENTIFICATION NUMBER

UT I UT-03-010

\section{REMARKS}

UT scanning was performed on a vertical strip 8.5 inches wide for the entire accessible height of the primary vessel wall. Analysis of the data revealed no reportable thinning, pitting or stress corrosion cracking. Secondary wall plates 1,2 , and 3 were scanned for thinning and pitting. This information is included in document WSRC-TR-2004-00166.

$\begin{array}{llll}\text { WAP } & \text { I } & \text { P03062:11 } & \text { Tank condition was normal. } \\ \text { WAP } & \text { I P03062:12 } & \text { Tank condition was normal. }\end{array}$

UT / UT-03-011

UT scanning was performed on a vertical strip 8.5 inches wide for the entire accessible height of the primary vessel wall. Analysis of the data revealed no reportable thinning, pitting or stress corrosion cracking. This information is included in document WSRC-TR-2004-00166.

Tank condition was normal.

WAP / P03062:14 Tank condition was normal.

CCTV / $908 \quad$ The conductivity probe was properly deployed per setpoint document J-JX-G-0001.

LDB-05
04/01/03 CCTV / 908

04/02/03 CCTV / 908

A-01

(A)

02/10/03

$\mathrm{A}-02 \quad(\mathrm{~A})$

02/10/03

WAP

CCTV / 914

02/10/03

A-02 (A)

WAP

WAP / P03071:10

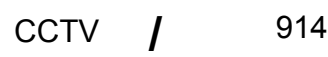

The conductivity probe was properly deployed per setpoint document J-JX-G-0001.

The conductivity probe was properly deployed per setpoint document J-JX-G-0001.

Tank condition was normal.

The conductivity probe was properly deployed per setpoint document J-JX-G-0001.

Tank condition was normal.

A-03 (A) 02/10/03 NTV / 914

The conductivity probe was properly deployed per setpoint document J-JX-G-0001. 


\begin{tabular}{|c|c|c|c|c|c|c|c|c|}
\hline \multirow{2}{*}{$\frac{\text { AREA }}{F}$} & \multirow{2}{*}{$\begin{array}{c}\begin{array}{c}\text { TANK OR } \\
\text { ANCILLARY }\end{array} \\
27\end{array}$} & \multicolumn{2}{|c|}{$\frac{\text { ACCESS OPENING }}{(A \text { OR I) }}$} & \multirow{2}{*}{$\frac{\text { DATE }}{02 / 10 / 03}$} & \multicolumn{3}{|c|}{$\frac{\text { INSPECTION METHOD }}{\text { IDENTIFICATION NUMBER }}$} & \multirow{2}{*}{$\frac{\text { REMARKS }}{\text { Tank condition was normal. }}$} \\
\hline & & A-03 & (A) & & WAP & l & P03071:04 & \\
\hline $\mathrm{F}$ & 27 & A-04 & (A) & $02 / 10 / 03$ & CCTV & I & 914 & $\begin{array}{l}\text { The conductivity probe was } \\
\text { properly deployed per setpoint } \\
\text { document J-JX-G-0001. }\end{array}$ \\
\hline $\mathrm{F}$ & 27 & A-04 & (A) & $02 / 10 / 03$ & WAP & l & P03071:05 & Tank condition was normal. \\
\hline $\mathrm{F}$ & 27 & $\mathrm{P}-01$ & (A) & $02 / 10 / 03$ & WAP & I & P03071:06 & Tank condition was normal. \\
\hline $\mathrm{F}$ & 27 & P-02 & (A) & 02/10/03 & WAP & I & P03071:07 & Tank condition was normal. \\
\hline $\mathrm{F}$ & 27 & P-03 & (A) & $02 / 10 / 03$ & DP & I & P03067:01-27 & Tank condition was normal. \\
\hline $\mathrm{F}$ & 27 & P-04 & (A) & $02 / 10 / 03$ & DP & I & P03068:01-27 & Tank condition was normal. \\
\hline $\mathrm{F}$ & 27 & P-05 & (A) & $02 / 10 / 03$ & WAP & I & P03071:08 & Tank condition was normal. \\
\hline $\mathrm{F}$ & 27 & P-06 & (A) & $02 / 10 / 03$ & WAP & I & P03071:09 & Tank condition was normal. \\
\hline $\mathrm{F}$ & 27 & P-07 & (A) & 02/10/03 & WAP & I & P03071:01 & Tank condition was normal. \\
\hline $\mathrm{F}$ & 27 & P-08 & (A) & $02 / 10 / 03$ & WAP & l & P03071:02 & Tank condition was normal. \\
\hline $\mathrm{F}$ & 27 & P-09 & (A) & 02/10/03 & WAP & l & P03071:03 & Tank condition was normal. \\
\hline $\mathrm{F}$ & 27 & $P-10$ & (A) & $02 / 10 / 03$ & DP & l & P03069:01-27 & Tank condition was normal. \\
\hline $\mathrm{F}$ & 27 & $\mathrm{P}-11$ & (A) & $02 / 10 / 03$ & DP & l & P03070:01-27 & Tank condition was normal. \\
\hline $\mathrm{F}$ & 27 & $\mathrm{P}-12$ & (A) & 02/10/03 & WAP & I & P03071:11 & Tank condition was normal. \\
\hline $\mathrm{F}$ & 27 & $P-13$ & (A) & $02 / 10 / 03$ & WAP & I & P03071:12 & Tank condition was normal. \\
\hline $\mathrm{F}$ & 27 & $P-14$ & (A) & $02 / 10 / 03$ & WAP & l & P03071:13 & Tank condition was normal. \\
\hline $\mathrm{F}$ & 27 & C-01 & (I) & 05/08/03 & CCTV & I & 872 & $\begin{array}{l}\text { Inspection verified that a short } \\
\text { downcomer was installed in the } \\
\text { riser. }\end{array}$ \\
\hline $\mathrm{F}$ & 27 & C-01 & (I) & $12 / 18 / 03$ & CCTV & I & 1055 & $\begin{array}{l}\text { CCTV was used to monitor TTJ } \\
\text { for leaks during transfer. A steam } \\
\text { leak was observed at the stuffing } \\
\text { box. }\end{array}$ \\
\hline $\mathrm{F}$ & 27 & C-03 & (I) & $09 / 17 / 03$ & CCTV & I & 872 & $\begin{array}{l}\text { CCTV documented riser } \\
\text { configuration and verified BFV } \\
\text { was installed. }\end{array}$ \\
\hline
\end{tabular}




\begin{tabular}{|c|c|c|c|c|}
\hline AREA & $\begin{array}{l}\text { TANK OR } \\
\text { ANCILLARY }\end{array}$ & $\frac{\text { ACCESS OPENING }}{(A \text { OR I) }}$ & DATE & $\frac{\text { INSPECTION METHOD }}{\text { IDENTIFICATION NUMBER }}$ \\
\hline $\mathrm{F}$ & 27 & (I) & $12 / 13 / 03$ & CCTV \\
\hline
\end{tabular}

\section{REMARKS}

CCTV was used to monitor the C-01 TTJ for leaks during transfer to Tank 33. Leakage was observed at the steam side stuffing box.

LDB-04

LDB-04

$12 / 30 / 03$

CCTV

1055

09/08/03

CCTV

964

09/10/03

CCTV

964
$\mathrm{A}-01 \quad(\mathrm{~A})$
02/18/03
A-02 (A)
02/18/03

A-02

$\mathrm{A}-02 \quad(A)$

A-03 (A)
$02 / 18 / 03$

02/18/03
DP

CCTV

/ P03085:01-28

914

DP I P03086:01-27

CCTV / 914
CCTV was used to monitor the C-01 TTJ for leaks during transfer to Tank 33. Leakage was observed at the steam side stuffing box.

Inspection documented the conditions of the tank after transfer to Tank 33. The waste was irregular saltcake with pools of liquid observed. The salt accumulation on the cooling coils was minimal. No unusual conditions were observed.

Inspection was made to document conditions in the tank. The waste was irregular saltcake with salt formations on the cooling coils near the waste level. Some liquid was observed, and the C-01 TTJ was in a pool of liquid or just above the liquid.

CCTV documented conditions around bottom of C-01 TTJ. A pool of liquid was observed around the base of the TTJ. A large mound of salt was observed next to the TTJ.

The conductivity probe was properly deployed per setpoint document J-JX-G-0001.

The conductivity probe was properly deployed per setpoint document J-JX-G-0001.

Tank condition was normal.

The conductivity probe was properly deployed per setpoint document J-JX-G-0001.

Tank condition was normal.

The conductivity probe was properly deployed per setpoint document J-JX-G-0001. 


\begin{tabular}{|c|c|c|c|c|c|c|c|c|}
\hline \multirow{2}{*}{$\frac{\text { AREA }}{F}$} & \multirow{2}{*}{$\begin{array}{c}\begin{array}{c}\text { TANK OR } \\
\text { ANCILLARY }\end{array} \\
28\end{array}$} & \multicolumn{2}{|c|}{$\frac{\text { ACCESS OPENING }}{(A \text { OR I) }}$} & \multirow{2}{*}{$\frac{\text { DATE }}{02 / 18 / 03}$} & \multicolumn{3}{|c|}{$\frac{\text { INSPECTION METHOD }}{\text { IDENTIFICATION NUMBER }}$} & \multirow{2}{*}{$\frac{\text { REMARKS }}{\text { Tank condition was normal. }}$} \\
\hline & & $\mathrm{A}-03$ & (A) & & DP & I & P03087:01-27 & \\
\hline $\mathrm{F}$ & 28 & $\mathrm{~A}-04$ & (A) & $02 / 18 / 03$ & CCTV & I & 914 & $\begin{array}{l}\text { The conductivity probe was } \\
\text { properly deployed per setpoint } \\
\text { document J-JX-G-0001. }\end{array}$ \\
\hline $\mathrm{F}$ & 28 & A- 04 & (A) & 02/18/03 & DP & I & P03088:01-27 & Tank condition was normal. \\
\hline $\mathrm{F}$ & 28 & $\mathrm{P}-01$ & (A) & $02 / 18 / 03$ & WAP & I & P03089:01 & Tank condition was normal. \\
\hline $\mathrm{F}$ & 28 & $\mathrm{P}-02$ & (A) & $02 / 18 / 03$ & WAP & I & P03089:02 & Tank condition was normal. \\
\hline $\mathrm{F}$ & 28 & $\mathrm{P}-03$ & (A) & $02 / 18 / 03$ & WAP & I & P03089:03 & Tank condition was normal. \\
\hline $\mathrm{F}$ & 28 & $\mathrm{P}-04$ & (A) & $02 / 18 / 03$ & WAP & I & P03089:04 & Tank condition was normal. \\
\hline $\mathrm{F}$ & 28 & P-05 & (A) & 02/18/03 & WAP & I & P03089:05 & Tank condition was normal. \\
\hline $\mathrm{F}$ & 28 & P-06 & (A) & 02/18/03 & WAP & I & P03089:06 & Tank condition was normal. \\
\hline $\mathrm{F}$ & 28 & P-07 & (A) & $02 / 18 / 03$ & WAP & I & P03089:07 & Tank condition was normal. \\
\hline $\mathrm{F}$ & 28 & P-08 & (A) & $02 / 18 / 03$ & WAP & I & P03089:08 & Tank condition was normal. \\
\hline $\mathrm{F}$ & 28 & P-09 & (A) & 02/18/03 & WAP & I & P03089:09 & Tank condition was normal. \\
\hline $\mathrm{F}$ & 28 & $P-10$ & (A) & 02/18/03 & WAP & I & P03089:10 & Tank condition was normal. \\
\hline $\mathrm{F}$ & 28 & $\mathrm{P}-11$ & (A) & $02 / 18 / 03$ & WAP & l & P03089:11 & Tank condition was normal. \\
\hline $\mathrm{F}$ & 28 & $P-12$ & (A) & 02/18/03 & WAP & l & P03089:12 & Tank condition was normal. \\
\hline $\mathrm{F}$ & 28 & $P-13$ & (A) & 02/18/03 & WAP & l & P03089:13 & Tank condition was normal. \\
\hline $\mathrm{F}$ & 28 & $\mathrm{P}-14$ & (A) & 02/18/03 & WAP & I & P03089:14 & Tank condition was normal. \\
\hline $\mathrm{H}$ & 29 & $A-01$ & (A) & 01/09/03 & CCTV & I & 907 & $\begin{array}{l}\text { The conductivity probe was } \\
\text { properly deployed per setpoint } \\
\text { document J-JX-G-0001. }\end{array}$ \\
\hline $\mathrm{H}$ & 29 & $A-01$ & (A) & $02 / 04 / 03$ & DP & I & P03080:01-26 & Tank condition was normal. \\
\hline $\mathrm{H}$ & 29 & $\mathrm{~A}-02$ & (A) & 01/06/03 & DP & I & P03005:01-25 & Tank condition was normal. \\
\hline $\mathrm{H}$ & 29 & A-02 & (A) & 01/09/03 & CCTV & I & 907 & $\begin{array}{l}\text { The conductivity probe was } \\
\text { properly deployed per setpoint } \\
\text { document J-JX-G-0001. }\end{array}$ \\
\hline
\end{tabular}




\begin{tabular}{|c|c|c|c|c|c|c|c|c|}
\hline \multirow{2}{*}{$\frac{\text { AREA }}{H}$} & \multirow{2}{*}{$\begin{array}{c}\begin{array}{c}\text { TANK OR } \\
\text { ANCILLARY }\end{array} \\
29\end{array}$} & \multicolumn{2}{|c|}{$\frac{\text { ACCESS OPENING }}{(A \text { OR I) }}$} & \multirow{2}{*}{$\frac{\text { DATE }}{01 / 09 / 03}$} & \multicolumn{3}{|c|}{$\begin{array}{l}\text { INSPECTION METHOD } \\
\text { IDENTIFICATION NUMBER }\end{array}$} & \multirow{2}{*}{$\begin{array}{l}\qquad \text { REMARKS } \\
\qquad \begin{array}{l}\text { The conductivity probe was } \\
\text { properly deployed per setpoint } \\
\text { document J-JX-G-0001. }\end{array}\end{array}$} \\
\hline & & A-03 & (A) & & CCTV & l & 907 & \\
\hline $\mathrm{H}$ & 29 & $\mathrm{~A}-03$ & (A) & $02 / 04 / 03$ & DP & l & P03079:01-27 & Tank condition was normal. \\
\hline $\mathrm{H}$ & 29 & A-04 & (A) & 01/09/03 & CCTV & l & 907 & $\begin{array}{l}\text { The magnetically mounted } \\
\text { thermocouple was deployed per } \\
\text { Structural Integrity Data Sheet } \\
\text { T-DS-G-00010. }\end{array}$ \\
\hline $\mathrm{H}$ & 29 & A-04 & (A) & $02 / 04 / 03$ & DP & l & P03078:01-27 & Tank condition was normal. \\
\hline $\mathrm{H}$ & 29 & P-01 & (A) & $01 / 05 / 03$ & WAP & l & P03027:01 & Tank condition was normal. \\
\hline $\mathrm{H}$ & 29 & P-02 & (A) & $01 / 05 / 03$ & WAP & l & P03027:02 & Tank condition was normal. \\
\hline $\mathrm{H}$ & 29 & P-03 & (A) & $01 / 05 / 03$ & WAP & l & P03027:03 & Tank condition was normal. \\
\hline $\mathrm{H}$ & 29 & $\mathrm{P}-04$ & (A) & $01 / 05 / 03$ & WAP & I & P03027:04 & Tank condition was normal. \\
\hline $\mathrm{H}$ & 29 & P-05 & (A) & $01 / 05 / 03$ & WAP & l & P03027:05 & Tank condition was normal. \\
\hline $\mathrm{H}$ & 29 & P-06 & (A) & $01 / 05 / 03$ & WAP & I & P03027:06 & Tank condition was normal. \\
\hline $\mathrm{H}$ & 29 & P-07 & (A) & $01 / 05 / 03$ & WAP & l & P03027:07 & Tank condition was normal. \\
\hline $\mathrm{H}$ & 29 & P-08 & (A) & $01 / 05 / 03$ & WAP & l & P03027:08 & Tank condition was normal. \\
\hline $\mathrm{H}$ & 29 & P-09 & (A) & $01 / 05 / 03$ & WAP & l & P03027:09 & Tank condition was normal. \\
\hline $\mathrm{H}$ & 29 & P-10 & (A) & $01 / 05 / 03$ & WAP & I & P03027:10 & Tank condition was normal. \\
\hline $\mathrm{H}$ & 29 & $\mathrm{P}-11$ & (A) & $01 / 05 / 03$ & WAP & I & P03027:11 & Tank condition was normal. \\
\hline $\mathrm{H}$ & 29 & $\mathrm{P}-12$ & (A) & $01 / 05 / 03$ & WAP & l & P03027:12 & Tank condition was normal. \\
\hline $\mathrm{H}$ & 29 & $P-13$ & (A) & $01 / 05 / 03$ & WAP & I & P03027:13 & Tank condition was normal. \\
\hline $\mathrm{H}$ & 29 & P-14 & (A) & $01 / 05 / 03$ & WAP & l & P03027:14 & Tank condition was normal. \\
\hline $\mathrm{H}$ & 30 & $A-01$ & (A) & 01/10/03 & CCTV & l & 907 & $\begin{array}{l}\text { The conductivity probe was } \\
\text { properly deployed per setpoint } \\
\text { document J-JX-G-0001. }\end{array}$ \\
\hline $\mathrm{H}$ & 30 & $\mathrm{~A}-01$ & (A) & 01/10/03 & DP & I & P03023:01-26 & Tank condition was normal. \\
\hline
\end{tabular}




\begin{tabular}{|c|c|c|c|c|c|c|c|}
\hline \multirow{2}{*}{$\frac{\text { AREA }}{H}$} & \multirow{2}{*}{$\begin{array}{c}\begin{array}{c}\text { TANK OR } \\
\text { ANCILLARY }\end{array} \\
30\end{array}$} & $\frac{\text { ACCESS OPENING }}{(A \text { OR I) }}$ & \multirow{2}{*}{$\frac{\text { DATE }}{06 / 12 / 03}$} & \multicolumn{3}{|c|}{$\frac{\text { INSPECTION METHOD }}{\text { IDENTIFICATION NUMBER }}$} & \multirow[b]{2}{*}{$\begin{array}{l}\qquad \text { REMARKS } \\
\text { UT scanning was performed on a } \\
\text { vertical strip } 8.5 \text { inches wide for } \\
\text { the entire accessible height of the } \\
\text { primary vessel wall. Analysis of } \\
\text { the data revealed no reportable } \\
\text { cracking, thinning or pitting. This } \\
\text { information is included in } \\
\text { document WSRC-TR-2003-00370 }\end{array}$} \\
\hline & & $A-01 \quad(A)$ & & UT & I & UT-03-006 & \\
\hline
\end{tabular}

$\mathrm{H}$

$\mathrm{H}$

$\mathrm{H}$

$\mathrm{H}$

$\mathrm{H}$

$\mathrm{H}$

$\mathrm{H}$

$\mathrm{H}$

$\mathrm{H}$

$\mathrm{H}$

30

30

30

30

30

30

$\mathrm{H}$

30
A-02 (A) 01/09/03

A-02 (A)

A-03 (A)

01/09/03

A-03 (A) $\quad 01 / 09 / 03$

A-04 (A) $\quad 01 / 10 / 03$

A-04 (A)

01/10/03

P-01 (A)

(A) $\quad 01 / 10 / 03$

P-02 (A)

01/10/03

P-03

(A)

01/10/03

P-04 (A) 01/10/03

P-05

(A)

$10 / 25 / 03$

CCTV / 907

The conductivity probe was properly deployed per setpoint document J-JX-G-0001.

DP I P03024:01-27 Tank condition was normal.

$\begin{array}{lll}\text { CCTV I } 907 & \text { The conductivity probe was }\end{array}$ properly deployed per setpoint document J-JX-G-0001.

DP I P03025:01-27 Tank condition was normal. Stains on the secondary vessel wall and annulus floor were due to the inleakage of water.

$\begin{array}{lll}\text { CCTV } \quad \text { I } 907 & \text { The magnetically mounted }\end{array}$ thermocouple was deployed per Structural Integrity Data Sheet T-DS-G-00010.

\section{DP I P03026:01-27}

Tank condition was normal. Stains on the secondary vessel wall and ventilation duct were due to the inleakage of water.

WAP I P03045:10 Tank condition was normal.

WAP I P03045:11 Tank condition was normal.

WAP I P03045:12 Tank condition was normal.

WAP I P03045:13 Tank condition was normal. Stains and marks on the secondary vessel wall had increased due to the the inleakage of water.

Tank condition was normal. Stains and marks on the secondary vessel wall had increased due to the inleakage of water. 


\begin{tabular}{|c|c|c|c|c|c|c|c|}
\hline \multirow{2}{*}{$\frac{\text { AREA }}{\mathrm{H}}$} & \multirow{2}{*}{$\begin{array}{c}\begin{array}{c}\text { TANK OR } \\
\text { ANCILLARY }\end{array} \\
30\end{array}$} & $\frac{\text { ACCESS OPENING }}{(A \text { OR I) }}$ & \multirow{2}{*}{$\frac{\text { DATE }}{01 / 05 / 03}$} & \multicolumn{3}{|c|}{$\frac{\text { INSPECTION METHOD }}{\text { IDENTIFICATION NUMBER }}$} & \multirow{2}{*}{$\begin{array}{l}\text { REMARKS } \\
\text { Tank condition was normal. } \\
\text { Stains on the secondary vessel } \\
\text { wall were due to the inleakage of } \\
\text { water. }\end{array}$} \\
\hline & & $\mathrm{P}-06 \quad(\mathrm{~A})$ & & WAP & I & P03045:01 & \\
\hline
\end{tabular}

$\mathrm{H}$

30

P-06 (A) 06/13/03

$\begin{array}{lll}\text { UT } \quad / \quad \text { UT-03-007 } & -1\end{array}$
UT scanning was performed on the top knuckle plate and top plate 8.5 inches wide on the primary vessel wall. Analysis of the data revealed no reportable cracking, thinning or pitting. A two square foot area was also scanned on three of the four secondary liner plates. No thinning or pitting was detected. This information is included in document WSRC-TR-2003-00370.

\begin{tabular}{|c|c|c|c|}
\hline WAP & I & P03045:02 & Tank condition was normal. \\
\hline WAP & l & P03045:03 & Tank condition was normal. \\
\hline WAP & । & P03045:07 & Tank condition was normal. \\
\hline WAP & 1 & P03045:08 & Tank condition was normal. \\
\hline WAP & & P03045:09 & Tank condition was normal. \\
\hline WAP & & P03045:04 & Tank condition was normal. \\
\hline WAP & & P03045:05 & Tank condition was normal. \\
\hline WAP & & P03045:06 & Tank condition was norm \\
\hline
\end{tabular}

CCTV I $923 \quad$ Inspection documented salt formations on B2 and B10 cooling coil bundles. The bundles are encrusted with approximately 4 - 5 inches of salt approximately $21 / 2$ feet above the liquid level.

CCTV was used to leak check the transfer jet. No leaks were observed.

Inspection was made to document the salt formations on the cooling coil bundles. The cooling coil bundles had approximately 4 inches of salt accumulated on the coils exposed above the liquid level. 


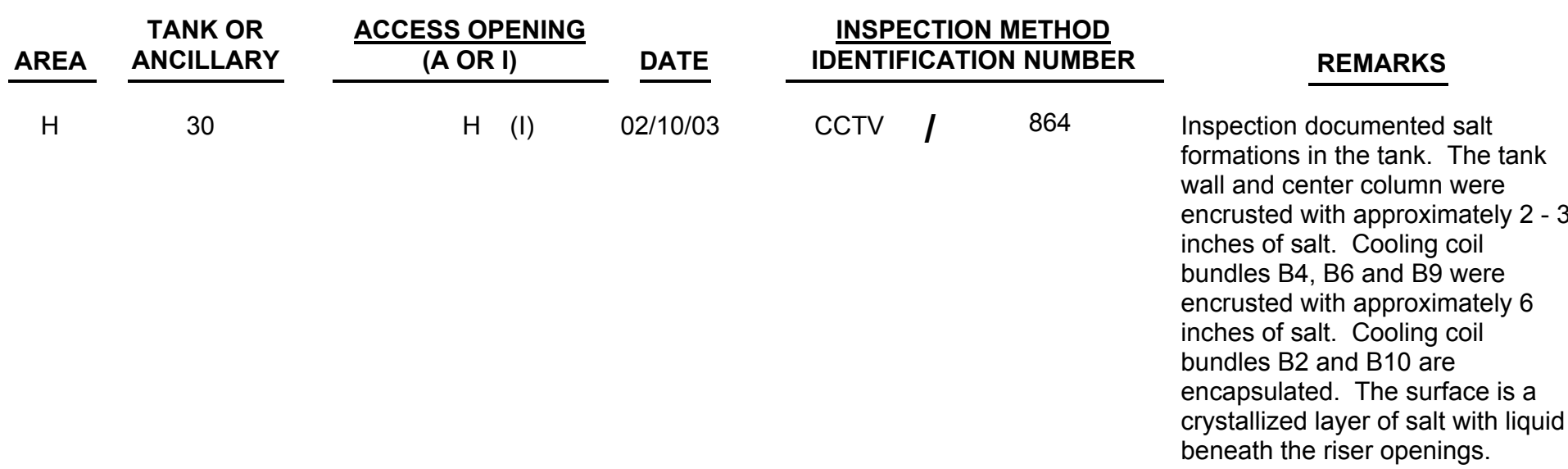

Inspection documented the conditions of the tank interior. The surface of the waste was salt with a pool of liquid beneath the HLLCP and reel tape. Salt formations were observed on the cooling coils and tank wall.

$\mathrm{H}$

$\mathrm{H}$

$\mathrm{H}$

$\mathrm{H}$

$\mathrm{H}$

$\mathrm{H}$

$H$

$\mathrm{H}$

$\mathrm{H}$

$\mathrm{H}$

$\mathrm{H}$

$\mathrm{H}$

\section{$\mathrm{A}-01 \quad$ (A) $\quad 01 / 06 / 03$}

A-01 (A) 01/09/03

A-02 (A) 01/06/03

A-02 (A)

A-03 (A) 01/09/03

A-03

(A)

A-04 (A)

A-04

(A)

31

31

31

31

31
P-01

(A)

01/05/03

$\mathrm{P}-02$

(A)

01/05/03

P-03

(A)

01/05/03

P-04 (A) 01/05/03
01/09/03

01/09/03

$\mathrm{DP} \quad / \quad \mathrm{P} 03007: 01-25$

CCTV / $\quad 907$

DP

CCTV

907

01/06/03

01/09/03

C




\begin{tabular}{|c|c|c|c|c|c|c|c|c|}
\hline \multirow{2}{*}{$\frac{\text { AREA }}{H}$} & \multirow{2}{*}{$\begin{array}{c}\begin{array}{c}\text { TANK OR } \\
\text { ANCILLARY }\end{array} \\
31\end{array}$} & \multicolumn{2}{|c|}{$\frac{\text { ACCESS OPENING }}{(\text { A OR I) }}$} & \multirow{2}{*}{$\frac{\text { DATE }}{01 / 05 / 03}$} & \multicolumn{3}{|c|}{$\frac{\text { INSPECTION METHOD }}{\text { IDENTIFICATION NUMBER }}$} & \multirow{2}{*}{$\frac{\text { REMARKS }}{\text { Tank condition was normal. }}$} \\
\hline & & $\mathrm{P}-05$ & (A) & & WAP & l & P03022:05 & \\
\hline $\mathrm{H}$ & 31 & P-06 & (A) & $01 / 05 / 03$ & WAP & l & P03022:06 & Tank condition was normal. \\
\hline $\mathrm{H}$ & 31 & P-06 & (A) & 06/03/03 & UT & l & UT-03-005 & $\begin{array}{l}\text { UT scanning was performed on a } \\
\text { vertical strip } 8.5 \text { inches wide for } \\
\text { the entire accessible height of the } \\
\text { primary vessel wall. One incipient } \\
\text { pit was detected four inches } \\
\text { above the top knuckle weld. The } \\
\text { pit was } 0.046 \text { inches deep with a } \\
\text { remaining ligament (minimum } \\
\text { thickness) of } 0.443 \text { inches. } \\
\text { Analysis of the data revealed no } \\
\text { reportable cracking, thinning or } \\
\text { pitting. A two square foot area } \\
\text { was also scanned on three of the } \\
\text { four secondary liner plates for } \\
\text { thinning and pitting. This } \\
\text { information is included in } \\
\text { document WSRC-TR-2003-00370. }\end{array}$ \\
\hline $\mathrm{H}$ & 31 & P-07 & (A) & $01 / 05 / 03$ & WAP & l & P03022:07 & Tank condition was normal. \\
\hline $\mathrm{H}$ & 31 & P-08 & (A) & $01 / 05 / 03$ & WAP & l & P03022:08 & Tank condition was normal. \\
\hline $\mathrm{H}$ & 31 & P-09 & (A) & $01 / 05 / 03$ & WAP & l & P03022:09 & Tank condition was normal. \\
\hline $\mathrm{H}$ & 31 & $\mathrm{P}-10$ & (A) & $01 / 05 / 03$ & WAP & l & P03022:10 & Tank condition was normal. \\
\hline $\mathrm{H}$ & 31 & $\mathrm{P}-11$ & (A) & $01 / 05 / 03$ & WAP & l & P03022:11 & Tank condition was normal. \\
\hline $\mathrm{H}$ & 31 & $\mathrm{P}-12$ & (A) & $01 / 05 / 03$ & WAP & l & P03022:12 & Tank condition was normal. \\
\hline $\mathrm{H}$ & 31 & $\mathrm{P}-13$ & (A) & 01/05/03 & WAP & l & P03022:13 & Tank condition was normal. \\
\hline $\mathrm{H}$ & 31 & $\mathrm{P}-14$ & (A) & $01 / 05 / 03$ & WAP & l & P03022:14 & Tank condition was normal. \\
\hline $\mathrm{H}$ & 32 & $\mathrm{~A}-01$ & (A) & 01/06/03 & DP & l & P03001:01-25 & Tank condition was normal. \\
\hline $\mathrm{H}$ & 32 & $\mathrm{~A}-01$ & (A) & 01/09/03 & CCTV & l & 907 & $\begin{array}{l}\text { The conductivity probe was } \\
\text { properly deployed per setpoint } \\
\text { document J-JX-G-0001. }\end{array}$ \\
\hline $\mathrm{H}$ & 32 & $\mathrm{~A}-02$ & (A) & 01/06/03 & DP & I & P03002:01-25 & Tank condition was normal. \\
\hline $\mathrm{H}$ & 32 & $\mathrm{~A}-02$ & (A) & $01 / 09 / 03$ & CCTV & l & 907 & $\begin{array}{l}\text { The conductivity probe was } \\
\text { properly deployed per setpoint } \\
\text { document J-JX-G-0001. }\end{array}$ \\
\hline $\mathrm{H}$ & 32 & $\mathrm{~A}-03$ & (A) & 01/06/03 & DP & I & P03003:01-28 & Tank condition was normal. \\
\hline
\end{tabular}




\begin{tabular}{|c|c|c|c|c|c|c|c|}
\hline \multirow{2}{*}{$\frac{\text { AREA }}{\mathrm{H}}$} & \multirow{2}{*}{$\begin{array}{c}\begin{array}{c}\text { TANK OR } \\
\text { ANCILLARY }\end{array} \\
32\end{array}$} & $\frac{\text { ACCESS OPENING }}{(A \text { OR I) }}$ & \multirow{2}{*}{$\frac{\text { DATE }}{01 / 09 / 03}$} & \multicolumn{3}{|c|}{$\begin{array}{l}\text { INSPECTION METHOD } \\
\text { IDENTIFICATION NUMBER }\end{array}$} & \multirow{2}{*}{$\begin{array}{l}\qquad \text { REMARKS } \\
\qquad \begin{array}{l}\text { The conductivity probe was } \\
\text { properly deployed per setpoint } \\
\text { document J-JX-G-0001. }\end{array}\end{array}$} \\
\hline & & $\mathrm{A}-03 \quad(\mathrm{~A})$ & & CCTV & I & 907 & \\
\hline $\mathrm{H}$ & 32 & $\mathrm{~A}-04 \quad(\mathrm{~A})$ & $01 / 06 / 03$ & DP & l & P03004:01-27 & Tank condition was normal. \\
\hline $\mathrm{H}$ & 32 & $\mathrm{~A}-04 \quad(\mathrm{~A})$ & 01/09/03 & CCTV & l & 907 & $\begin{array}{l}\text { The magnetically mounted } \\
\text { thermocouple was deployed per } \\
\text { Structural Integrity Data Sheet } \\
\text { T-DS-G-00010. }\end{array}$ \\
\hline
\end{tabular}

$\mathrm{H}$

01/05/03

WAP / P03039:01

Tank condition was normal.

UT scanning was performed on a vertical strip 8.5 inches wide for the entire accessible height of the primary vessel wall. Analysis of the data revealed no reportable cracking, thinning or pitting. A weld attachment was also examined for cracking. No crack-like indications were detected. This information is included in document WSRC-TR-2003-00370.

Tank condition was normal.

Tank condition was normal.

Tank condition was normal. Stains and marks on the secondary vessel wall had increased due to the inleakage of water.

UT scanning was performed on a vertical strip 8.5 inches wide for the entire accessible height of the primary vessel wall. Analysis of the data revealed no reportable cracking, thinning or pitting. Approximately 13 feet of the top weld of the bottom knuckle plate and the weld between the middle and lower plates was examined for cracking parallel and perpendicular to the weld. No cracking was detected. Five weld repairs were examined on the horizontal welds and no crack-like indications were detected. This information is included in document WSRC-TR-2003-00370. 


\begin{tabular}{|c|c|c|c|c|c|c|c|c|}
\hline \multirow{2}{*}{$\frac{\text { AREA }}{H}$} & \multirow{2}{*}{$\begin{array}{c}\text { TANK OR } \\
\text { ANCILLARY } \\
32\end{array}$} & \multicolumn{2}{|c|}{$\frac{\text { ACCESS OPENING }}{(A \text { OR I) }}$} & \multirow{2}{*}{$\frac{\text { DATE }}{01 / 05 / 03}$} & \multicolumn{3}{|c|}{$\begin{array}{l}\text { INSPECTION METHOD } \\
\text { IDENTIFICATION NUMBER }\end{array}$} & \multirow{2}{*}{$\frac{\text { REMARKS }}{\text { Tank condition was normal. }}$} \\
\hline & & P-06 & (A) & & WAP & I & P03039:06 & \\
\hline $\mathrm{H}$ & 32 & P-07 & (A) & 01/05/03 & WAP & l & P03039:07 & Tank condition was normal. \\
\hline $\mathrm{H}$ & 32 & P-07 & (A) & $04 / 24 / 03$ & UT & l & UT-03-003 & $\begin{array}{l}\text { UT scanning was performed on a } \\
\text { vertical strip } 8.5 \text { inches wide for } \\
\text { the entire accessible height of the } \\
\text { primary vessel wall. Analysis of } \\
\text { the data revealed no reportable } \\
\text { cracking, thinning or pitting. A top } \\
\text { plate vertical weld near P-8 was } \\
\text { examined for cracking parallel and } \\
\text { perpendicular to the weld. No } \\
\text { cracking was detected. This } \\
\text { information is included in } \\
\text { document WSRC-TR-2003-00370. }\end{array}$ \\
\hline $\mathrm{H}$ & 32 & $\mathrm{P}-08$ & (A) & $01 / 05 / 03$ & WAP & l & P03039:08 & Tank condition was normal. \\
\hline $\mathrm{H}$ & 32 & P-09 & (A) & $01 / 05 / 03$ & WAP & l & P03039:09 & Tank condition was normal. \\
\hline $\mathrm{H}$ & 32 & P-10 & (A) & $01 / 05 / 03$ & WAP & l & P03039:10 & Tank condition was normal. \\
\hline $\mathrm{H}$ & 32 & $\mathrm{P}-11$ & (A) & $01 / 05 / 03$ & WAP & l & P03039:11 & Tank condition was normal. \\
\hline $\mathrm{H}$ & 32 & $\mathrm{P}-12$ & (A) & $01 / 05 / 03$ & WAP & l & P03039:13 & Tank condition was normal. \\
\hline $\mathrm{H}$ & 32 & $P-13$ & (A) & $01 / 05 / 03$ & WAP & l & P03039:14 & Tank condition was normal. \\
\hline $\mathrm{H}$ & 32 & P-14 & (A) & 01/05/03 & WAP & l & P03039:12 & Tank condition was normal. \\
\hline $\mathrm{H}$ & 32 & P-14 & (A) & 03/14/03 & UT & l & UT-03-001 & $\begin{array}{l}\text { UT scanning was performed on a } \\
\text { vertical strip } 8.5 \text { inches wide for } \\
\text { the entire accessible height of the } \\
\text { primary vessel wall. Analysis of } \\
\text { the data revealed no reportable } \\
\text { cracking, thinning or pitting. This } \\
\text { information is included in } \\
\text { document WSRC-TR-2003-00370. }\end{array}$ \\
\hline $\mathrm{H}$ & 32 & P-15 & (A) & 07/30/03 & WAP & l & P03154:01 & Tank condition was normal. \\
\hline $\mathrm{H}$ & 32 & $\mathrm{H}$ & (I) & $01 / 21 / 03$ & CCTV & l & 876 & $\begin{array}{l}\text { Inspection of cooling coil bundles } \\
\text { revealed that the bundles were } \\
\text { encrusted with approximately } 6 \text { - } 8 \\
\text { inches of salt. Salt formations } \\
\text { were observed on the tank wall. }\end{array}$ \\
\hline
\end{tabular}




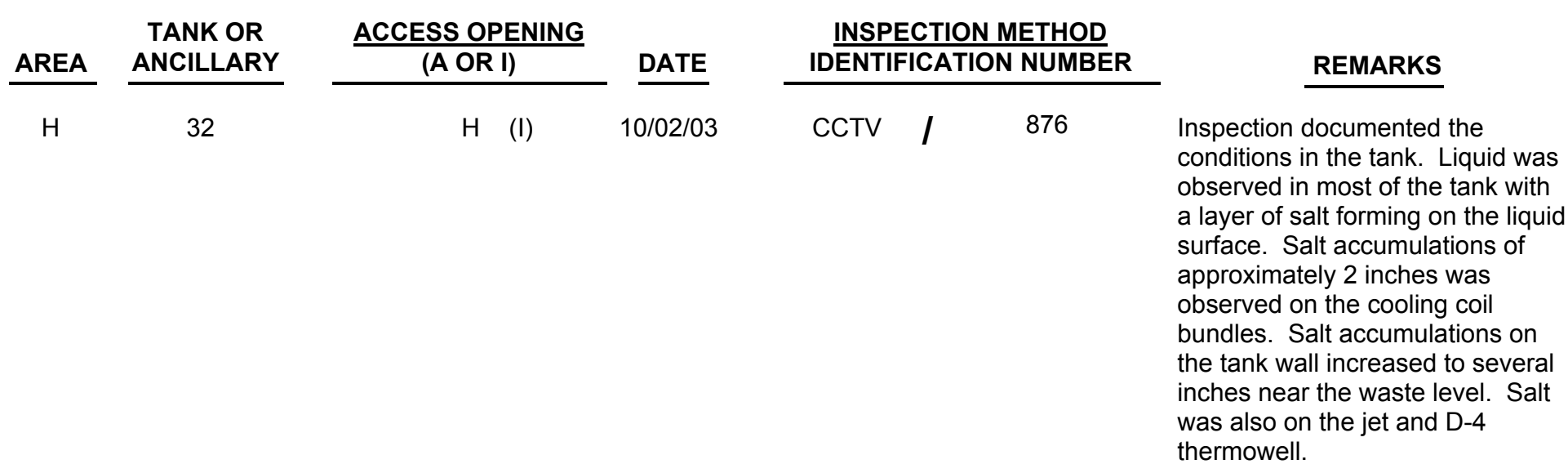

$$
\text { HELIUM / }
$$

03/27/03

04/02/03

A-01
(A)

07/08/03

$\mathrm{A}-01 \quad(\mathrm{~A})$

07/11/03

A-02

(A)

07/08/03

A-02 (A)

07/11/03

CCTV

926
HELIUM / HE-03-005
HE-03-001

Inspection was made to document salt accumulation in the tank. No salt formations were observed on any exposed surfaces.

Helium tracer test of the transfer line from FDB-03 to Tank 33 identified leak site locations for excavation and repair.

A helium tracer test was performed to verify the integrity of the FDB-03 to Tank 33 transfer line after repairs. Additional leak sites were identified for further excavation and repair.

A helium tracer test was performed to verify the integrity of the FDB-03 to Tank 33 transfer line after repairs. Additional leak sites were identified for further excavation and repair.

A helium tracer test verified the integrity of the FDB-03 to Tank 33 transfer line.

Tank condition was normal.

The conductivity probe was properly deployed per setpoint document J-JX-G-0001.

Tank condition was normal.

The conductivity probe was properly deployed per setpoint document J-JX-G-0001.

DP / P03141:01-27 Tank condition was normal. 


\begin{tabular}{|c|c|c|c|c|c|c|c|c|}
\hline \multirow{2}{*}{$\frac{\text { AREA }}{F}$} & \multirow{2}{*}{$\begin{array}{c}\begin{array}{c}\text { TANK OR } \\
\text { ANCILLARY }\end{array} \\
33\end{array}$} & \multicolumn{2}{|c|}{$\frac{\text { ACCESS OPENING }}{(A \text { OR I) }}$} & \multirow{2}{*}{$\frac{\text { DATE }}{07 / 11 / 03}$} & \multicolumn{3}{|c|}{$\frac{\text { INSPECTION METHOD }}{\text { IDENTIFICATION NUMBER }}$} & \multirow{2}{*}{$\begin{array}{l}\qquad \text { REMARKS } \\
\text { The conductivity probe was } \\
\text { properly deployed per setpoint } \\
\text { document J-JX-G-0001. }\end{array}$} \\
\hline & & A-03 & (A) & & CCTV & l & 926 & \\
\hline $\mathrm{F}$ & 33 & A-04 & (A) & 07/08/03 & DP & l & P03142:01-27 & Tank condition was normal. \\
\hline $\mathrm{F}$ & 33 & A-04 & (A) & $07 / 11 / 03$ & CCTV & l & 926 & $\begin{array}{l}\text { The magnetically mounted } \\
\text { thermocouple was deployed per } \\
\text { Structural Integrity Data Sheet } \\
\text { T-DS-G-00010. }\end{array}$ \\
\hline $\mathrm{F}$ & 33 & P-01 & (A) & 07/09/03 & WAP & l & P03150:01 & Tank condition was normal. \\
\hline $\mathrm{F}$ & 33 & P-02 & (A) & 07/09/03 & WAP & l & P03150:02 & Tank condition was normal. \\
\hline $\mathrm{F}$ & 33 & P-03 & (A) & 07/09/03 & WAP & l & P03150:03 & Tank condition was normal. \\
\hline $\mathrm{F}$ & 33 & P-04 & (A) & 07/09/03 & WAP & l & P03150:04 & Tank condition was normal. \\
\hline $\mathrm{F}$ & 33 & P-05 & (A) & 07/09/03 & WAP & l & P03150:05 & Tank condition was normal. \\
\hline $\mathrm{F}$ & 33 & P-06 & (A) & 07/09/03 & WAP & l & P03150:06 & Tank condition was normal. \\
\hline $\mathrm{F}$ & 33 & P-07 & (A) & 07/09/03 & WAP & I & P03150:07 & Tank condition was normal. \\
\hline $\mathrm{F}$ & 33 & P-08 & (A) & 07/09/03 & WAP & l & P03150:08 & Tank condition was normal. \\
\hline $\mathrm{F}$ & 33 & P-09 & (A) & 07/09/03 & WAP & l & P03150:09 & Tank condition was normal. \\
\hline $\mathrm{F}$ & 33 & $P-10$ & (A) & 07/09/03 & WAP & l & P03150:10 & Tank condition was normal. \\
\hline $\mathrm{F}$ & 33 & $P-11$ & (A) & 07/09/03 & WAP & l & P03150:11 & Tank condition was normal. \\
\hline $\mathrm{F}$ & 33 & $\mathrm{P}-12$ & (A) & 07/09/03 & WAP & l & P03150:12 & Tank condition was normal. \\
\hline $\mathrm{F}$ & 33 & $P-13$ & (A) & 07/09/03 & WAP & l & P03150:13 & Tank condition was normal. \\
\hline $\mathrm{F}$ & 33 & P-14 & (A) & 07/09/03 & WAP & l & P03150:14 & Tank condition was normal. \\
\hline $\mathrm{F}$ & 33 & P-15 & (A) & 07/09/03 & WAP & I & P03150:15 & Tank condition was normal. \\
\hline $\mathrm{F}$ & 33 & P-16 & (A) & 07/09/03 & WAP & l & P03150:16 & Tank condition was normal. \\
\hline $\mathrm{F}$ & 34 & & & 03/19/03 & HELIUM & l & HE-03-002 & $\begin{array}{l}\text { A helium tracer test verified the } \\
\text { integrity of the FDB-03 to Tank } 34 \\
\text { transfer line. }\end{array}$ \\
\hline $\mathrm{F}$ & 34 & $A-01$ & (A) & 07/08/03 & DP & l & P03135:01-27 & Tank condition was normal. \\
\hline
\end{tabular}




\begin{tabular}{|c|c|c|c|c|c|c|c|}
\hline \multirow{2}{*}{$\frac{\text { AREA }}{F}$} & \multirow{2}{*}{$\begin{array}{c}\begin{array}{c}\text { TANK OR } \\
\text { ANCILLARY }\end{array} \\
34\end{array}$} & $\frac{\text { ACCESS OPENING }}{(\text { A OR I) }}$ & \multirow{2}{*}{$\frac{\text { DATE }}{07 / 11 / 03}$} & \multicolumn{3}{|c|}{$\begin{array}{l}\text { INSPECTION METHOD } \\
\text { IDENTIFICATION NUMBER }\end{array}$} & \multirow{2}{*}{$\begin{array}{l}\qquad \text { REMARKS } \\
\qquad \begin{array}{l}\text { The conductivity probe was } \\
\text { properly deployed per setpoint } \\
\text { document J-JX-G-0001. }\end{array}\end{array}$} \\
\hline & & $A-01 \quad(A)$ & & CCTV & l & 926 & \\
\hline $\mathrm{F}$ & 34 & $\mathrm{~A}-02 \quad(\mathrm{~A})$ & 07/08/03 & DP & l & P03136:01-27 & Tank condition was normal. \\
\hline $\mathrm{F}$ & 34 & $\mathrm{~A}-02 \quad(\mathrm{~A})$ & $07 / 11 / 03$ & CCTV & l & 926 & $\begin{array}{l}\text { The conductivity probe was } \\
\text { properly deployed per setpoint } \\
\text { document J-JX-G-0001. }\end{array}$ \\
\hline $\mathrm{F}$ & 34 & $\mathrm{~A}-03 \quad(\mathrm{~A})$ & 07/08/03 & DP & l & P03137:01-28 & Tank condition was normal. \\
\hline $\mathrm{F}$ & 34 & $\mathrm{~A}-03 \quad(\mathrm{~A})$ & $07 / 11 / 03$ & CCTV & l & 926 & $\begin{array}{l}\text { The conductivity probe was } \\
\text { properly deployed per setpoint } \\
\text { document J-JX-G-0001. }\end{array}$ \\
\hline $\mathrm{F}$ & 34 & $\mathrm{~A}-04 \quad(\mathrm{~A})$ & 07/08/03 & DP & l & P03138:01-27 & $\begin{array}{l}\text { Tank condition was normal. } \\
\text { However, a dark stain originating } \\
\text { near the upper plate weld was } \\
\text { observed. Further investigations } \\
\text { will be performed. }\end{array}$ \\
\hline $\mathrm{F}$ & 34 & $\mathrm{~A}-04 \quad(\mathrm{~A})$ & 07/11/03 & CCTV & I & 926 & $\begin{array}{l}\text { The magnetically mounted } \\
\text { thermocouple was deployed per } \\
\text { Structural Integrity Data Sheet } \\
\text { T-DS-G-00010. }\end{array}$ \\
\hline
\end{tabular}

Inspection of a stain originating near the upper plate weld towards riser $\mathrm{P}-03$ was inconclusive. Further investigations will be performed.

CCTV facilitated remote operations during swiping of a suspect area (stain) on the tank wall. No contamination was detected indicating a surface marking and not a crack.

A stain observed on a routine visual inspection was examined. Analysis of the data revealed no reportable cracking, thinning or pitting. This information is included in document WSRC-TR-2003-00370.

F

Tank condition was normal.

Tank condition was normal.
P-01
(A) $\quad 07 / 27 / 03$

WAP / P03153:01

Tank condition was normal. 


\begin{tabular}{|c|c|c|c|c|c|c|c|c|}
\hline \multirow{2}{*}{$\frac{\text { AREA }}{F}$} & \multirow{2}{*}{$\begin{array}{c}\begin{array}{c}\text { TANK OR } \\
\text { ANCILLARY }\end{array} \\
34\end{array}$} & \multicolumn{2}{|c|}{$\frac{\text { ACCESS OPENING }}{(A \text { OR I) }}$} & \multirow{2}{*}{$\frac{\text { DATE }}{07 / 27 / 03}$} & \multicolumn{3}{|c|}{$\frac{\text { INSPECTION METHOD }}{\text { IDENTIFICATION NUMBER }}$} & \multirow{2}{*}{$\frac{\text { REMARKS }}{\text { Tank condition was normal. }}$} \\
\hline & & P-03 & (A) & & WAP & l & P03153:03 & \\
\hline $\mathrm{F}$ & 34 & $\mathrm{P}-04$ & (A) & $07 / 27 / 03$ & WAP & l & P03153:04 & Tank condition was normal. \\
\hline $\mathrm{F}$ & 34 & P-05 & (A) & $07 / 27 / 03$ & WAP & l & P03153:05 & Tank condition was normal. \\
\hline $\mathrm{F}$ & 34 & P-06 & (A) & $07 / 27 / 03$ & WAP & l & P03153:06 & Tank condition was normal. \\
\hline $\mathrm{F}$ & 34 & P-07 & (A) & $07 / 27 / 03$ & WAP & l & P03153:07 & Tank condition was normal. \\
\hline $\mathrm{F}$ & 34 & $\mathrm{P}-08$ & (A) & $07 / 27 / 03$ & WAP & l & P03153:08 & Tank condition was normal. \\
\hline $\mathrm{F}$ & 34 & P-09 & (A) & $06 / 26 / 03$ & UT & l & UT-03-008 & $\begin{array}{l}\text { UT scanning was performed on a } \\
\text { vertical strip } 8.5 \text { inches wide for } \\
\text { the entire accessible height of the } \\
\text { primary vessel wall. A two square } \\
\text { foot area was also scanned on } \\
\text { three of the four secondary liner } \\
\text { plates. Analysis of the data } \\
\text { revealed no reportable cracking, } \\
\text { thinning, or pitting. This } \\
\text { information is included in } \\
\text { document WSRC-TR-2003-00370 }\end{array}$ \\
\hline $\mathrm{F}$ & 34 & P-09 & (A) & $07 / 27 / 03$ & WAP & l & P03153:09 & Tank condition was normal. \\
\hline $\mathrm{F}$ & 34 & $P-10$ & (A) & $07 / 27 / 03$ & WAP & I & P03153:10 & Tank condition was normal. \\
\hline $\mathrm{F}$ & 34 & $\mathrm{P}-11$ & (A) & $07 / 27 / 03$ & WAP & I & P03153:11 & Tank condition was normal. \\
\hline $\mathrm{F}$ & 34 & $\mathrm{P}-12$ & (A) & $07 / 27 / 03$ & WAP & l & P03153:12 & Tank condition was normal. \\
\hline $\mathrm{F}$ & 34 & $P-13$ & (A) & $07 / 27 / 03$ & WAP & l & $P 03153: 13$ & Tank condition was normal. \\
\hline $\mathrm{F}$ & 34 & $\mathrm{P}-14$ & (A) & $07 / 27 / 03$ & WAP & l & P03153:14 & Tank condition was normal. \\
\hline $\mathrm{F}$ & 34 & $\mathrm{P}-15$ & (A) & $07 / 27 / 03$ & WAP & l & P03153:15 & Tank condition was normal. \\
\hline $\mathrm{F}$ & 34 & $P-16$ & (A) & $07 / 27 / 03$ & WAP & l & P03153:16 & Tank condition was normal. \\
\hline $\mathrm{F}$ & 34 & $\mathrm{H}$ & (I) & $09 / 23 / 03$ & CCTV & l & 849 & $\begin{array}{l}\text { The HLLCP was properly } \\
\text { deployed, and no obstructions } \\
\text { were observed. }\end{array}$ \\
\hline $\mathrm{H}$ & 35 & $\mathrm{~A}-01$ & (A) & $01 / 02 / 03$ & WAP & I & P03046:04 & Tank condition was normal. \\
\hline $\mathrm{H}$ & 35 & $\mathrm{~A}-02$ & (A) & $01 / 02 / 03$ & WAP & I & P03046:02 & Tank condition was normal. \\
\hline $\mathrm{H}$ & 35 & A-02 & (A) & $01 / 08 / 03$ & CCTV & I & 907 & $\begin{array}{l}\text { The conductivity probe was } \\
\text { properly deployed per setpoint } \\
\text { document J-JX-G-0001. }\end{array}$ \\
\hline
\end{tabular}




\begin{tabular}{|c|c|c|c|c|c|c|c|c|}
\hline \multirow{2}{*}{$\frac{\text { AREA }}{\mathrm{H}}$} & \multirow{2}{*}{$\begin{array}{c}\begin{array}{c}\text { TANK OR } \\
\text { ANCILLARY }\end{array} \\
35\end{array}$} & \multicolumn{2}{|c|}{$\frac{\text { ACCESS OPENING }}{(A \text { OR I) }}$} & \multirow{2}{*}{$\frac{\text { DATE }}{01 / 02 / 03}$} & \multicolumn{3}{|c|}{$\frac{\text { INSPECTION METHOD }}{\text { IDENTIFICATION NUMBER }}$} & \multirow{2}{*}{$\frac{\text { REMARKS }}{\text { Tank condition was normal. }}$} \\
\hline & & $\mathrm{A}-03$ & (A) & & WAP & l & P03046:07 & \\
\hline $\mathrm{H}$ & 35 & A-03 & (A) & $01 / 08 / 03$ & CCTV & l & 907 & $\begin{array}{l}\text { The conductivity probe was } \\
\text { properly deployed per setpoint } \\
\text { document J-JX-G-0001. }\end{array}$ \\
\hline $\mathrm{H}$ & 35 & A-03 & (A) & $05 / 06 / 03$ & CCTV & l & 957 & $\begin{array}{l}\text { Inspection verified liquid on the } \\
\text { annulus floor after a conductivity } \\
\text { probe alarm was received. Steam } \\
\text { was blowing into the annulus due } \\
\text { to a leak at the preheater, } \\
\text { condensing on the walls and } \\
\text { collecting on the annulus floor } \\
\text { causing the alarm. }\end{array}$ \\
\hline $\mathrm{H}$ & 35 & A-04 & (A) & $01 / 02 / 03$ & WAP & l & P03046:13 & Tank condition was normal. \\
\hline $\mathrm{H}$ & 35 & A-04 & (A) & $01 / 08 / 03$ & CCTV & l & 907 & $\begin{array}{l}\text { The conductivity probe was } \\
\text { properly deployed per setpoint } \\
\text { document J-JX-G-0001. }\end{array}$ \\
\hline $\mathrm{H}$ & 35 & $\mathrm{P}-01$ & (A) & $01 / 08 / 03$ & DP & l & P03013:01-25 & Tank condition was normal. \\
\hline $\mathrm{H}$ & 35 & P-02 & (A) & $01 / 08 / 03$ & DP & l & P03014:01-25 & $\begin{array}{l}\text { Tank condition was normal. } \\
\text { Stains and marks on the } \\
\text { secondary vessel wall were } \\
\text { caused by the inleakage of water. }\end{array}$ \\
\hline $\mathrm{H}$ & 35 & $\mathrm{P}-03$ & (A) & $01 / 08 / 03$ & DP & l & P03015:01-25 & Tank condition was normal. \\
\hline $\mathrm{H}$ & 35 & P-04 & (A) & $01 / 08 / 03$ & DP & l & P03016:01-26 & $\begin{array}{l}\text { Tank condition was normal. } \\
\text { Stains and marks on the } \\
\text { secondary vessel wall were } \\
\text { caused by the the inleakage of } \\
\text { water. }\end{array}$ \\
\hline $\mathrm{H}$ & 35 & P-05 & (A) & $01 / 02 / 03$ & WAP & l & P03046:03 & Tank condition was normal. \\
\hline $\mathrm{H}$ & 35 & P-05 & (A) & $05 / 06 / 03$ & CCTV & l & 957 & $\begin{array}{l}\text { Inspection verified liquid on the } \\
\text { annulus floor after a conductivity } \\
\text { probe alarm was received. Steam } \\
\text { was blowing into the annulus due } \\
\text { to a leak at the preheater, } \\
\text { condensing on the walls and } \\
\text { collecting on the annulus floor } \\
\text { causing the alarm. }\end{array}$ \\
\hline $\mathrm{H}$ & 35 & P-06 & (A) & $01 / 02 / 03$ & WAP & l & P03046:09 & Tank condition was normal. \\
\hline $\mathrm{H}$ & 35 & P-07 & (A) & $01 / 02 / 03$ & WAP & I & P03046:01 & Tank condition was normal. \\
\hline
\end{tabular}




\begin{tabular}{|c|c|c|c|c|}
\hline AREA & $\begin{array}{l}\text { TANK OR } \\
\text { ANCILLARY }\end{array}$ & $\frac{\text { ACCESS OPENING }}{(A \text { OR I) }}$ & DATE & $\frac{\text { INSPECTION METHOD }}{\text { IDENTIFICATION NUMBER }}$ \\
\hline $\mathrm{H}$ & 35 & $\mathrm{P}-07 \quad(\mathrm{~A})$ & $05 / 06 / 03$ & CCTV \\
\hline
\end{tabular}

\section{REMARKS}

Inspection verified liquid on the annulus floor after a conductivity probe alarm was received. Steam was blowing into the annulus due to a leak at the preheater, condensing on the walls and collecting on the annulus floor causing the alarm.

\begin{tabular}{|c|c|c|c|c|c|c|c|c|}
\hline $\mathrm{H}$ & 35 & P-08 & (A) & $01 / 02 / 03$ & WAP & I & P03046:08 & Tank condition was normal. \\
\hline $\mathrm{H}$ & 35 & P-09 & (A) & $01 / 02 / 03$ & WAP & I & P03046:06 & Tank condition was normal. \\
\hline $\mathrm{H}$ & 35 & P-10 & (A) & $01 / 02 / 03$ & WAP & I & P03046:05 & Tank condition was normal. \\
\hline $\mathrm{H}$ & 35 & $\mathrm{P}-11$ & (A) & $01 / 02 / 03$ & WAP & l & P03046:14 & Tank condition was normal. \\
\hline $\mathrm{H}$ & 35 & $\mathrm{P}-12$ & (A) & $01 / 02 / 03$ & WAP & l & P03046:12 & Tank condition was normal. \\
\hline $\mathrm{H}$ & 35 & $\mathrm{P}-13$ & (A) & $01 / 02 / 03$ & WAP & l & P03046:11 & Tank condition was normal. \\
\hline $\mathrm{H}$ & 35 & $\mathrm{P}-14$ & (A) & $01 / 02 / 03$ & WAP & l & P03046:10 & Tank condition was normal. \\
\hline $\mathrm{H}$ & 35 & $P-14$ & (A) & $05 / 06 / 03$ & CCTV & l & 957 & $\begin{array}{l}\text { Inspection verified liquid on the } \\
\text { annulus floor after a conductivity } \\
\text { probe alarm was received. Steam } \\
\text { was blowing into the annulus due } \\
\text { to a leak at the preheater, } \\
\text { condensing on the walls and } \\
\text { collecting on the annulus floor } \\
\text { causing the alarm. }\end{array}$ \\
\hline $\mathrm{H}$ & 36 & A-01 & (A) & $01 / 02 / 03$ & WAP & l & P03041:14 & Tank condition was normal. \\
\hline $\mathrm{H}$ & 36 & A-02 & (A) & $01 / 02 / 03$ & WAP & l & P03041:02 & Tank condition was normal. \\
\hline $\mathrm{H}$ & 36 & A-02 & (A) & $01 / 07 / 03$ & CCTV & l & 907 & $\begin{array}{l}\text { The conductivity probe was } \\
\text { properly deployed per setpoint } \\
\text { document J-JX-G-0001. }\end{array}$ \\
\hline $\mathrm{H}$ & 36 & A-03 & (A) & $01 / 02 / 03$ & WAP & l & P03041:06 & Tank condition was normal. \\
\hline $\mathrm{H}$ & 36 & A-03 & (A) & $01 / 07 / 03$ & CCTV & I & 907 & $\begin{array}{l}\text { The conductivity probe was } \\
\text { properly deployed per setpoint } \\
\text { document J-JX-G-0001. }\end{array}$ \\
\hline $\mathrm{H}$ & 36 & A-04 & (A) & $01 / 02 / 03$ & WAP & I & P03041:11 & Tank condition was normal. \\
\hline $\mathrm{H}$ & 36 & A-04 & (A) & $01 / 07 / 03$ & CCTV & I & 907 & $\begin{array}{l}\text { The conductivity probe was } \\
\text { properly deployed per setpoint } \\
\text { document J-JX-G-0001. }\end{array}$ \\
\hline
\end{tabular}




\begin{tabular}{|c|c|c|c|c|c|c|c|c|}
\hline \multirow{2}{*}{$\frac{\text { AREA }}{H}$} & \multirow{2}{*}{$\begin{array}{c}\begin{array}{c}\text { TANK OR } \\
\text { ANCILLARY }\end{array} \\
36\end{array}$} & \multicolumn{2}{|c|}{$\frac{\text { ACCESS OPENING }}{(A \text { OR I) }}$} & \multirow{2}{*}{$\frac{\text { DATE }}{01 / 07 / 03}$} & \multicolumn{3}{|c|}{$\begin{array}{l}\text { INSPECTION METHOD } \\
\text { IDENTIFICATION NUMBER }\end{array}$} & \multirow{2}{*}{$\frac{\text { REMARKS }}{\text { Tank condition was normal. }}$} \\
\hline & & P-01 & (A) & & DP & l & P03017:01-27 & \\
\hline $\mathrm{H}$ & 36 & P-02 & (A) & 01/07/03 & DP & l & P03018:01-27 & Tank condition was normal. \\
\hline $\mathrm{H}$ & 36 & P-03 & (A) & 01/07/03 & DP & l & P03019:01-27 & Tank condition was normal. \\
\hline $\mathrm{H}$ & 36 & P-04 & (A) & 01/07/03 & DP & l & P03020:01-27 & Tank condition was normal. \\
\hline $\mathrm{H}$ & 36 & P-05 & (A) & $01 / 02 / 03$ & WAP & l & P03041:01 & Tank condition was normal. \\
\hline $\mathrm{H}$ & 36 & P-06 & (A) & $01 / 02 / 03$ & WAP & l & P03041:03 & Tank condition was normal. \\
\hline $\mathrm{H}$ & 36 & P-07 & (A) & $01 / 02 / 03$ & WAP & l & P03041:04 & Tank condition was normal. \\
\hline $\mathrm{H}$ & 36 & P-08 & (A) & $01 / 02 / 03$ & WAP & l & P03041:05 & Tank condition was normal. \\
\hline $\mathrm{H}$ & 36 & P-09 & (A) & $01 / 02 / 03$ & WAP & l & P03041:07 & Tank condition was normal. \\
\hline $\mathrm{H}$ & 36 & $P-10$ & (A) & $01 / 02 / 03$ & WAP & l & P03041:08 & Tank condition was normal. \\
\hline $\mathrm{H}$ & 36 & $\mathrm{P}-11$ & (A) & $01 / 02 / 03$ & WAP & l & P03041:09 & Tank condition was normal. \\
\hline $\mathrm{H}$ & 36 & $\mathrm{P}-12$ & (A) & $01 / 02 / 03$ & WAP & l & P03041:10 & Tank condition was normal. \\
\hline $\mathrm{H}$ & 36 & $P-13$ & (A) & $01 / 02 / 03$ & WAP & l & P03041:12 & Tank condition was normal. \\
\hline $\mathrm{H}$ & 36 & $P-14$ & (A) & $01 / 02 / 03$ & WAP & l & P03041:13 & Tank condition was normal. \\
\hline $\mathrm{H}$ & 37 & COP-106 & & $04 / 15 / 03$ & CCTV & l & 936 & $\begin{array}{l}\text { Inspection revealed salt deposits } \\
\text { on the GDL walls toward the } \\
\text { evaporator and tank. } \\
\text { Approximately } 25 \text { feet of the GDL } \\
\text { towards the evaporator was } \\
\text { reinspected after flushing. The } \\
\text { line was free of deposits. }\end{array}$ \\
\hline $\mathrm{H}$ & 37 & $A-01$ & (A) & $01 / 05 / 03$ & WAP & I & P03037:12 & Tank condition was normal. \\
\hline $\mathrm{H}$ & 37 & A-02 & (A) & $01 / 05 / 03$ & WAP & I & P03037:02 & Tank condition was normal. \\
\hline $\mathrm{H}$ & 37 & A-02 & (A) & 01/07/03 & CCTV & l & 907 & $\begin{array}{l}\text { The conductivity probe was } \\
\text { properly deployed per setpoint } \\
\text { document J-JX-G-0001. }\end{array}$ \\
\hline $\mathrm{H}$ & 37 & A-03 & (A) & $01 / 05 / 03$ & WAP & I & P03037:06 & Tank condition was normal. \\
\hline $\mathrm{H}$ & 37 & A-03 & (A) & 01/07/03 & CCTV & l & 907 & $\begin{array}{l}\text { The conductivity probe was } \\
\text { properly deployed per setpoint } \\
\text { document J-JX-G-0001. }\end{array}$ \\
\hline $\mathrm{H}$ & 37 & $\mathrm{~A}-04$ & (A) & $01 / 05 / 03$ & WAP & I & P03037:13 & Tank condition was normal. \\
\hline
\end{tabular}




\begin{tabular}{|c|c|c|c|c|c|c|c|c|}
\hline \multirow{2}{*}{$\frac{\text { AREA }}{\mathrm{H}}$} & \multirow{2}{*}{$\begin{array}{c}\begin{array}{c}\text { TANK OR } \\
\text { ANCILLARY }\end{array} \\
37\end{array}$} & \multicolumn{2}{|c|}{$\frac{\text { ACCESS OPENING }}{(A \text { OR I) }}$} & \multirow{2}{*}{$\frac{\text { DATE }}{01 / 07 / 03}$} & \multicolumn{3}{|c|}{$\frac{\text { INSPECTION METHOD }}{\text { IDENTIFICATION NUMBER }}$} & \multirow{2}{*}{$\begin{array}{l}\qquad \text { REMARKS } \\
\text { The conductivity probe was } \\
\text { properly deployed per setpoint } \\
\text { document J-JX-G-0001. }\end{array}$} \\
\hline & & A-04 & (A) & & CCTV & I & 907 & \\
\hline $\mathrm{H}$ & 37 & P-01 & (A) & 01/07/03 & DP & l & P03010:01-25 & Tank condition was normal. \\
\hline $\mathrm{H}$ & 37 & P-02 & (A) & 01/07/03 & DP & I & P03011:01-25 & Tank condition was normal. \\
\hline $\mathrm{H}$ & 37 & P-03 & (A) & $02 / 04 / 03$ & DP & I & P03072:01-27 & Tank condition was normal. \\
\hline $\mathrm{H}$ & 37 & P-04 & (A) & 01/07/03 & DP & I & P03012:01-25 & Tank condition was normal. \\
\hline $\mathrm{H}$ & 37 & P-05 & (A) & $01 / 05 / 03$ & WAP & I & P03037:01 & Tank condition was normal. \\
\hline $\mathrm{H}$ & 37 & P-06 & (A) & $01 / 05 / 03$ & WAP & I & P03037:03 & Tank condition was normal. \\
\hline $\mathrm{H}$ & 37 & P-07 & (A) & $01 / 05 / 03$ & WAP & I & P03037:04 & Tank condition was normal. \\
\hline $\mathrm{H}$ & 37 & P-08 & (A) & $01 / 05 / 03$ & WAP & I & P03037:05 & Tank condition was normal. \\
\hline $\mathrm{H}$ & 37 & P-09 & (A) & $01 / 05 / 03$ & WAP & I & P03037:07 & Tank condition was normal. \\
\hline $\mathrm{H}$ & 37 & $P-10$ & (A) & $01 / 05 / 03$ & WAP & I & P03037:08 & Tank condition was normal. \\
\hline $\mathrm{H}$ & 37 & $P-11$ & (A) & 01/05/03 & WAP & I & P03037:09 & Tank condition was normal. \\
\hline $\mathrm{H}$ & 37 & $\mathrm{P}-12$ & (A) & 01/05/03 & WAP & I & P03037:10 & Tank condition was normal. \\
\hline $\mathrm{H}$ & 37 & $P-13$ & (A) & $01 / 05 / 03$ & WAP & I & P03037:14 & Tank condition was normal. \\
\hline $\mathrm{H}$ & 37 & $\mathrm{P}-14$ & (A) & 01/05/03 & WAP & l & P03037:11 & Tank condition was normal. \\
\hline $\mathrm{H}$ & 37 & C-02 & (I) & 06/13/03 & CCTV & l & 953 & $\begin{array}{l}\text { Inspection of the BFV via the } \\
\text { catheter line revealed no unusual } \\
\text { conditions. The catheter line was } \\
\text { clean, and the valve was closed. }\end{array}$ \\
\hline $\mathrm{H}$ & 37 & C-02 & (I) & $06 / 18 / 03$ & CCTV & l & 953 & $\begin{array}{l}\text { Inspection documented the } \\
\text { conditions of the BFV seat. No } \\
\text { foreign material was observed. }\end{array}$ \\
\hline $\mathrm{H}$ & 37 & C-02 & (I) & 06/19/03 & CCTV & l & 953 & $\begin{array}{l}\text { CCTV was used to verify proper } \\
\text { BFV operation. Valve was } \\
\text { misaligned when placed in the } \\
\text { bottoms position. }\end{array}$ \\
\hline $\mathrm{H}$ & 37 & C-02 & (I) & $06 / 20 / 03$ & CCTV & l & 953 & $\begin{array}{l}\text { CCTV verified proper BFV } \\
\text { operation after valve stem was } \\
\text { reset. }\end{array}$ \\
\hline
\end{tabular}




\begin{tabular}{|c|c|c|c|c|}
\hline AREA & $\begin{array}{l}\text { TANK OR } \\
\text { ANCILLARY }\end{array}$ & $\frac{\text { ACCESS OPENING }}{(A \text { OR I })}$ & DATE & $\frac{\text { INSPECTION METHOD }}{\text { IDENTIFICATION NUMBER }}$ \\
\hline $\mathrm{H}$ & 37 & C-02 & 08/10/03 & CCTV \\
\hline
\end{tabular}

\section{REMARKS}

CCTV was used to monitor the BFV as it was manipulated. The valve did not move.

$\mathrm{H}$

37

C-02 (I)

08/12/03

CCTV /

953

$\mathrm{H}$

37

C-02 (I)

$08 / 17 / 03$

CCTV /

$\mathrm{H}$

37

C-02 (I)

08/17/03

CCTV I

953

$\mathrm{H}$

37

G (I)

03/06/03

CCTV /

892

CCTV I

1003

G (I)

$08 / 21 / 03$

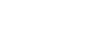

(1)

(1)

37
H (I) 04/25/03

$04 / 25 / 03$

$\mathrm{H}$
H 37

37
$\mathrm{H}$ (I) 06/16/03 CCTV /
Inspection verified that the shear pin was broken on the bottom universal joint of the BFV.

Inspection was made to document proper BFV operation after the shear pin was replaced. The valve was operating properly.

Inspection was made to verify proper BFV operation and perform a leak check. No unusual conditions or leaks were observed.

Inspection documented salt formations in the tank.

Approximately 6 inches of salt was observed on the cooling coils.

Salt formations were observed on the tank walls and center column. A small formation of salt suspended above the waste was observed spanning several coils.

Inspection documented salt formations in the tank. Cooling coils and the waste surface near the jet were free of salt accumulation due to a steam leak from the jet. All other cooling coils had approximately 2 - 3 inches of salt accumulation. The drive leg of the TTJ had some small accumulations, and the tank walls had less than one inch of accumulation. The waste surface, except beneath the jet, was covered with a crystallized salt layer.

Inspection of the tank interior revealed an irregular salt surface with pools of liquid located throughout the tank.

Inspection revealed salt formations 1 - 2 inches thick on the cooling coils, center column and tank walls. The surface of the waste was salt with some liquid visible in several locations. 


\section{TANK OR \\ AREA}

$\mathrm{H}$

37

$\frac{\text { ACCESS OPENING }}{\text { (A OR I) }}$

DATE

H (I)

07/20/03

CCTV

953

INSPECTION METHOD IDENTIFICATION NUMBER
CCTV I

1003

H (I) 08/21/03

$11 / 02 / 03$

$\mathrm{H} \quad(\mathrm{I})$

37

37
H (I)

$12 / 06 / 03$
CCTV

1019

CCTV / 1019

$\mathrm{H}$

H (I)

$12 / 07 / 03$
CCTV

\section{REMARKS}

Inspection documented the tank conditions and the reel tape. Liquid was observed throughout the tank. Salt formations were observed on the tank walls, center column and on top of several cooling coil loops. The reel tape was operating properly. No unusual conditions were observed.

Inspection was made to investigate level discrepancies. An irregular salt surface with some liquid was observed.

Approximately 3 inches of salt formations were observed on the exposed coils.

Inspection documented conditions beneath the riser. Salt formations were observed on the cooling coils, and the waste surface was liquid. No unusual conditions were observed.

Inspection was made to document conditions in the tank. Salt formations of approximately 8 inches were observed on the cooling coils and 2 - 3 inches of salt was observed on the tank wall. The surface of the waste was liquid. No salt mounds or unusual conditions were observed.

Inspection was made due to waste level discrepancies during transfer. Approximately 8 inches of salt accumulation were observed on the cooling coils and 2 - 3 inches of salt was observed on the tank wall. The waste was salt with some areas of liquid observed and appeared to be uniform across the tank. No salt mounds or unusual conditions were observed. 


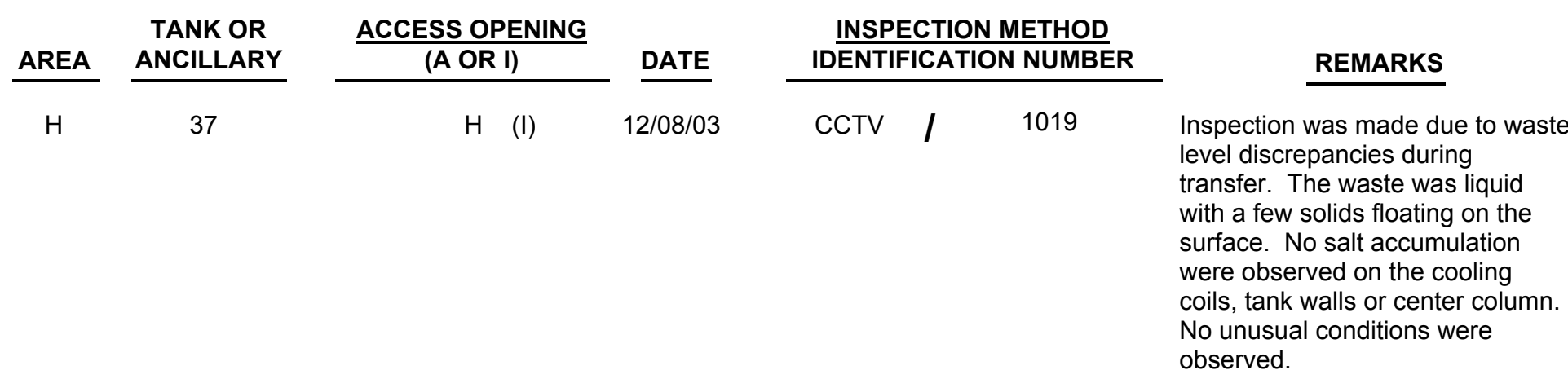

Inspection document conditions in the tank. Saltcake was observed throughout the tank with small formations of salt observed on some cooling coils near the TTJ and downcomer. Liquid was observed near the TTJ. No unusual conditions were observed.

Inspection document conditions in the tank. Liquid covered approximately $95 \%$ of the waste surface. No unusual conditions were observed.

Inspection documented condition of the GDL prior to cleaning with a high pressure water jet.

Approximately 25 feet of the line towards Tank 38 was inspected. A uniform coating covered the entire interior surface, with several areas having $30 \%-60 \%$ blockage.

Inspection of the GDL after initial cleaning revealed that most of the deposits remained.

CCTV documented the condition of approximately 23 feet of the GDL towards Tank 38 after the second cleaning. A thin coating remained on the pipe wall.

CCTV documented the condition of approximately 23 feet of the GDL towards Tank 38 and approximately 20 feet towards the $2 \mathrm{H}$ Evaporator after the third cleaning. A thin coating remained on the pipe wall. 


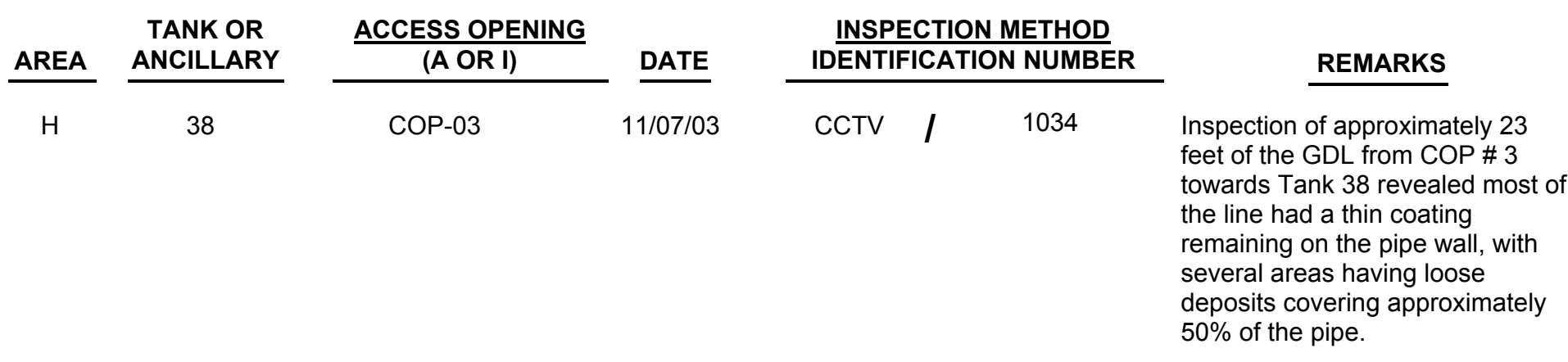

$\mathrm{H}$

38

8
COP-03

COP -03

LDB-01

A-01 (A)

A-02

A-02

(A)

01/11/03

A-03

(A)

01/09/03

A-03 (A)

$01 / 11 / 03$

A-04 (A) 01/09/03

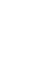

A-04 (A)

01/11/03

CCTV

907
1034

CCTV

1034

CCTV

964

WAP / P03021:01

WAP / P03021:02

CCTV / $\quad 907$

WAP

P03021:03

CCTV /

907

WAP

P03021:04
CCTV

Inspection of approximately 20 feet of the GDL from COP \# 3 towards $2 \mathrm{H}$ Evaporator revealed sections of the pipe completely clean, with other sections having a thin coating remaining on the pipe wall. No blockages were observed.

Inspection of approximately 25 feet of the GDL from COP \# 3 towards Tank 38 revealed a thin coating remained on the pipe wall.

The conductivity probe was properly deployed per setpoint document J-JX-G-0001.

Tank condition was normal.

Tank condition was normal.

The conductivity probe was properly deployed per setpoint document J-JX-G-0001.

Tank condition was normal.

The conductivity probe was properly deployed per setpoint document J-JX-G-0001.

Tank condition was normal. Stains and marks on the ventilation duct and annulus floor had increased due to the inleakage of water.

The conductivity probe was properly deployed per setpoint document J-JX-G-0001.

DP / P03028:01-27 Tank condition was normal.

DP / P03029:01-27 Tank condition was normal. 


\begin{tabular}{|c|c|c|c|c|c|c|c|c|}
\hline \multirow{2}{*}{$\frac{\text { AREA }}{H}$} & \multirow{2}{*}{$\begin{array}{c}\begin{array}{c}\text { TANK OR } \\
\text { ANCILLARY }\end{array} \\
38\end{array}$} & \multicolumn{2}{|c|}{$\frac{\text { ACCESS OPENING }}{(A \text { OR I) }}$} & \multirow{2}{*}{$\frac{\text { DATE }}{01 / 11 / 03}$} & \multicolumn{3}{|c|}{$\frac{\text { INSPECTION METHOD }}{\text { IDENTIFICATION NUMBER }}$} & \multirow{2}{*}{$\frac{\text { REMARKS }}{\text { Tank condition was normal. }}$} \\
\hline & & P-03 & (A) & & DP & l & P03030:01-27 & \\
\hline $\mathrm{H}$ & 38 & P-04 & (A) & $01 / 11 / 03$ & DP & l & P03031:01-27 & Tank condition was normal. \\
\hline $\mathrm{H}$ & 38 & P-05 & (A) & $01 / 09 / 03$ & WAP & I & P03021:05 & Tank condition was normal. \\
\hline $\mathrm{H}$ & 38 & P-06 & (A) & $01 / 09 / 03$ & WAP & I & P03021:06 & Tank condition was normal. \\
\hline $\mathrm{H}$ & 38 & P-07 & (A) & 01/09/03 & WAP & I & P03021:07 & Tank condition was normal. \\
\hline $\mathrm{H}$ & 38 & P-08 & (A) & $01 / 09 / 03$ & WAP & I & P03021:08 & Tank condition was normal. \\
\hline $\mathrm{H}$ & 38 & P-09 & (A) & $01 / 09 / 03$ & WAP & l & P03021:09 & Tank condition was normal. \\
\hline $\mathrm{H}$ & 38 & $P-10$ & (A) & $01 / 09 / 03$ & WAP & I & P03021:10 & Tank condition was normal. \\
\hline $\mathrm{H}$ & 38 & $P-11$ & (A) & $01 / 09 / 03$ & WAP & I & P03021:11 & Tank condition was normal. \\
\hline $\mathrm{H}$ & 38 & P-12 & (A) & 01/09/03 & WAP & I & P03021:12 & Tank condition was normal. \\
\hline $\mathrm{H}$ & 38 & $P-13$ & (A) & 01/09/03 & WAP & I & P03021:13 & Tank condition was normal. \\
\hline $\mathrm{H}$ & 38 & $\mathrm{P}-14$ & (A) & 01/09/03 & WAP & l & P03021:14 & Tank condition was normal. \\
\hline $\mathrm{H}$ & 39 & LDB-01 & & $04 / 29 / 03$ & CCTV & I & $822 \mathrm{~A}$ & $\begin{array}{l}\text { The conductivity probe was } \\
\text { properly deployed per setpoint } \\
\text { document J-JX-G-0001. }\end{array}$ \\
\hline $\mathrm{H}$ & 39 & LDB-01 & & $10 / 13 / 03$ & CCTV & I & 964 & $\begin{array}{l}\text { The conductivity probe was } \\
\text { properly deployed per setpoint } \\
\text { document J-JX-G-0001. }\end{array}$ \\
\hline $\mathrm{H}$ & 39 & $\begin{array}{l}\text { Underliner } \\
\text { Sump }\end{array}$ & & 09/06/03 & CCTV & I & 998 & $\begin{array}{l}\text { Inspection revealed that the } \\
\text { conductivity probe is resting in } \\
\text { standing liquid and mud. Rust } \\
\text { and debris were also observed in } \\
\text { the sump. }\end{array}$ \\
\hline $\mathrm{H}$ & 39 & $\begin{array}{r}\text { Underliner } \\
\text { Sump }\end{array}$ & & 09/07/03 & CCTV & I & 998 & $\begin{array}{l}\text { Inspection revealed no liquid or } \\
\text { debris after cleaning. }\end{array}$ \\
\hline $\mathrm{H}$ & 39 & $\begin{array}{r}\text { Underliner } \\
\text { crumn }\end{array}$ & & $09 / 15 / 03$ & CCTV & I & $822 \mathrm{~A}$ & $\begin{array}{l}\text { Inspection was made to document } \\
\text { conditions in the underliner sump. } \\
\text { Approximately } 3 \text { inches of liquid } \\
\text { was observed in the sump, and } \\
\text { the conductivity probe was } \\
\text { contacting the liquid. }\end{array}$ \\
\hline
\end{tabular}




\begin{tabular}{|c|c|c|c|c|c|c|c|c|}
\hline \multirow{2}{*}{$\frac{\text { AREA }}{H}$} & \multirow{2}{*}{$\begin{array}{c}\begin{array}{c}\text { TANK OR } \\
\text { ANCILLARY }\end{array} \\
39\end{array}$} & \multicolumn{2}{|c|}{$\frac{\text { ACCESS OPENING }}{(A \text { OR I) }}$} & \multirow{2}{*}{$\frac{\text { DATE }}{10 / 21 / 03}$} & \multicolumn{3}{|c|}{$\frac{\text { INSPECTION METHOD }}{\text { IDENTIFICATION NUMBER }}$} & \multirow{2}{*}{$\begin{array}{l}\text { REMARKS } \\
\text { Inspection was made to document } \\
\text { conditions and set the conductivity } \\
\text { probe. Mud and liquid were } \\
\text { observed in the sump. The } \\
\text { conductivity probe was set on the } \\
\text { bottom of the sump causing the } \\
\text { conductivity probe to alarm. }\end{array}$} \\
\hline & & $\begin{array}{l}\text { Underliner } \\
\text { Sump }\end{array}$ & & & CCTV & l & 998 & \\
\hline $\mathrm{H}$ & 39 & $A-01$ & (A) & $01 / 11 / 03$ & WAP & l & P03044:11 & Tank condition was normal. \\
\hline $\mathrm{H}$ & 39 & A-02 & (A) & 01/09/03 & WAP & l & P03044:06 & Tank condition was normal. \\
\hline $\mathrm{H}$ & 39 & A-02 & (A) & $01 / 11 / 03$ & CCTV & l & 907 & $\begin{array}{l}\text { The conductivity probe was } \\
\text { properly deployed per setpoint } \\
\text { document J-JX-G-0001. }\end{array}$ \\
\hline $\mathrm{H}$ & 39 & $\mathrm{~A}-03$ & (A) & 01/09/03 & WAP & I & P03044:07 & Tank condition was normal. \\
\hline $\mathrm{H}$ & 39 & A-03 & (A) & $01 / 11 / 03$ & CCTV & l & 907 & $\begin{array}{l}\text { The conductivity probe was } \\
\text { properly deployed per setpoint } \\
\text { document J-JX-G-0001. }\end{array}$ \\
\hline $\mathrm{H}$ & 39 & $A-04$ & (A) & 01/09/03 & WAP & l & P03044:08 & Tank condition was normal. \\
\hline $\mathrm{H}$ & 39 & $A-04$ & (A) & $01 / 11 / 03$ & CCTV & l & 907 & $\begin{array}{l}\text { The conductivity probe was } \\
\text { properly deployed per setpoint } \\
\text { document J-JX-G-0001. }\end{array}$ \\
\hline $\mathrm{H}$ & 39 & $\mathrm{P}-01$ & (A) & 01/09/03 & WAP & l & P03044:01 & Tank condition was normal. \\
\hline $\mathrm{H}$ & 39 & P-02 & (A) & $01 / 11 / 03$ & DP & l & P03032:01-27 & Tank condition was normal. \\
\hline $\mathrm{H}$ & 39 & $\mathrm{P}-03$ & (A) & 01/09/03 & WAP & l & P03044:02 & Tank condition was normal. \\
\hline $\mathrm{H}$ & 39 & $\mathrm{P}-04$ & (A) & $01 / 11 / 03$ & DP & l & P03033:01-27 & Tank condition was normal. \\
\hline $\mathrm{H}$ & 39 & P-05 & (A) & 01/09/03 & WAP & l & P03044:03 & Tank condition was normal. \\
\hline $\mathrm{H}$ & 39 & P-06 & (A) & $01 / 11 / 03$ & DP & l & P03034:01-27 & Tank condition was normal. \\
\hline $\mathrm{H}$ & 39 & P-07 & (A) & 01/09/03 & WAP & l & P03044:04 & Tank condition was normal. \\
\hline $\mathrm{H}$ & 39 & P-08 & (A) & 01/09/03 & WAP & l & P03044:05 & Tank condition was normal. \\
\hline $\mathrm{H}$ & 39 & P-09 & (A) & $01 / 11 / 03$ & DP & l & P03035:01-27 & Tank condition was normal. \\
\hline $\mathrm{H}$ & 39 & P-10 & (A) & 02/06/03 & DP & l & P03073:01-47 & $\begin{array}{l}\text { Tank condition was normal. A } \\
\text { baseline inspection of the } \\
\text { secondary vessel wall was normal. }\end{array}$ \\
\hline $\mathrm{H}$ & 39 & $\mathrm{P}-11$ & (A) & $01 / 09 / 03$ & WAP & l & P03044:12 & Tank condition was normal. \\
\hline
\end{tabular}




\begin{tabular}{|c|c|c|c|c|c|c|c|}
\hline \multirow{2}{*}{$\frac{\text { AREA }}{\mathrm{H}}$} & \multirow{2}{*}{$\begin{array}{c}\begin{array}{c}\text { TANK OR } \\
\text { ANCILLARY }\end{array} \\
39\end{array}$} & $\frac{\text { ACCESS OPENING }}{(A \text { OR I) }}$ & \multirow{2}{*}{$\frac{\text { DATE }}{01 / 11 / 03}$} & \multicolumn{3}{|c|}{$\frac{\text { INSPECTION METHOD }}{\text { IDENTIFICATION NUMBER }}$} & \multirow{2}{*}{$\frac{\text { REMARKS }}{\text { Tank condition was normal. }}$} \\
\hline & & $\mathrm{P}-12 \quad(\mathrm{~A})$ & & WAP & l & P03044:09 & \\
\hline $\mathrm{H}$ & 39 & $\mathrm{P}-13 \quad(\mathrm{~A})$ & $01 / 11 / 03$ & WAP & l & P03044:10 & Tank condition was normal. \\
\hline $\mathrm{H}$ & 39 & $\mathrm{P}-14 \quad(\mathrm{~A})$ & $01 / 09 / 03$ & WAP & I & P03044:13 & Tank condition was normal. \\
\hline $\mathrm{H}$ & 39 & $\mathrm{H} \quad(\mathrm{I})$ & $02 / 20 / 03$ & CCTV & l & $822 \mathrm{~A}$ & $\begin{array}{l}\text { The HLLCP was not visible when } \\
\text { deployed. The probe was raised, } \\
\text { viewed and lowered to the } \\
\text { setpoint. There appeared to be no } \\
\text { obstructions which prevented the } \\
\text { probe from being deployed } \\
\text { properly. }\end{array}$ \\
\hline
\end{tabular}

$\mathrm{H}$

39

$\mathrm{H}$

$\mathrm{H}$

$\mathrm{H}$

$\mathrm{H}$

$\mathrm{H}$

40

40

40

40

$\mathrm{H}$
H (I)

$06 / 18 / 03$

CCTV

$822 \mathrm{~A}$

H (I)

08/05/03

$04 / 24 / 03$

LDB-01

Underliner

Sump

A-01

(A)

01/09/03

A-02 (A)

01/09/03

WAP / P03038:02

CCTV

I

998

CCTV I

908

$09 / 15 / 03$

CCTV

I

946

WAP / P03038:01

$\mathrm{H}$

A-02 (A)

04/04/03
$01 / 12 / 03$

CCTV

907

$\mathrm{A}-02 \quad(\mathrm{~A})$
(1)
Inspection verified that the HLLCP cable was unobstructed. The HLLCP was not visible; it was hanging behind a cooling coil. The HLLCP was properly positioned.

CCTV was used to leak check transfer jet. No leaks were observed.

CCTV was used to document conditions in the LDB after a conductivity probe alarm was received. Mud and liquid was observed in the LDB causing the conductivity probe to alarm.

Inspection documented conditions in the underliner sump.

Approximately 3 inches of liquid was observed in the sump and mud on the bottom.

Tank condition was normal.

Tank condition was normal. Stains on the secondary vessel wall appeared to be due to the inleakage of water, additional inspection will be performed to investigate.

The conductivity probe was properly deployed per setpoint document J-JX-G-0001.

Secondary vessel wall was normal. Stains observed on scheduled inspection are no longer visible and were most likely from the inleakage of rainwater that has evaporated. 


\begin{tabular}{|c|c|c|c|c|c|c|c|c|}
\hline \multirow{2}{*}{$\frac{\text { AREA }}{\mathrm{H}}$} & \multirow{2}{*}{$\begin{array}{c}\begin{array}{c}\text { TANK OR } \\
\text { ANCILLARY }\end{array} \\
40\end{array}$} & \multicolumn{2}{|c|}{$\frac{\text { ACCESS OPENING }}{(A \text { OR I) }}$} & \multirow{2}{*}{$\frac{\text { DATE }}{01 / 09 / 03}$} & \multicolumn{3}{|c|}{$\frac{\text { INSPECTION METHOD }}{\text { IDENTIFICATION NUMBER }}$} & \multirow{2}{*}{$\frac{\text { REMARKS }}{\text { Tank condition was normal. }}$} \\
\hline & & $\mathrm{A}-03$ & (A) & & WAP & l & P03038:03 & \\
\hline $\mathrm{H}$ & 40 & $\mathrm{~A}-03$ & (A) & $01 / 12 / 03$ & CCTV & l & 907 & $\begin{array}{l}\text { The conductivity probe was } \\
\text { properly deployed per setpoint } \\
\text { document J-JX-G-0001. }\end{array}$ \\
\hline $\mathrm{H}$ & 40 & A-04 & (A) & 01/09/03 & WAP & I & P03038:04 & Tank condition was normal. \\
\hline $\mathrm{H}$ & 40 & A-04 & (A) & $01 / 12 / 03$ & CCTV & l & 907 & $\begin{array}{l}\text { The conductivity probe was } \\
\text { properly deployed per setpoint } \\
\text { document J-JX-G-0001. }\end{array}$ \\
\hline $\mathrm{H}$ & 40 & Center & (A) & 09/16/03 & CCTV & I & 946 & $\begin{array}{l}\text { CCTV was used to document } \\
\text { conditions and facilitate remote } \\
\text { operations to remove obstruction } \\
\text { beneath the center annulus } \\
\text { conductivity probe standpipe. The } \\
\text { obstruction was not removed and } \\
\text { the standpipe remained plugged. }\end{array}$ \\
\hline $\mathrm{H}$ & 40 & P-01 & (A) & 01/09/03 & WAP & I & P03038:05 & Tank condition was normal. \\
\hline $\mathrm{H}$ & 40 & P-02 & (A) & $01 / 12 / 03$ & DP & I & P03092:01-25 & Tank condition was normal. \\
\hline $\mathrm{H}$ & 40 & $\mathrm{P}-03$ & (A) & 01/09/03 & WAP & I & P03038:06 & Tank condition was normal. \\
\hline $\mathrm{H}$ & 40 & P-04 & (A) & 01/12/03 & DP & I & P03090:01-27 & Tank condition was normal. \\
\hline $\mathrm{H}$ & 40 & P-05 & (A) & 01/09/03 & WAP & I & P03038:07 & Tank condition was normal. \\
\hline $\mathrm{H}$ & 40 & P-06 & (A) & $01 / 12 / 03$ & $\mathrm{DP}$ & I & P03094:01-27 & Tank condition was normal. \\
\hline $\mathrm{H}$ & 40 & P-07 & (A) & 01/09/03 & WAP & I & P03038:08 & Tank condition was normal. \\
\hline $\mathrm{H}$ & 40 & $\mathrm{P}-08$ & (A) & $01 / 12 / 03$ & WAP & I & P03038:14 & Tank condition was normal. \\
\hline $\mathrm{H}$ & 40 & P-09 & (A) & 01/09/03 & WAP & I & P03038:09 & Tank condition was normal. \\
\hline $\mathrm{H}$ & 40 & $P-10$ & (A) & $01 / 12 / 03$ & DP & I & P03093:01-25 & Tank condition was normal. \\
\hline $\mathrm{H}$ & 40 & $P-11$ & (A) & 01/09/03 & WAP & I & P03038:10 & Tank condition was normal. \\
\hline $\mathrm{H}$ & 40 & $\mathrm{P}-12$ & (A) & $01 / 12 / 03$ & DP & I & P03091:01-25 & Tank condition was normal. \\
\hline $\mathrm{H}$ & 40 & $P-13$ & (A) & $01 / 09 / 03$ & WAP & I & P03038:11 & Tank condition was normal. \\
\hline $\mathrm{H}$ & 40 & $P-14$ & (A) & 01/09/03 & WAP & I & P03038:12 & Tank condition was normal. \\
\hline $\mathrm{H}$ & 40 & B-03 & (I) & 02/20/03 & CCTV & I & 813 & $\begin{array}{l}\text { Inspection verified that the HLLCP } \\
\text { was properly deployed. }\end{array}$ \\
\hline
\end{tabular}




\begin{tabular}{|c|c|c|c|c|}
\hline AREA & $\begin{array}{l}\text { TANK OR } \\
\text { ANCILLARY }\end{array}$ & $\frac{\text { ACCESS OPENING }}{(A \text { OR I) }}$ & DATE & $\frac{\text { INSPECTION METHOD }}{\text { IDENTIFICATION NUMBER }}$ \\
\hline $\mathrm{H}$ & 40 & C-03 (I) & $11 / 23 / 03$ & CCTV \\
\hline
\end{tabular}

\section{REMARKS}

CCTV was used to monitor the B-02 slurry pump for a bearing water leak. No obvious leakage was observed; however, air bubbles and liquid movement beside the pump at the liquid surface indicated that a leak may exist.

04/05/03 CCTV / 908

$05 / 21 / 03$

CCTV

964

06/05/03

CCTV

926

LDB-02

Underliner Sump

$10 / 20 / 03$

CCTV

989

Inspection performed after draining revealed no liquid in the LDB.

The conductivity probe was properly deployed per setpoint document J-JX-G-0001. The LDB.

The conductivity probe was properly deployed per setpoint document J-JX-G-0001 after cleaning and flushing the LDB. conditions in the sump and set
907

A-02 (A)

01/12/03

CCTV

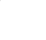

01/12/03

$\mathrm{A}-02 \quad(\mathrm{~A})$

A-03 (A)

01/12/03

$\mathrm{H}$

$\mathrm{H}$

$\mathrm{H}$
41

41

41

\begin{abstract}
A-03
\end{abstract}
(A)

A-04 (A)

(A)

01/12/03

A-0

(A)

$01 / 12 / 03$

P-01

(A)

01/12/03

P-02 (A)

02/04/03
WAP / P03040:01

CCTV / 907

WAP / P03040:05

CCTV / 907 probe was resting on debris in the

Inspection was made to document conductivity probe. Mud and liquid was observed in the sump. The probe was improperly deployed. The probe extended less than 1/4 inch below the end of the standpipe.

The conductivity probe was properly deployed per setpoint document J-JX-G-0001.

Tank condition was normal.

The conductivity probe was properly deployed per setpoint document J-JX-G-0001.

Tank condition was normal.

The conductivity probe was properly deployed per setpoint document J-JX-G-0001.

WAP / P03040:10 Tank condition was normal.

DP / P03042:01-28 Tank condition was normal.

DP / P03064:01-27

Tank condition was normal. 


\begin{tabular}{|c|c|c|c|c|c|c|c|c|}
\hline \multirow{2}{*}{$\frac{\text { AREA }}{\mathrm{H}}$} & \multirow{2}{*}{$\begin{array}{c}\begin{array}{c}\text { TANK OR } \\
\text { ANCILLARY }\end{array} \\
41\end{array}$} & \multicolumn{2}{|c|}{$\frac{\text { ACCESS OPENING }}{(\mathrm{A} \text { OR I) }}$} & \multirow{2}{*}{$\frac{\text { DATE }}{02 / 04 / 03}$} & \multicolumn{3}{|c|}{$\frac{\text { INSPECTION METHOD }}{\text { IDENTIFICATION NUMBER }}$} & \multirow{2}{*}{$\begin{array}{l}\text { REMARKS } \\
\text { Tank condition was normal. A } \\
\text { masselin cloth was observed on } \\
\text { the annulus floor beneath the } \\
\text { riser. }\end{array}$} \\
\hline & & P-03 & $(\mathrm{A})$ & & DP & I & P03063:01-27 & \\
\hline $\mathrm{H}$ & 41 & P-04 & $(\mathrm{A})$ & $01 / 12 / 03$ & DP & l & P03043:01-27 & Tank condition was normal. \\
\hline $\mathrm{H}$ & 41 & P-05 & (A) & $01 / 12 / 03$ & WAP & l & P03040:02 & Tank condition was normal. \\
\hline $\mathrm{H}$ & 41 & P-06 & $(\mathrm{A})$ & $01 / 12 / 03$ & WAP & I & P03040:03 & Tank condition was normal. \\
\hline $\mathrm{H}$ & 41 & P-07 & (A) & $01 / 12 / 03$ & WAP & l & P03040:04 & Tank condition was normal. \\
\hline $\mathrm{H}$ & 41 & P-08 & (A) & $01 / 12 / 03$ & WAP & l & P03040:06 & Tank condition was normal. \\
\hline $\mathrm{H}$ & 41 & P-09 & $(\mathrm{A})$ & $01 / 12 / 03$ & WAP & l & P03040:07 & Tank condition was normal. \\
\hline $\mathrm{H}$ & 41 & $P-10$ & (A) & $01 / 12 / 03$ & WAP & l & P03040:08 & Tank condition was normal. \\
\hline $\mathrm{H}$ & 41 & $P-11$ & (A) & $01 / 12 / 03$ & WAP & l & P03040:09 & Tank condition was normal. \\
\hline $\mathrm{H}$ & 41 & $\mathrm{P}-12$ & (A) & $01 / 12 / 03$ & WAP & l & P03040:11 & Tank condition was normal. \\
\hline $\mathrm{H}$ & 41 & $P-13$ & (A) & $01 / 12 / 03$ & WAP & l & P03040:12 & Tank condition was normal. \\
\hline $\mathrm{H}$ & 41 & $P-14$ & (A) & $01 / 12 / 03$ & WAP & l & P03040:13 & Tank condition was normal. \\
\hline $\mathrm{H}$ & 41 & B-02 & (I) & $06 / 23 / 03$ & CCTV & l & 987 & $\begin{array}{l}\text { Inspection documented the well } \\
\text { beneath the C-03 riser after water } \\
\text { additions. }\end{array}$ \\
\hline $\mathrm{H}$ & 41 & B-02 & (I) & $06 / 24 / 03$ & CCTV & l & 987 & $\begin{array}{l}\text { Inspection documented the void } \\
\text { created by salt dissolution around } \\
\text { the pump installed in the C-03 } \\
\text { riser. }\end{array}$ \\
\hline $\mathrm{H}$ & 41 & B-02 & (l) & 06/25/03 & CCTV & l & 988 & $\begin{array}{l}\text { Inspection documented the void } \\
\text { created by salt dissolution around } \\
\text { the pump installed in the C-03 } \\
\text { riser. }\end{array}$ \\
\hline $\mathrm{H}$ & 41 & B-02 & $(\mathrm{I})$ & 07/03/03 & CCTV & l & 988 & $\begin{array}{l}\text { Inspection documented tank } \\
\text { conditions. Salt was observed } \\
\text { throughout the tank except } \\
\text { beneath the C- } 03 \text { riser. Water } \\
\text { had filled the void around the } \\
\text { pump. A cooling coil (\#23) was } \\
\text { observed outside of the upper } \\
\text { guide. No other unusual } \\
\text { conditions were observed. }\end{array}$ \\
\hline
\end{tabular}




\begin{tabular}{|c|c|c|c|c|c|c|}
\hline \multirow{2}{*}{$\frac{\text { AREA }}{\mathrm{H}}$} & \multirow{2}{*}{$\begin{array}{c}\begin{array}{c}\text { TANK OR } \\
\text { ANCILLARY }\end{array} \\
41\end{array}$} & $\frac{\text { ACCESS OPENING }}{(A \text { OR I) }}$ & \multirow{2}{*}{$\frac{\text { DATE }}{07 / 04 / 03}$} & \multicolumn{2}{|c|}{$\frac{\text { INSPECTION METHOD }}{\text { IDENTIFICATION NUMBER }}$} & \multirow{2}{*}{$\begin{array}{l}\text { REMARKS } \\
\text { Inspection was performed to } \\
\text { document tank conditions during } \\
\text { water additions. Liquid was } \\
\text { observed throughout most of the } \\
\text { tank. No unusual conditions were } \\
\text { observed. }\end{array}$} \\
\hline & & B-02 (I) & & CCTV & 989 & \\
\hline
\end{tabular}

$\mathrm{H}$

$\mathrm{H}$

$\mathrm{H}$

$\mathrm{H}$

$\mathrm{H}$

$\mathrm{H}$

$\mathrm{H}$

$\mathrm{H}$

42

41

41

41

42

42
B-02 (I)

07/05/03

CCTV

989

B-02

(I)

07/06/03

CCTV

989

B-03 (I)

10/30/03

CCTV /

989

G (I)

$10 / 15 / 03$

CCTV I

989

$12 / 09 / 03$

Underliner

Sump

Underliner

Sump

Underliner

Sump

VB

09/16/03

CCTV I

854
Inspection was performed to document tank conditions. Liquid was observed throughout most of the tank. No unusual conditions were observed.

Inspection was performed to document tank conditions. Liquid was observed throughout most of the tank. No unusual conditions were observed.

Inspection documented the waste surface conditions in the tank. Liquid was observed throughout the tank with a salt layer forming. No salt was observed above the liquid level. No unusual conditions were observed.

Inspection documented the surface conditions of the waste. Liquid was observed throughout the tank with some salt crystals forming on the top. Salt was observed below the liquid surface. No unusual conditions were observed. The HLLCP was properly deployed.

Inspection documented conditions in the sump. Mud and water were observed.

Inspection documented conditions in the underliner sump after flushing. Liquid was observed in the sump.

Inspection verified that the conductivity probe was properly deployed.

Inspection was made to determine why the jumper in the valve box was stuck. The brace or clip on one end of the jumper is missing or misaligned. No other unusual conditions were observed.

Tank condition was normal. 


\begin{tabular}{|c|c|c|c|c|c|c|c|c|}
\hline \multirow{2}{*}{$\frac{\text { AREA }}{\mathrm{H}}$} & \multirow{2}{*}{$\begin{array}{c}\begin{array}{c}\text { TANK OR } \\
\text { ANCILLARY }\end{array} \\
42\end{array}$} & \multicolumn{2}{|c|}{$\frac{\text { ACCESS OPENING }}{(\text { A OR I) }}$} & \multirow{2}{*}{$\frac{\text { DATE }}{02 / 25 / 03}$} & \multicolumn{3}{|c|}{$\frac{\text { INSPECTION METHOD }}{\text { IDENTIFICATION NUMBER }}$} & \multirow{2}{*}{$\begin{array}{l}\qquad \text { REMARKS } \\
\qquad \begin{array}{l}\text { The conductivity probe was } \\
\text { properly deployed per setpoin } \\
\text { document J-JX-G-0001. }\end{array}\end{array}$} \\
\hline & & A-02 & (A) & & CCTV & l & 914 & \\
\hline $\mathrm{H}$ & 42 & A-02 & (A) & $02 / 27 / 03$ & WAP & l & P03101:08 & Tank condition was normal. \\
\hline $\mathrm{H}$ & 42 & A-03 & (A) & $02 / 25 / 03$ & CCTV & l & 914 & $\begin{array}{l}\text { The conductivity probe was } \\
\text { properly deployed per setpoin } \\
\text { document J-JX-G-0001. }\end{array}$ \\
\hline $\mathrm{H}$ & 42 & A-03 & (A) & $02 / 27 / 03$ & WAP & l & P03101:12 & Tank condition was normal. \\
\hline $\mathrm{H}$ & 42 & A-04 & (A) & $02 / 25 / 03$ & CCTV & l & 914 & $\begin{array}{l}\text { The conductivity probe was } \\
\text { properly deployed per setpoin } \\
\text { document J-JX-G-0001. }\end{array}$ \\
\hline $\mathrm{H}$ & 42 & A-04 & (A) & $02 / 27 / 03$ & WAP & l & P03101:01 & Tank condition was normal. \\
\hline $\mathrm{H}$ & 42 & P-01 & (A) & 07/30/03 & DP & l & P03155:01-27 & Tank condition was normal. \\
\hline $\mathrm{H}$ & 42 & P-02 & (A) & $07 / 30 / 03$ & DP & l & P03156:01-27 & Tank condition was normal. \\
\hline $\mathrm{H}$ & 42 & $\mathrm{P}-03$ & (A) & $02 / 27 / 03$ & WAP & l & P03101:07 & Tank condition was normal. \\
\hline $\mathrm{H}$ & 42 & P-04 & (A) & $02 / 27 / 03$ & WAP & l & P03101:09 & Tank condition was normal. \\
\hline $\mathrm{H}$ & 42 & P-05 & (A) & $02 / 27 / 03$ & WAP & l & P03101:14 & Tank condition was normal. \\
\hline $\mathrm{H}$ & 42 & P-06 & (A) & $02 / 27 / 03$ & WAP & l & P03101:10 & Tank condition was normal. \\
\hline $\mathrm{H}$ & 42 & P-07 & (A) & $02 / 27 / 03$ & WAP & l & P03101:11 & Tank condition was normal. \\
\hline $\mathrm{H}$ & 42 & $\mathrm{P}-08$ & (A) & $02 / 27 / 03$ & WAP & l & P03101:13 & Tank condition was normal. \\
\hline $\mathrm{H}$ & 42 & P-09 & (A) & $07 / 30 / 03$ & DP & l & P03157:01-28 & Tank condition was normal. \\
\hline $\mathrm{H}$ & 42 & $P-10$ & (A) & 07/30/03 & DP & l & P03158:01-27 & Tank condition was normal. \\
\hline $\mathrm{H}$ & 42 & $\mathrm{P}-11$ & (A) & $02 / 27 / 03$ & WAP & l & P03101:02 & Tank condition was normal. \\
\hline $\mathrm{H}$ & 42 & $\mathrm{P}-12$ & (A) & $02 / 27 / 03$ & WAP & l & P03101:03 & Tank condition was normal. \\
\hline $\mathrm{H}$ & 42 & $P-13$ & (A) & $02 / 27 / 03$ & WAP & l & P03101:04 & Tank condition was normal. \\
\hline $\mathrm{H}$ & 42 & P-14 & (A) & $02 / 27 / 03$ & WAP & I & P03101:05 & Tank condition was normal. \\
\hline
\end{tabular}




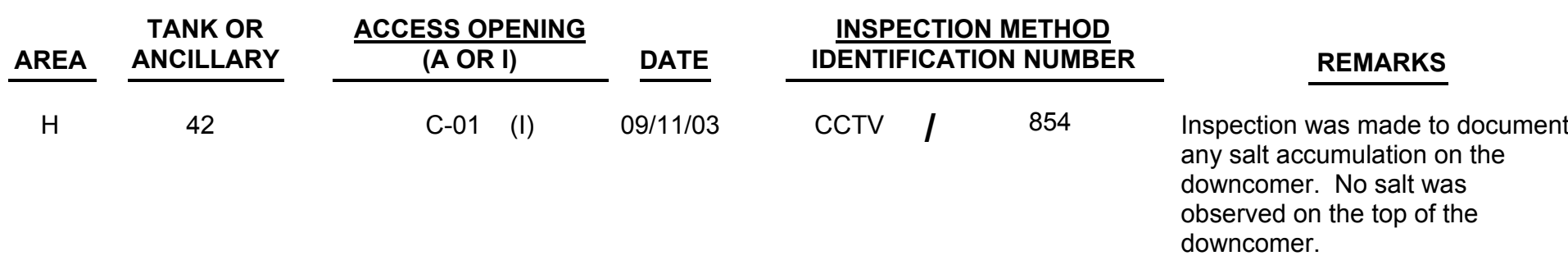

$\mathrm{H}$

Pipe

$\mathrm{H}$

$\mathrm{H}$

$\mathrm{H}$

$\mathrm{H}$

$\mathrm{H}$

$\mathrm{H}$

43

LDB-04

03/26/03

HELIUM /

HE-03-004

$$
\text { CCTV I }
$$

06/24/03

CCTV

821

03/19/03 CCTV / 908

03/24/03 CCTV / 908

$03 / 26 / 03$

CCTV

908
CCTV was used to inspect the vent line core pipe from the 242-16H Evaporator. No unusual conditions were observed.

CCTV was used to inspect the vent line core pipe from the 242-16H Evaporator. No unusual conditions were observed.

The conductivity probe was properly deployed per setpoint document J-JX-G-0001. Debris was observed in the bottom of the LDB.

09/29/03 CCTV / 964 properly deployed per setpoint document J-JX-G-0001.

Helium tracer testing was performed on the feed line from Tank 43 to the 242-16H evaporator. Elevated levels found in the tank annulus exhaust indicate a failure of the jacket where the transfer line passes through the annulus.

09/29/03 CCTV / 964

LDB-04

LDB-07

03/18/03
908
The conductivity probe was properly deployed per setpoint document J-JX-G-0001.

The conductivity probe was properly deployed per setpoint document J-JX-G-0001. 


\begin{tabular}{|c|c|c|c|c|c|c|c|}
\hline \multirow{2}{*}{$\frac{\text { AREA }}{\mathrm{H}}$} & \multirow{2}{*}{$\begin{array}{c}\begin{array}{c}\text { TANK OR } \\
\text { ANCILLARY }\end{array} \\
43\end{array}$} & \multirow{2}{*}{$\frac{\frac{\text { ACCESS OPENING }}{(\text { A OR I) }}}{\text { LDB- } 07}$} & \multirow{2}{*}{$\frac{\text { DATE }}{03 / 24 / 03}$} & \multicolumn{3}{|c|}{$\begin{array}{l}\text { INSPECTION METHOD } \\
\text { IDENTIFICATION NUMBER }\end{array}$} & \multirow{2}{*}{$\begin{array}{l}\qquad \text { REMARKS } \\
\qquad \begin{array}{l}\text { The conductivity probe was } \\
\text { properly deployed per setpoint } \\
\text { document J-JX-G-0001. }\end{array}\end{array}$} \\
\hline & & & & CCTV & I & 908 & \\
\hline $\mathrm{H}$ & 43 & LDB-07 & 09/23/03 & CCTV & I & 821 & $\begin{array}{l}\text { The conductivity probe was } \\
\text { properly deployed per setpoint } \\
\text { document J-JX-G-0001. }\end{array}$ \\
\hline $\mathrm{H}$ & 43 & $\begin{array}{l}\text { Underliner } \\
\text { Sump }\end{array}$ & 09/06/03 & CCTV & I & 821 & $\begin{array}{l}\text { The conductivity probe was not } \\
\text { visible due to the amount of liquid } \\
\text { in the sump. Rust, mud, liquid } \\
\text { and a piece of Tygon tubing were } \\
\text { observed in the sump. }\end{array}$ \\
\hline $\mathrm{H}$ & 43 & $\begin{array}{r}\text { Underliner } \\
\text { Sıımn }\end{array}$ & $10 / 22 / 03$ & CCTV & I & 1023 & $\begin{array}{l}\text { Inspection documented conditions } \\
\text { in the underliner sump after } \\
\text { flushing. Mud was observed in } \\
\text { the underliner sump and below the } \\
\text { conductivity probe standpipe. The } \\
\text { accumulation of mud beneath the } \\
\text { standpipe blocked access to the } \\
\text { sump. The conductivity probe } \\
\text { could not be deployed. }\end{array}$ \\
\hline $\mathrm{H}$ & 43 & $\begin{array}{l}\text { Underliner } \\
\text { Sump }\end{array}$ & $10 / 23 / 03$ & CCTV & I & 1023 & $\begin{array}{l}\text { Inspection documented conditions } \\
\text { in the underliner sump. Mud and } \\
\text { debris were observed beneath the } \\
\text { conductivity probe standpipe. }\end{array}$ \\
\hline
\end{tabular}

$\mathrm{H}$

Inspection documented conditions in the underliner sump. Mud, sand/silt, a piece of Tygon tubing and water was observed in the sump.

CCTV was used to facilitate removal of Tygon tubing and add flush water to clean underliner sump.

$$
\mathrm{H}
$$

Inspection documented conditions in the underliner sump. Mud, sand/silt and water were observed. The water level extended into the conductivity probe standpipe preventing conductivity probe deployment.

Tank condition was normal.

The conductivity probe was properly deployed per setpoint document J-JX-G-0001.

Tank condition was normal. 


\begin{tabular}{|c|c|c|c|c|c|c|c|c|}
\hline \multirow{2}{*}{$\frac{\text { AREA }}{H}$} & \multirow{2}{*}{$\begin{array}{c}\text { TANK OR } \\
\text { ANCILLARY } \\
43\end{array}$} & \multicolumn{2}{|c|}{$\frac{\text { ACCESS OPENING }}{(A \text { OR I) }}$} & \multirow{2}{*}{$\frac{\text { DATE }}{02 / 25 / 03}$} & \multicolumn{3}{|c|}{$\frac{\text { INSPECTION METHOD }}{\text { IDENTIFICATION NUMBER }}$} & \multirow{2}{*}{$\begin{array}{l}\qquad \text { REMARKS } \\
\qquad \begin{array}{l}\text { The conductivity probe was } \\
\text { properly deployed per setpoint } \\
\text { document J-JX-G-0001. }\end{array}\end{array}$} \\
\hline & & A-03 & (A) & & CCTV & I & 914 & \\
\hline $\mathrm{H}$ & 43 & A-03 & (A) & $02 / 27 / 03$ & WAP & l & P03102:05 & Tank condition was normal. \\
\hline $\mathrm{H}$ & 43 & A-04 & (A) & $02 / 25 / 03$ & CCTV & l & 914 & $\begin{array}{l}\text { The conductivity probe was } \\
\text { properly deployed per setpoint } \\
\text { document J-JX-G-0001. }\end{array}$ \\
\hline $\mathrm{H}$ & 43 & $A-04$ & (A) & $02 / 27 / 03$ & WAP & l & P03102:09 & Tank condition was normal. \\
\hline $\mathrm{H}$ & 43 & P-01 & (A) & 07/30/03 & DP & l & P03159:01-26 & Tank condition was normal. \\
\hline $\mathrm{H}$ & 43 & P-02 & (A) & $07 / 31 / 03$ & DP & l & P03160:01-27 & $\begin{array}{l}\text { Tank condition was normal. } \\
\text { Stains and marks on the primary } \\
\text { vessel wall had increased due to } \\
\text { the inleakage of water. }\end{array}$ \\
\hline $\mathrm{H}$ & 43 & P-03 & (A) & $07 / 31 / 03$ & DP & l & P03161:01-27 & $\begin{array}{l}\text { Tank condition was normal. } \\
\text { Stains and marks on the primary } \\
\text { vessel wall had increased due to } \\
\text { the inleakage of water. }\end{array}$ \\
\hline $\mathrm{H}$ & 43 & P-04 & (A) & 07/31/03 & DP & l & P03162:01-28 & $\begin{array}{l}\text { Tank condition was normal. } \\
\text { Stains and marks on the primary } \\
\text { vessel wall had increased due to } \\
\text { the inleakage of water. }\end{array}$ \\
\hline $\mathrm{H}$ & 43 & P-05 & (A) & $02 / 27 / 03$ & WAP & l & P03102:02 & Tank condition was normal. \\
\hline $\mathrm{H}$ & 43 & P-06 & (A) & $02 / 27 / 03$ & WAP & I & P03102:03 & Tank condition was normal. \\
\hline $\mathrm{H}$ & 43 & P-07 & (A) & $02 / 27 / 03$ & WAP & l & P03102:04 & Tank condition was normal. \\
\hline $\mathrm{H}$ & 43 & P-08 & (A) & $02 / 27 / 03$ & WAP & l & P03102:06 & Tank condition was normal. \\
\hline $\mathrm{H}$ & 43 & P-09 & (A) & $02 / 27 / 03$ & WAP & l & P03102:07 & Tank condition was normal. \\
\hline $\mathrm{H}$ & 43 & $P-10$ & (A) & $02 / 27 / 03$ & WAP & l & P03102:08 & Tank condition was normal. \\
\hline $\mathrm{H}$ & 43 & $\mathrm{P}-11$ & (A) & $02 / 27 / 03$ & WAP & l & P03102:10 & Tank condition was normal. \\
\hline $\mathrm{H}$ & 43 & $\mathrm{P}-12$ & (A) & $02 / 27 / 03$ & WAP & l & P03102:11 & Tank condition was normal. \\
\hline $\mathrm{H}$ & 43 & $P-13$ & (A) & $02 / 27 / 03$ & WAP & l & P03102:12 & Tank condition was normal. \\
\hline $\mathrm{H}$ & 43 & $\mathrm{P}-14$ & (A) & $02 / 27 / 03$ & WAP & l & P03102:13 & Tank condition was normal. \\
\hline $\mathrm{F}$ & 44 & & & $08 / 20 / 03$ & HELIUM & l & HE-03-009 & $\begin{array}{l}\text { A helium tracer test verified the } \\
\text { integrity of the the jacket } \\
\text { connector lines designated } \\
\text { JC4384 and JC4386. }\end{array}$ \\
\hline
\end{tabular}




\begin{tabular}{|c|c|c|c|c|c|c|}
\hline$\frac{\text { AREA }}{F}$ & \multirow{2}{*}{$\begin{array}{c}\begin{array}{c}\text { TANK OR } \\
\text { ANCILLARY }\end{array} \\
44\end{array}$} & \multirow[t]{2}{*}{$\frac{\text { ACCESS OPENING }}{(\text { A OR I) }}$} & \multirow{2}{*}{$\frac{\text { DATE }}{09 / 25 / 03}$} & \multicolumn{2}{|c|}{$\frac{\text { INSPECTION METHOD }}{\text { IDENTIFICATION NUMBER }}$} & \multirow{2}{*}{$\begin{array}{l}\text { REMARKS } \\
\text { Helium testing was performed on } \\
\text { the transfer line from FDB-04 to } \\
\text { Tanks } 44 \text { and } 45 \text {. A small amount } \\
\text { of helium was detected at FDB-4 } \\
\text { exhaust indicating leak through at } \\
\text { the overflow pipe of LDB-08. }\end{array}$} \\
\hline & & & & HELIUM / & HE-03-010 & \\
\hline
\end{tabular}

F

44

44

$\mathrm{F}$

$\mathrm{F}$

F

F

$\mathrm{F}$

F

$\mathrm{F}$

$\mathrm{F}$

F

$\mathrm{F}$

$\mathrm{F}$

$\mathrm{F}$
44

44

44

44

44

44

44

44

44

44

44
09/26/03 HELIUM / HE-03-011

09/26/03

LDB-01

LDB-01

LDB-02

LDB-03

\begin{abstract}
A-01 (A)
\end{abstract}
A-02

(A)

A-02 (A)

A-03 (A)

A-03 (A)

A-04 (A)

A-04 (A)

P-01

(A)
$02 / 25 / 03$

$02 / 25 / 03$

02/25/03

02/05/03

09/26/03

05/19/03

03/04/03

02/25/03

02/25/03

02/25/03

$02 / 25 / 03$

$02 / 25 / 03$
HELIUM / HE-03-012

CCTV I 908

CCTV I 964

CCTV I 964

CCTV I 908

CCTV I 914

WAP / P03098:02

$\begin{array}{ll}\text { CCTV I } & 914\end{array}$

WAP I P03098:03

$\begin{array}{lll}\text { CCTV I } & 914\end{array}$

WAP / P03098:04

WAP I P03098:05
WAP / P03098:01
Helium tracer testing verified the integrity of the transfer line from Tank 44 to FDB-04.

Helium tracer testing verified the integrity of the jacket connector lines designated JC4384 and JC4386.

The conductivity probe was properly deployed per setpoint document J-JX-G-0001.

The conductivity probe was properly deployed per setpoint document J-JX-G-0001.

The conductivity probe was properly deployed per setpoint document J-JX-G-0001.

The conductivity probe was properly deployed per setpoint document J-JX-G-0001.

Tank condition was normal.

The conductivity probe was properly deployed per setpoint document J-JX-G-0001.

Tank condition was normal.

The conductivity probe was properly deployed per setpoint document J-JX-G-0001.

Tank condition was normal.

The conductivity probe was properly deployed per setpoint document J-JX-G-0001.

Tank condition was normal.

Tank condition was normal. 


\begin{tabular}{|c|c|c|c|c|c|c|c|c|}
\hline \multirow{2}{*}{$\frac{\text { AREA }}{F}$} & \multirow{2}{*}{$\begin{array}{c}\text { TANK OR } \\
\text { ANCILLARY } \\
44\end{array}$} & \multicolumn{2}{|c|}{$\frac{\text { ACCESS OPENING }}{(A \text { OR I) }}$} & \multirow{2}{*}{$\frac{\text { DATE }}{02 / 25 / 03}$} & \multicolumn{3}{|c|}{$\frac{\text { INSPECTION METHOD }}{\text { IDENTIFICATION NUMBER }}$} & \multirow{2}{*}{$\frac{\text { REMARKS }}{\text { Tank condition was normal. }}$} \\
\hline & & $\mathrm{P}-02$ & (A) & & WAP & l & P03098:06 & \\
\hline $\mathrm{F}$ & 44 & $\mathrm{P}-03$ & (A) & $05 / 13 / 03$ & DP & l & P03109:01-27 & $\begin{array}{l}\text { Tank condition was normal. } \\
\text { Stains and marks on the } \\
\text { secondary vessel wall had } \\
\text { increased due to the the inleakage } \\
\text { of water. }\end{array}$ \\
\hline $\mathrm{F}$ & 44 & P-04 & (A) & 05/13/03 & DP & I & P03110:01-27 & Tank condition was normal. \\
\hline $\mathrm{F}$ & 44 & P-05 & (A) & $05 / 13 / 03$ & DP & I & P03111:01-28 & Tank condition was normal. \\
\hline $\mathrm{F}$ & 44 & P-06 & (A) & $05 / 13 / 03$ & DP & I & P03108:01-28 & Tank condition was normal. \\
\hline $\mathrm{F}$ & 44 & $\mathrm{P}-07$ & (A) & $05 / 13 / 03$ & DP & I & P03112:01-27 & Tank condition was normal. \\
\hline $\mathrm{F}$ & 44 & $\mathrm{P}-08$ & (A) & $02 / 25 / 03$ & WAP & I & P03098:07 & Tank condition was normal. \\
\hline $\mathrm{F}$ & 44 & P-09 & (A) & $02 / 25 / 03$ & WAP & l & P03098:08 & Tank condition was normal. \\
\hline $\mathrm{F}$ & 44 & P-10 & (A) & $02 / 25 / 03$ & WAP & I & P03098:09 & Tank condition was normal. \\
\hline $\mathrm{F}$ & 44 & $\mathrm{P}-11$ & (A) & $02 / 25 / 03$ & WAP & I & P03098:10 & Tank condition was normal. \\
\hline $\mathrm{F}$ & 44 & $\mathrm{P}-12$ & (A) & $02 / 25 / 03$ & WAP & I & P03098:11 & Tank condition was normal. \\
\hline $\mathrm{F}$ & 44 & $P-13$ & (A) & $02 / 25 / 03$ & WAP & I & P03098:12 & Tank condition was normal. \\
\hline $\mathrm{F}$ & 44 & P-14 & (A) & $02 / 25 / 03$ & WAP & I & P03098:13 & Tank condition was normal. \\
\hline $\mathrm{F}$ & 45 & & & 09/25/03 & HELIUM & I & HE-03-010 & $\begin{array}{l}\text { Helium testing was performed on } \\
\text { the transfer line from FDB- } 04 \text { to } \\
\text { Tanks } 44 \text { and } 45 \text {. A small amount } \\
\text { of helium was detected at FDB- } 4 \\
\text { exhaust indicating leak through at } \\
\text { the overflow pipe of LDB-08. }\end{array}$ \\
\hline $\mathrm{F}$ & 45 & LDB-01 & & $04 / 15 / 03$ & CCTV & l & 908 & $\begin{array}{l}\text { The conductivity probe was } \\
\text { properly deployed per setpoint } \\
\text { document J-JX-G-0001. }\end{array}$ \\
\hline $\mathrm{F}$ & 45 & LDB-02 & & $03 / 04 / 03$ & CCTV & l & 908 & $\begin{array}{l}\text { The conductivity probe was } \\
\text { deployed per setpoint document } \\
\text { J-JX-G-0001. }\end{array}$ \\
\hline $\mathrm{F}$ & 45 & $A-01$ & $(\mathrm{~A})$ & $02 / 25 / 03$ & WAP & l & P03095:01 & Tank condition was normal. \\
\hline $\mathrm{F}$ & 45 & A-02 & (A) & $02 / 25 / 03$ & CCTV & l & 914 & $\begin{array}{l}\text { The conductivity probe was } \\
\text { properly deployed per setpoint } \\
\text { document J-JX-G-0001. }\end{array}$ \\
\hline
\end{tabular}




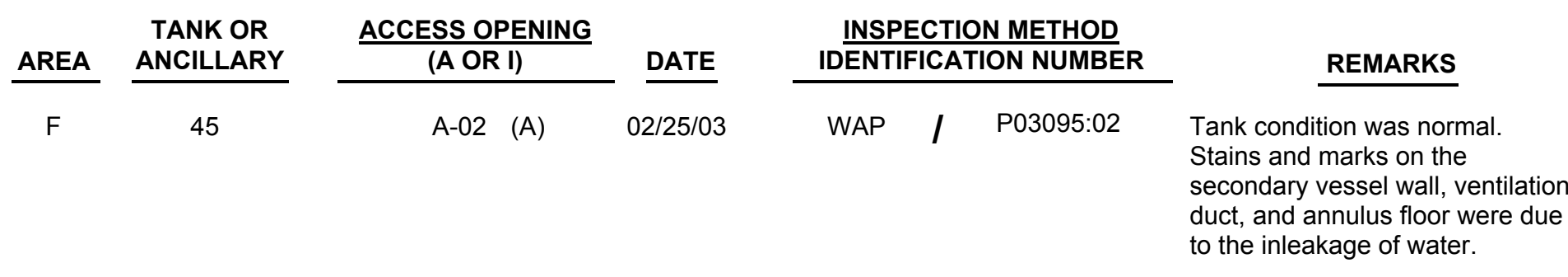

F

45

45

45

F

F

F

F

F

F

F

F

F

F

F

F

F

F

F

F

45

45

45

45

45

45

45

45

45

45

45

45

45

45

45
A-03 (A) $\quad 02 / 25 / 03$

A-03 (A)

A-04 (A)

$02 / 25 / 03$
A-04 (A) $\quad 02 / 25 / 03$

P-01 (A) 02/25/03

P-02 (A)

$\mathrm{P}-03 \quad(A)$

$\mathrm{P}-04 \quad(A)$

$\mathrm{P}-05 \quad(A)$

P-06 (A)

$\mathrm{P}-07 \quad(A)$

$\mathrm{P}-08 \quad(A)$

P-09 (A)

P-10 (A)

P-11 (A)

02/25/03

$\mathrm{P}-12$ (A)

02/25/03

$\mathrm{P}-13 \quad(\mathrm{~A})$

02/25/03

P-14 (A) 02/25/03
CCTV / 914

WAP / P03095:03

CCTV / 914

WAP I P03095.04

WAP / P03095:05

WAP / P03095:06 Tank condition was normal.

$\mathrm{DP} \quad / \quad \mathrm{P} 03103: 01-26$

$\mathrm{DP} \quad$ / P03104:01-28

$\mathrm{DP} \quad / \quad \mathrm{P} 03105: 01-27$

$\mathrm{DP} \quad$ / P03106:01-28

DP / P03107:01-27

WAP / P03095:07

WAP / P03095:08

WAP / P03095:09

WAP / P03095:10

WAP I P03095:11

Tank condition was normal.

Stains and marks on the secondary vessel wall were due to the inleakage of water.

WAP / P03095:12 Tank condition was normal.

WAP I P03095:13 Tank condition was normal. 


\begin{tabular}{|c|c|c|c|c|c|c|c|c|}
\hline \multirow{2}{*}{$\frac{\text { AREA }}{F}$} & \multirow{2}{*}{$\begin{array}{c}\begin{array}{c}\text { TANK OR } \\
\text { ANCILLARY }\end{array} \\
45\end{array}$} & \multicolumn{2}{|c|}{$\frac{\text { ACCESS OPENING }}{(A \text { OR I) }}$} & \multirow{2}{*}{$\frac{\text { DATE }}{09 / 05 / 03}$} & \multicolumn{3}{|c|}{$\frac{\text { INSPECTION METHOD }}{\text { IDENTIFICATION NUMBER }}$} & \multirow{2}{*}{$\begin{array}{l}\qquad \text { REMARKS } \\
\text { Inspection revealed a salt layer } \\
\text { throughout the tank with a few } \\
\text { small liquid pools visible. The reel } \\
\text { tape was positioned above liquid. } \\
\text { No unusual conditions were } \\
\text { observed. }\end{array}$} \\
\hline & & G & (I) & & CCTV & l & 1010 & \\
\hline $\mathrm{F}$ & 46 & LDB-01 & & $02 / 12 / 03$ & CCTV & l & 908 & $\begin{array}{l}\text { The conductivity probe was } \\
\text { properly deployed per setpoint } \\
\text { document J-JX-G-0001. }\end{array}$ \\
\hline $\mathrm{F}$ & 46 & LDB-01 & & 03/05/03 & CCTV & l & 908 & $\begin{array}{l}\text { The conductivity probe was } \\
\text { properly deployed per setpoint } \\
\text { document J-JX-G-0001. }\end{array}$ \\
\hline $\mathrm{F}$ & 46 & LDB-02 & & $01 / 22 / 03$ & CCTV & l & 908 & $\begin{array}{l}\text { The conductivity probe was } \\
\text { properly deployed per setpoint } \\
\text { document J-JX-G-0001. Liquid } \\
\text { was observed in the bottom of the } \\
\text { LDB. }\end{array}$ \\
\hline $\mathrm{F}$ & 46 & LDB-03 & & $01 / 22 / 03$ & CCTV & l & 908 & $\begin{array}{l}\text { The conductivity probe was } \\
\text { properly deployed per setpoint } \\
\text { document J-JX-G-0001. }\end{array}$ \\
\hline $\mathrm{F}$ & 46 & LDB-04 & & 03/05/03 & CCTV & l & 908 & $\begin{array}{l}\text { The conductivity probe was } \\
\text { properly deployed per setpoint } \\
\text { document J-JX-G-0001. }\end{array}$ \\
\hline $\mathrm{F}$ & 46 & $A-01$ & (A) & $02 / 25 / 03$ & WAP & l & P03096:01 & Tank condition was normal. \\
\hline $\mathrm{F}$ & 46 & A-02 & (A) & $02 / 25 / 03$ & CCTV & l & 926 & $\begin{array}{l}\text { The conductivity probe was } \\
\text { properly deployed per setpoint } \\
\text { document J-JX-G-0001. }\end{array}$ \\
\hline $\mathrm{F}$ & 46 & A-02 & (A) & $02 / 25 / 03$ & WAP & l & P03096:02 & Tank condition was normal. \\
\hline $\mathrm{F}$ & 46 & $\mathrm{~A}-03$ & (A) & $02 / 25 / 03$ & CCTV & l & 926 & $\begin{array}{l}\text { The conductivity probe was } \\
\text { properly deployed per setpoint } \\
\text { document J-JX-G-0001. }\end{array}$ \\
\hline $\mathrm{F}$ & 46 & A-03 & $(\mathrm{A})$ & 02/25/03 & WAP & l & P03096:03 & Tank condition was normal. \\
\hline $\mathrm{F}$ & 46 & A-04 & (A) & $02 / 25 / 03$ & CCTV & l & 926 & $\begin{array}{l}\text { The conductivity probe was } \\
\text { properly deployed per setpoint } \\
\text { document J-JX-G-0001. }\end{array}$ \\
\hline $\mathrm{F}$ & 46 & A-04 & $(\mathrm{A})$ & $02 / 25 / 03$ & WAP & l & P03096:04 & Tank condition was normal. \\
\hline $\mathrm{F}$ & 46 & $\mathrm{P}-01$ & (A) & $02 / 25 / 03$ & WAP & l & P03096:05 & Tank condition was normal. \\
\hline $\mathrm{F}$ & 46 & $\mathrm{P}-02$ & (A) & $02 / 25 / 03$ & WAP & l & P03096:06 & Tank condition was normal. \\
\hline $\mathrm{F}$ & 46 & $\mathrm{P}-03$ & (A) & $05 / 25 / 03$ & DP & l & P03117:01-27 & Tank condition was normal. \\
\hline
\end{tabular}




\begin{tabular}{|c|c|c|c|c|c|c|c|c|}
\hline \multirow{2}{*}{$\frac{\text { AREA }}{F}$} & \multirow{2}{*}{$\begin{array}{c}\text { TANK OR } \\
\text { ANCILLARY } \\
46\end{array}$} & \multicolumn{2}{|c|}{$\frac{\text { ACCESS OPENING }}{(A \text { OR I) }}$} & \multirow{2}{*}{$\frac{\text { DATE }}{05 / 25 / 03}$} & \multicolumn{3}{|c|}{$\frac{\text { INSPECTION METHOD }}{\text { IDENTIFICATION NUMBER }}$} & \multirow{2}{*}{$\begin{array}{l}\text { REMARKS } \\
\text { Tank condition was normal. } \\
\text { Stains on the ventilation duct were } \\
\text { due to the inleakage of water. }\end{array}$} \\
\hline & & P-04 & (A) & & DP & l & P03116:01-27 & \\
\hline $\mathrm{F}$ & 46 & P-05 & (A) & 05/25/03 & DP & l & P03115:01-28 & Tank condition was normal. \\
\hline $\mathrm{F}$ & 46 & P-06 & (A) & $05 / 25 / 03$ & DP & I & P03114:01-27 & Tank condition was normal. \\
\hline $\mathrm{F}$ & 46 & P-07 & (A) & $05 / 25 / 03$ & DP & l & P03113:01-27 & Tank condition was normal. \\
\hline $\mathrm{F}$ & 46 & P-08 & (A) & $02 / 25 / 03$ & WAP & l & P03096:07 & Tank condition was normal. \\
\hline $\mathrm{F}$ & 46 & P-09 & (A) & $02 / 25 / 03$ & WAP & l & P03096:08 & Tank condition was normal. \\
\hline $\mathrm{F}$ & 46 & $P-10$ & (A) & $02 / 25 / 03$ & WAP & l & P03096:09 & Tank condition was normal. \\
\hline $\mathrm{F}$ & 46 & P-11 & (A) & $02 / 25 / 03$ & WAP & l & P03096:10 & Tank condition was normal. \\
\hline $\mathrm{F}$ & 46 & $\mathrm{P}-12$ & (A) & $02 / 25 / 03$ & WAP & l & P03096:11 & Tank condition was normal. \\
\hline $\mathrm{F}$ & 46 & $P-13$ & (A) & $02 / 25 / 03$ & WAP & l & P03096:12 & Tank condition was normal. \\
\hline $\mathrm{F}$ & 46 & P-14 & (A) & $02 / 25 / 03$ & WAP & l & P03096:13 & Tank condition was normal. \\
\hline $\mathrm{F}$ & 46 & G & (I) & $01 / 31 / 03$ & CCTV & I & 905 & $\begin{array}{l}\text { Inspection documented the } \\
\text { conditions in the tank. An } \\
\text { irregular salt surface was } \\
\text { observed. }\end{array}$ \\
\hline $\mathrm{F}$ & 46 & G & (I) & 03/04/03 & CCTV & I & 905 & $\begin{array}{l}\text { Inspection documented the } \\
\text { conditions in the tank. A salt } \\
\text { mound was observed beneath the } \\
\text { BFV. }\end{array}$ \\
\hline$F$ & 46 & G & (I) & 03/21/03 & CCTV & I & 905 & $\begin{array}{l}\text { Inspection documented salt } \\
\text { formations in the tank. A large } \\
\text { mound was observed beneath the } \\
\text { BFV, and the cooling coils had salt } \\
\text { formations varying from } 1 \text { to } 4 \\
\text { inches thick. }\end{array}$ \\
\hline$F$ & 46 & G & (I) & $08 / 14 / 03$ & CCTV & I & 905 & $\begin{array}{l}\text { Inspection was made to verify } \\
\text { proper operation of the C-03 BFV } \\
\text { and perform a leak check. No } \\
\text { unusual conditions or leaks were } \\
\text { observed. }\end{array}$ \\
\hline
\end{tabular}




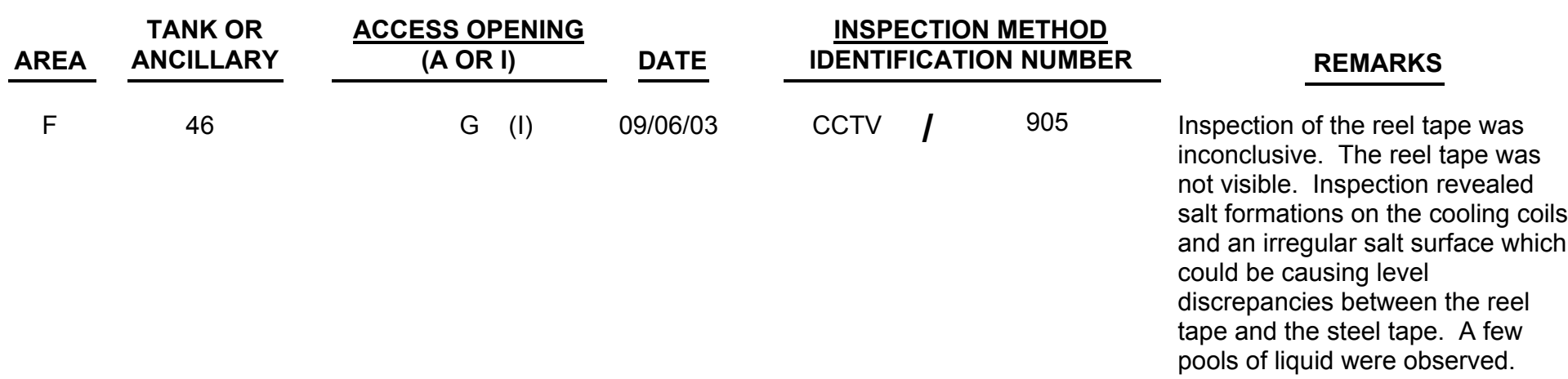
salt surface with some isolated pockets of liquid.

Inspection documented the conditions in the tank. A liquid surface was observed throughout the tank.

Inspection documented salt formations in the tank. No salt mounds were visible above the liquid level, and the cooling coils had approximately 3 inches of salt accumulation.

The conductivity probe was properly deployed per setpoint document J-JX-G-0001.

The conductivity probe was properly deployed per setpoint document J-JX-G-0001.

The conductivity probe was properly deployed per setpoint document J-JX-G-0001.

Tank condition was normal.

The conductivity probe was properly deployed per setpoint document J-JX-G-0001. 


\begin{tabular}{|c|c|c|c|c|c|c|c|c|}
\hline \multirow{2}{*}{$\frac{\text { AREA }}{F}$} & \multirow{2}{*}{$\begin{array}{c}\text { TANK OR } \\
\text { ANCILLARY } \\
47\end{array}$} & \multicolumn{2}{|c|}{$\frac{\text { ACCESS OPENING }}{(A \text { OR I) }}$} & \multirow{2}{*}{$\frac{\text { DATE }}{02 / 25 / 03}$} & \multicolumn{3}{|c|}{$\frac{\text { INSPECTION METHOD }}{\text { IDENTIFICATION NUMBER }}$} & \multirow{2}{*}{$\begin{array}{l}\qquad \text { REMARKS } \\
\qquad \begin{array}{l}\text { The conductivity probe was } \\
\text { properly deployed per setpoint } \\
\text { document J-JX-G-0001. }\end{array}\end{array}$} \\
\hline & & A-03 & (A) & & CCTV & l & 926 & \\
\hline $\mathrm{F}$ & 47 & A-03 & (A) & $02 / 25 / 03$ & WAP & l & P03097:03 & Tank condition was normal. \\
\hline $\mathrm{F}$ & 47 & A-04 & (A) & $02 / 25 / 03$ & CCTV & l & 926 & $\begin{array}{l}\text { The conductivity probe was } \\
\text { properly deployed per setpoint } \\
\text { document J-JX-G-0001. }\end{array}$ \\
\hline $\mathrm{F}$ & 47 & $\mathrm{~A}-04$ & (A) & $02 / 25 / 03$ & WAP & I & P03097:04 & Tank condition was normal. \\
\hline $\mathrm{F}$ & 47 & P-01 & (A) & $02 / 25 / 03$ & WAP & I & P03097:05 & Tank condition was normal. \\
\hline $\mathrm{F}$ & 47 & P-02 & (A) & $02 / 25 / 03$ & WAP & l & P03097:06 & Tank condition was normal. \\
\hline $\mathrm{F}$ & 47 & P-03 & (A) & $05 / 24 / 03$ & DP & l & P03118:01-28 & Tank condition was normal. \\
\hline $\mathrm{F}$ & 47 & P-04 & (A) & $05 / 24 / 03$ & DP & l & P03119:01-27 & Tank condition was normal. \\
\hline $\mathrm{F}$ & 47 & P-05 & $(\mathrm{A})$ & $05 / 24 / 03$ & DP & l & P03120:01-27 & Tank condition was normal. \\
\hline $\mathrm{F}$ & 47 & P-06 & (A) & $05 / 24 / 03$ & DP & l & P03121:01-28 & Tank condition was normal. \\
\hline $\mathrm{F}$ & 47 & P-07 & (A) & $05 / 24 / 03$ & DP & l & P03122:01-27 & Tank condition was normal. \\
\hline $\mathrm{F}$ & 47 & $\mathrm{P}-08$ & (A) & $02 / 25 / 03$ & WAP & l & P03097:07 & Tank condition was normal. \\
\hline $\mathrm{F}$ & 47 & P-09 & (A) & $02 / 25 / 03$ & WAP & l & P03097:08 & Tank condition was normal. \\
\hline $\mathrm{F}$ & 47 & $\mathrm{P}-10$ & (A) & $02 / 25 / 03$ & WAP & l & P03097:09 & Tank condition was normal. \\
\hline $\mathrm{F}$ & 47 & $\mathrm{P}-11$ & (A) & $02 / 25 / 03$ & WAP & l & P03097:10 & Tank condition was normal. \\
\hline $\mathrm{F}$ & 47 & $\mathrm{P}-12$ & (A) & $02 / 25 / 03$ & WAP & l & P03097:11 & Tank condition was normal. \\
\hline $\mathrm{F}$ & 47 & $P-13$ & (A) & $02 / 25 / 03$ & WAP & l & P03097:12 & Tank condition was normal. \\
\hline $\mathrm{F}$ & 47 & $\mathrm{P}-14$ & (A) & $02 / 25 / 03$ & WAP & l & P03097:13 & Tank condition was normal. \\
\hline $\mathrm{H}$ & 48 & $\begin{array}{r}\text { LDB Drain } \\
\text { Cell }\end{array}$ & & $04 / 02 / 03$ & CCTV & l & 908 & $\begin{array}{l}\text { The conductivity probe was } \\
\text { deployed at the setpoint. }\end{array}$ \\
\hline $\mathrm{H}$ & 48 & $A-01$ & (A) & 08/30/03 & WAP & l & P03187:01 & Tank condition was normal. \\
\hline $\mathrm{H}$ & 48 & A-02 & (A) & $08 / 30 / 03$ & CCTV & l & 926 & $\begin{array}{l}\text { The conductivity probe was } \\
\text { properly deployed per setpoint } \\
\text { document J-JX-G-0001. }\end{array}$ \\
\hline
\end{tabular}




\begin{tabular}{|c|c|c|c|c|c|c|c|c|}
\hline \multirow{2}{*}{$\frac{\text { AREA }}{H}$} & \multirow{2}{*}{$\begin{array}{c}\text { TANK OR } \\
\text { ANCILLARY } \\
48\end{array}$} & \multicolumn{2}{|c|}{$\frac{\text { ACCESS OPENING }}{(A \text { OR I) }}$} & \multirow{2}{*}{$\frac{\text { DATE }}{08 / 30 / 03}$} & \multicolumn{3}{|c|}{$\begin{array}{l}\text { INSPECTION METHOD } \\
\text { IDENTIFICATION NUMBER }\end{array}$} & \multirow{2}{*}{$\begin{array}{l}\text { REMARKS } \\
\text { Tank condition was normal. } \\
\text { Stains and marks on the } \\
\text { ventilation duct and annulus floor } \\
\text { had increased due to the } \\
\text { inleakage of water. }\end{array}$} \\
\hline & & A-02 & (A) & & WAP & l & P03187:02 & \\
\hline $\mathrm{H}$ & 48 & A-03 & (A) & $08 / 30 / 03$ & CCTV & l & 926 & $\begin{array}{l}\text { The conductivity probe was } \\
\text { properly deployed per setpoint } \\
\text { document J-JX-G-0001. }\end{array}$ \\
\hline $\mathrm{H}$ & 48 & A-03 & (A) & 08/30/03 & WAP & l & P03187:03 & Tank condition was normal. \\
\hline $\mathrm{H}$ & 48 & A-04 & (A) & $08 / 30 / 03$ & CCTV & l & 926 & $\begin{array}{l}\text { The conductivity probe was } \\
\text { properly deployed per setpoint } \\
\text { document J-JX-G-0001. }\end{array}$ \\
\hline $\mathrm{H}$ & 48 & $\mathrm{~A}-04$ & (A) & 08/30/03 & WAP & I & P03187:04 & Tank condition was normal. \\
\hline $\mathrm{H}$ & 48 & P-01 & (A) & 08/30/03 & WAP & I & P03187:05 & Tank condition was normal. \\
\hline $\mathrm{H}$ & 48 & $\mathrm{P}-02$ & (A) & 08/30/03 & WAP & I & P03187:06 & Tank condition was normal. \\
\hline $\mathrm{H}$ & 48 & P-03 & (A) & 08/30/03 & DP & l & P03180:01-27 & Tank condition was normal. \\
\hline $\mathrm{H}$ & 48 & $\mathrm{P}-04$ & (A) & 08/30/03 & DP & l & P03181:01-27 & $\begin{array}{l}\text { Tank condition was normal. } \\
\text { Stains and marks on the } \\
\text { secondary vessel wall, ventilation } \\
\text { duct and annulus floor were } \\
\text { caused by the inleakage of water. }\end{array}$ \\
\hline $\mathrm{H}$ & 48 & P-05 & (A) & 08/30/03 & DP & l & P03182:01-27 & $\begin{array}{l}\text { Tank condition was normal. } \\
\text { Stains and marks on the } \\
\text { secondary vessel wall, ventilation } \\
\text { duct and annulus floor were } \\
\text { caused by the inleakage of water. }\end{array}$ \\
\hline $\mathrm{H}$ & 48 & P-06 & (A) & 08/30/03 & WAP & l & P03187:07 & $\begin{array}{l}\text { Tank condition was normal. } \\
\text { Stains and marks on the } \\
\text { ventilation duct and annulus floor } \\
\text { had increased due to inleakage of } \\
\text { water. }\end{array}$ \\
\hline $\mathrm{H}$ & 48 & P-07 & (A) & 08/30/03 & WAP & l & P03187:08 & $\begin{array}{l}\text { Tank condition was normal. } \\
\text { Stains and marks on the } \\
\text { ventilation duct and annulus floor } \\
\text { had increased due to inleakage of } \\
\text { water. }\end{array}$ \\
\hline $\mathrm{H}$ & 48 & $\mathrm{P}-08$ & (A) & $08 / 30 / 03$ & WAP & l & P03187:09 & Tank condition was normal. \\
\hline $\mathrm{H}$ & 48 & P-09 & (A) & 08/30/03 & WAP & l & P03187:10 & Tank condition was normal. \\
\hline $\mathrm{H}$ & 48 & P-10 & $(\mathrm{A})$ & $08 / 30 / 03$ & DP & l & P03183:01-27 & Tank condition was normal. \\
\hline $\mathrm{H}$ & 48 & $\mathrm{P}-11$ & (A) & 08/30/03 & WAP & I & P03187:11 & Tank condition was normal. \\
\hline
\end{tabular}




\begin{tabular}{|c|c|c|c|c|c|c|c|c|}
\hline \multirow{2}{*}{$\frac{\text { AREA }}{\mathrm{H}}$} & \multirow{2}{*}{$\begin{array}{c}\text { TANK OR } \\
\text { ANCILLARY } \\
48\end{array}$} & \multicolumn{2}{|c|}{$\frac{\text { ACCESS OPENING }}{(A \text { OR I) }}$} & \multirow{2}{*}{$\frac{\text { DATE }}{08 / 30 / 03}$} & \multicolumn{3}{|c|}{$\frac{\text { INSPECTION METHOD }}{\text { IDENTIFICATION NUMBER }}$} & \multirow{2}{*}{$\frac{\text { REMARKS }}{\text { Tank condition was normal. }}$} \\
\hline & & $P-13$ & $(\mathrm{~A})$ & & WAP & l & P03187:12 & \\
\hline $\mathrm{H}$ & 48 & $\mathrm{P}-14$ & $(\mathrm{~A})$ & 08/30/03 & WAP & l & P03187:13 & Tank condition was normal. \\
\hline $\mathrm{H}$ & 49 & LDB-05 & & $09 / 25 / 03$ & CCTV & l & 1017 & $\begin{array}{l}\text { Inspection documented conditions } \\
\text { in the LDB. No obstructions were } \\
\text { observed at the vent or drain lines. }\end{array}$ \\
\hline $\mathrm{H}$ & 49 & $\begin{array}{r}\text { Underliner } \\
\text { Sump }\end{array}$ & & 09/17/03 & CCTV & l & 861 & $\begin{array}{l}\text { The conductivity probe was } \\
\text { properly deployed per setpoint } \\
\text { document J-JX-G-0001. Liquid } \\
\text { was observed in the LDB. }\end{array}$ \\
\hline $\mathrm{H}$ & 49 & A-01 & (A) & $08 / 31 / 03$ & WAP & l & P03184:01 & Tank condition was normal. \\
\hline $\mathrm{H}$ & 49 & A-02 & (A) & $08 / 31 / 03$ & WAP & l & P03184:04 & Tank condition was normal. \\
\hline $\mathrm{H}$ & 49 & A-02 & (A) & 09/01/03 & CCTV & l & 1008 & $\begin{array}{l}\text { The conductivity probe was } \\
\text { properly deployed per setpoint } \\
\text { document J-JX-G-0001. }\end{array}$ \\
\hline $\mathrm{H}$ & 49 & A-03 & (A) & $08 / 31 / 03$ & WAP & l & P03184:05 & Tank condition was normal. \\
\hline $\mathrm{H}$ & 49 & A-03 & $(\mathrm{A})$ & 09/01/03 & CCTV & l & 1008 & $\begin{array}{l}\text { The conductivity probe was } \\
\text { properly deployed per setpoint } \\
\text { document J-JX-G-0001. }\end{array}$ \\
\hline $\mathrm{H}$ & 49 & A-04 & $(\mathrm{A})$ & $08 / 31 / 03$ & WAP & l & P03184:10 & Tank condition was normal. \\
\hline $\mathrm{H}$ & 49 & A-04 & (A) & 09/01/03 & CCTV & l & 1008 & $\begin{array}{l}\text { The conductivity probe was } \\
\text { properly deployed per setpoint } \\
\text { document J-JX-G-0001. }\end{array}$ \\
\hline $\mathrm{H}$ & 49 & P-01 & $(\mathrm{A})$ & $08 / 31 / 03$ & WAP & l & P03184:02 & Tank condition was normal. \\
\hline $\mathrm{H}$ & 49 & P-02 & (A) & $08 / 31 / 03$ & WAP & l & P03184:03 & Tank condition was normal. \\
\hline $\mathrm{H}$ & 49 & P-03 & $(\mathrm{A})$ & $08 / 31 / 03$ & $\mathrm{DP}$ & l & P03165:01-27 & Tank condition was normal. \\
\hline $\mathrm{H}$ & 49 & P-04 & (A) & $08 / 31 / 03$ & DP & l & P03169:01-28 & Tank condition was normal. \\
\hline $\mathrm{H}$ & 49 & P-05 & (A) & $08 / 31 / 03$ & $\mathrm{DP}$ & l & P03166:01-27 & Tank condition was normal. \\
\hline $\mathrm{H}$ & 49 & P-06 & $(\mathrm{A})$ & $08 / 31 / 03$ & $\mathrm{DP}$ & l & P03167:01-27 & Tank condition was normal. \\
\hline $\mathrm{H}$ & 49 & P-07 & (A) & $08 / 31 / 03$ & DP & l & P03168:01-28 & Tank condition was normal. \\
\hline $\mathrm{H}$ & 49 & P-08 & (A) & $08 / 31 / 03$ & WAP & l & P03184:06 & Tank condition was normal. \\
\hline $\mathrm{H}$ & 49 & P-09 & $(\mathrm{A})$ & $08 / 31 / 03$ & WAP & l & P03184:07 & Tank condition was normal. \\
\hline
\end{tabular}




\begin{tabular}{|c|c|c|c|c|c|c|c|c|}
\hline \multirow{2}{*}{$\frac{\text { AREA }}{H}$} & \multirow{2}{*}{$\begin{array}{c}\begin{array}{c}\text { TANK OR } \\
\text { ANCILLARY }\end{array} \\
49\end{array}$} & \multicolumn{2}{|c|}{$\frac{\text { ACCESS OPENING }}{(A \text { OR I) }}$} & \multirow{2}{*}{$\frac{\text { DATE }}{08 / 31 / 03}$} & \multicolumn{3}{|c|}{$\begin{array}{l}\text { INSPECTION METHOD } \\
\text { IDENTIFICATION NUMBER }\end{array}$} & \multirow{2}{*}{$\frac{\text { REMARKS }}{\text { Tank condition was normal. }}$} \\
\hline & & $P-10$ & (A) & & WAP & l & P03184:08 & \\
\hline $\mathrm{H}$ & 49 & $P-11$ & (A) & $08 / 31 / 03$ & WAP & l & P03184:09 & Tank condition was normal. \\
\hline $\mathrm{H}$ & 49 & $\mathrm{P}-12$ & (A) & $08 / 31 / 03$ & WAP & l & P03184:12 & Tank condition was normal. \\
\hline $\mathrm{H}$ & 49 & $P-13$ & (A) & $08 / 31 / 03$ & WAP & l & P03184:13 & Tank condition was normal. \\
\hline $\mathrm{H}$ & 49 & $\mathrm{P}-14$ & (A) & $08 / 31 / 03$ & WAP & l & P03184:11 & Tank condition was normal. \\
\hline $\mathrm{H}$ & 49 & B-04 & (I) & $07 / 12 / 03$ & CCTV & l & 861 & $\begin{array}{l}\text { Inspection verified the position of } \\
\text { the HLLCP after installation. The } \\
\text { HLLCP was properly deployed, } \\
\text { and no obstructions were } \\
\text { observed. }\end{array}$ \\
\hline $\mathrm{H}$ & 49 & B-05 & (I) & 06/26/03 & CCTV & l & 861 & $\begin{array}{l}\text { Inspection documented riser } \\
\text { equipment configuration and tank } \\
\text { contents. The waste was covered } \\
\text { with a thin salt crust with a few } \\
\text { isolated areas of liquid. }\end{array}$ \\
\hline
\end{tabular}

CCTV was used to document salt sounding and thickness of salt layer on the liquid surface.

Inspection documented conditions beneath the riser. A flange with c-clamp (dustcover) was observed on the nozzle. The nitrogen nozzle extends beneath the riser and turns above the nozzle box.

CCTV was used to document salt sounding and thickness of salt layer on the liquid surface.

The conductivity probe was properly deployed per setpoint document J-JX-G-0001.

The conductivity probe was properly deployed per setpoint document J-JX-G-0001.

CCTV was used to leak check valve WTS-V-274. A leak was observed at the Gra-Loc connector.

CCTV was used to leak check valve WTS-V-274. A leak was observed at the Gra-Loc connector. 


\begin{tabular}{|c|c|c|c|c|c|c|c|}
\hline \multirow{2}{*}{$\frac{\text { AREA }}{\mathrm{H}}$} & \multirow{2}{*}{$\begin{array}{c}\begin{array}{c}\text { TANK OR } \\
\text { ANCILLARY }\end{array} \\
50\end{array}$} & $\frac{\text { ACCESS OPENING }}{(A \text { OR I) }}$ & \multirow{2}{*}{$\frac{\text { DATE }}{03 / 05 / 03}$} & \multicolumn{3}{|c|}{$\frac{\text { INSPECTION METHOD }}{\text { IDENTIFICATION NUMBER }}$} & \multirow{2}{*}{$\begin{array}{l}\qquad \text { REMARKS } \\
\qquad \text { CCTV was used to leak check } \\
\text { valve WTS-V-274. No leaks were } \\
\text { observed. }\end{array}$} \\
\hline & & VB & & CCTV & l & 929 & \\
\hline $\mathrm{H}$ & 50 & VB & $03 / 05 / 03$ & CCTV & l & 929 & $\begin{array}{l}\text { CCTV was used to leak check } \\
\text { valve WTS-V-274. No leaks were } \\
\text { observed. }\end{array}$ \\
\hline $\mathrm{H}$ & 50 & VB & $03 / 12 / 03$ & CCTV & l & 929 & $\begin{array}{l}\text { CCTV was used to leak check } \\
\text { Gra-Loc connector on the HDB- } 07 \\
\text { side of valve WTS-V-276. No } \\
\text { leaks were observed. }\end{array}$ \\
\hline $\mathrm{H}$ & 50 & $A-01 \quad(A)$ & $08 / 31 / 03$ & WAP & l & P03186:01 & Tank condition was normal. \\
\hline $\mathrm{H}$ & 50 & $A-02 \quad(A)$ & $08 / 31 / 03$ & WAP & l & P03186:04 & Tank condition was normal. \\
\hline $\mathrm{H}$ & 50 & A-02 (A) & 09/01/03 & CCTV & l & 1008 & $\begin{array}{l}\text { The conductivity probe was } \\
\text { properly deployed per setpoint } \\
\text { document J-JX-G-0001. }\end{array}$ \\
\hline $\mathrm{H}$ & 50 & A-03 (A) & $08 / 31 / 03$ & WAP & l & P03186:05 & Tank condition was normal. \\
\hline $\mathrm{H}$ & 50 & $\mathrm{~A}-03 \quad(\mathrm{~A})$ & 09/01/03 & CCTV & l & 1008 & $\begin{array}{l}\text { The conductivity probe was } \\
\text { properly deployed per setpoint } \\
\text { document J-JX-G-0001. }\end{array}$ \\
\hline $\mathrm{H}$ & 50 & A-04 (A) & $08 / 31 / 03$ & WAP & l & P03186:10 & Tank condition was normal. \\
\hline $\mathrm{H}$ & 50 & $\mathrm{~A}-04 \quad(\mathrm{~A})$ & 09/01/03 & CCTV & l & 1008 & $\begin{array}{l}\text { The conductivity probe was } \\
\text { properly deployed per setpoint } \\
\text { document J-JX-G-0001. }\end{array}$ \\
\hline $\mathrm{H}$ & 50 & P-01 (A) & 08/31/03 & WAP & I & P03186:02 & Tank condition was normal. \\
\hline $\mathrm{H}$ & 50 & $\mathrm{P}-02 \quad(\mathrm{~A})$ & 08/31/03 & WAP & I & P03186:03 & Tank condition was normal. \\
\hline $\mathrm{H}$ & 50 & $\mathrm{P}-03 \quad(\mathrm{~A})$ & $08 / 31 / 03$ & $\mathrm{DP}$ & I & P03175:01-27 & Tank condition was normal. \\
\hline $\mathrm{H}$ & 50 & $\mathrm{P}-04 \quad(\mathrm{~A})$ & $08 / 31 / 03$ & $\mathrm{DP}$ & l & P03176:01-27 & Tank condition was normal. \\
\hline $\mathrm{H}$ & 50 & P-05 (A) & $08 / 31 / 03$ & $\mathrm{DP}$ & l & P03177:01-28 & Tank condition was normal. \\
\hline $\mathrm{H}$ & 50 & P-06 (A) & $08 / 31 / 03$ & $\mathrm{DP}$ & l & P03178:01-27 & Tank condition was normal. \\
\hline $\mathrm{H}$ & 50 & P-07 (A) & $08 / 31 / 03$ & $\mathrm{DP}$ & I & P03179:01-27 & Tank condition was normal. \\
\hline $\mathrm{H}$ & 50 & P-08 (A) & $08 / 31 / 03$ & WAP & l & P03186:06 & Tank condition was normal. \\
\hline $\mathrm{H}$ & 50 & P-09 (A) & $08 / 31 / 03$ & WAP & l & P03186:07 & Tank condition was normal. \\
\hline $\mathrm{H}$ & 50 & $P-10 \quad(A)$ & $08 / 31 / 03$ & WAP & I & P03186:08 & Tank condition was normal. \\
\hline
\end{tabular}




\begin{tabular}{|c|c|c|c|c|c|c|c|c|}
\hline \multirow{2}{*}{$\frac{\text { AREA }}{H}$} & \multirow{2}{*}{$\begin{array}{c}\begin{array}{c}\text { TANK OR } \\
\text { ANCILLARY }\end{array} \\
50\end{array}$} & \multicolumn{2}{|c|}{$\frac{\text { ACCESS OPENING }}{(A \text { OR I) }}$} & \multirow{2}{*}{$\frac{\text { DATE }}{08 / 31 / 03}$} & \multicolumn{3}{|c|}{$\begin{array}{l}\text { INSPECTION METHOD } \\
\text { IDENTIFICATION NUMBER }\end{array}$} & \multirow{2}{*}{$\frac{\text { REMARKS }}{\text { Tank condition was normal. }}$} \\
\hline & & $\mathrm{P}-11$ & (A) & & WAP & l & P03186:09 & \\
\hline $\mathrm{H}$ & 50 & $\mathrm{P}-12$ & (A) & $08 / 31 / 03$ & WAP & l & P03186:11 & Tank condition was normal. \\
\hline $\mathrm{H}$ & 50 & $P-13$ & (A) & $08 / 31 / 03$ & WAP & l & P03186:12 & Tank condition was normal. \\
\hline $\mathrm{H}$ & 50 & $\mathrm{P}-14$ & (A) & $08 / 31 / 03$ & WAP & l & P03186:13 & Tank condition was normal. \\
\hline $\mathrm{H}$ & 50 & B-04 & (I) & $01 / 14 / 03$ & CCTV & l & 885 & $\begin{array}{l}\text { Inspection was performed to } \\
\text { document any obstructions that } \\
\text { may exist beneath the riser. No } \\
\text { obstructions were observed. }\end{array}$ \\
\hline $\mathrm{H}$ & 50 & B-05 & (I) & $12 / 22 / 03$ & CCTV & l & 883 & $\begin{array}{l}\text { Inspection was made to } \\
\text { investigate conductivity probe } \\
\text { alarm. A leak was observed at the } \\
\text { discharge nozzle. The nozzle was } \\
\text { tightened; however, a small leak } \\
\text { was still observed. After the } \\
\text { nozzle was loosened and } \\
\text { retightened, no leaks were } \\
\text { observed. }\end{array}$ \\
\hline $\mathrm{H}$ & 50 & G & (I) & 05/08/03 & CCTV & l & 958 & $\begin{array}{l}\text { CCTV was used to monitor } \\
\text { surface agitation during slurry } \\
\text { pump operations. Cooling coil } \\
\text { loop \#12 had excessive } \\
\text { movement indicating that the coil } \\
\text { may have become separated from } \\
\text { the supports at the bottom. }\end{array}$ \\
\hline $\mathrm{H}$ & 50 & G & (I) & $05 / 13 / 03$ & CCTV & l & 958 & $\begin{array}{l}\text { Inspection documented surface } \\
\text { conditions. The surface of the } \\
\text { waste was covered with a thin film, } \\
\text { and exposed solids were observed } \\
\text { at the south wall. }\end{array}$ \\
\hline $\mathrm{H}$ & 50 & G & (I) & $05 / 14 / 03$ & CCTV & l & 958 & $\begin{array}{l}\text { Inspection documented tank } \\
\text { conditions and the mound of } \\
\text { solids observed near the south } \\
\text { wall. }\end{array}$ \\
\hline $\mathrm{H}$ & 50 & G & (I) & $05 / 15 / 03$ & CCTV & I & 958 & $\begin{array}{l}\text { CCTV was used to document tank } \\
\text { conditions and index slurry } \\
\text { pumps. }\end{array}$ \\
\hline $\mathrm{H}$ & 50 & G & (I) & $05 / 16 / 03$ & CCTV & I & 958 & $\begin{array}{l}\text { Inspection documented tank } \\
\text { conditions and the mound of } \\
\text { solids observed near the south } \\
\text { wall. }\end{array}$ \\
\hline $\mathrm{H}$ & 50 & G & (I) & 05/19/03 & CCTV & l & 958 & $\begin{array}{l}\text { Inspection documented tank } \\
\text { contents and observed solids } \\
\text { exposed near the south wall. }\end{array}$ \\
\hline $\mathrm{H}$ & 50 & G & (I) & 05/20/03 & CCTV & I & 958 & $\begin{array}{l}\text { Inspection documented tank } \\
\text { conditions. }\end{array}$ \\
\hline
\end{tabular}




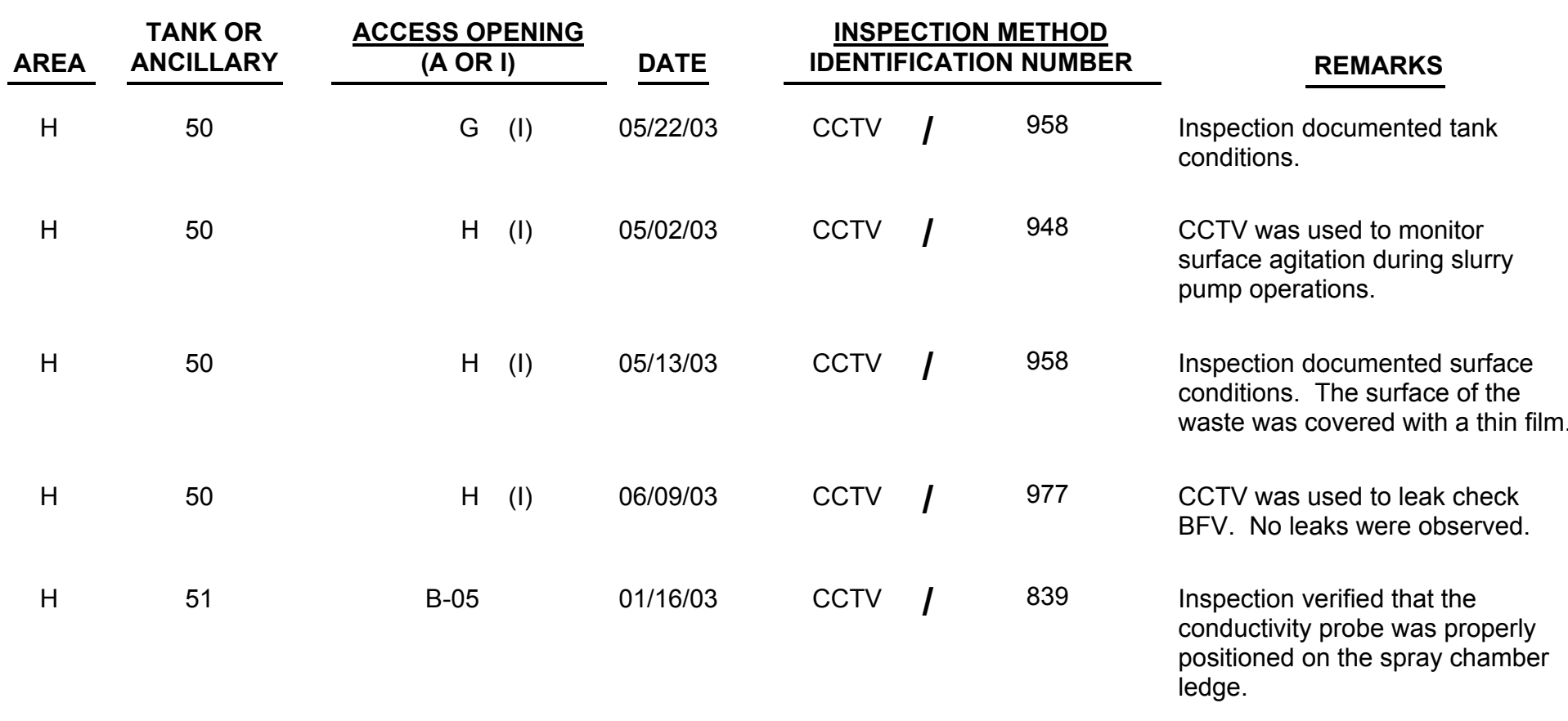

Inspection verified that the conductivity probe was properly positioned on the spray chamber ledge.

CCTV was used to leak check TTJ. No leaks were observed.

Inspection verified the position of the conductivity probe in the spray chamber. The conductivity probe was positioned horizontally on the riser ledge below the TTJ plate.

The conductivity probe was resting on the lower ledge of the spray chamber. It was properly positioned.

CCTV was used to leak check jet nozzles. No leaks were observed.

Conductivity probe WTS-LE-2024 was observed suspended above the valve box bottom. It is approximately 0.25 to 0.5 inches above the bottom of the valve box.

CCTV was used to inspect the steam line to the jet via valve WTS-V-73. Approximately 7.5 feet towards the gang valve house and 35 feet towards the jet were inspected. No obstructions were observed; however, liquid was encountered approximately 33 feet towards the jet indicating that the line was still plugged. 


\begin{tabular}{|c|c|c|c|c|}
\hline AREA & $\begin{array}{l}\text { TANK OR } \\
\text { ANCILLARY }\end{array}$ & $\frac{\text { ACCESS OPENING }}{(A \text { OR I) }}$ & DATE & $\frac{\text { INSPECTION METHOD }}{\text { IDENTIFICATION NUMBER }}$ \\
\hline $\mathrm{H}$ & 51 & LDB-01 & 01/19/03 & CCTV \\
\hline
\end{tabular}

\section{REMARKS}

CCTV was used to document conditions in LDB after an alarm was received. The probe was visible beneath the standpipe with no unusual conditions observed.

$02 / 13 / 03$

$\mathrm{H}$

51

Valve Box

$02 / 13 / 03$

$04 / 22 / 03$

$\mathrm{H}$

VB

51

A-01 (A) 10/07/03

$03 / 21 / 03$

A-02 (A)

$\mathrm{A}-02 \quad(\mathrm{~A})$

(A)

$08 / 29 / 03$

A-03

(A)

08/29/03

A-03 (A)

08/30/03

$\mathrm{H}$

$\mathrm{H}$

51

$\mathrm{H}$

51

$\begin{array}{lll}\text { A-04 } & \text { (A) } & 08 / 29 / 03 \\ \text { A-04 (A) } & 08 / 30 / 03\end{array}$

P-01

(A)

08/29/03
CCTV I

839

CCTV I

839

CCTV I

951

WAP

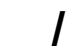

P03215:01

CCTV

926

WAP

P03185:11 is contacting the floor of the valve box.

Conductivity probe WTS-LE-2029 is contacting the floor of the valve box.

Inspection revealed no liquid in the valve box. The conductivity probe was positioned just above the floor. A piece of plastic and a flashlight were observed in the valve box.

Tank condition was normal. Stains and marks on the annulus floor beneath the P-01 riser were caused by the inleakage of water.

The conductivity probe was properly deployed per setpoint document J-JX-G-0001.

Tank condition was normal.

Tank condition was normal.

The conductivity probe was properly deployed per setpoint document J-JX-G-0001.

WAP I P03185:02 Tank condition was normal.

CCTV I $926 \quad$ The conductivity probe was properly deployed per setpoint document J-JX-G-0001.

Tank condition was normal. Stains on the primary and secondary vessel walls, annulus floor and ventilation duct were due to inleakage of water.

Tank condition was normal. Stains on the annulus floor and ventilation duct were due to inleakage of water.
Conductivity probe WTS-LE-2028 


\begin{tabular}{|c|c|c|c|c|c|c|c|c|}
\hline \multirow{2}{*}{$\frac{\text { AREA }}{H}$} & \multirow{2}{*}{$\begin{array}{c}\begin{array}{c}\text { TANK OR } \\
\text { ANCILLARY }\end{array} \\
51\end{array}$} & \multicolumn{2}{|c|}{$\frac{\text { ACCESS OPENING }}{(\text { A OR I) }}$} & \multirow{2}{*}{$\frac{\text { DATE }}{08 / 30 / 03}$} & \multicolumn{3}{|c|}{$\frac{\text { INSPECTION METHOD }}{\text { IDENTIFICATION NUMBER }}$} & \multirow{2}{*}{$\frac{\text { REMARKS }}{\text { Tank condition was normal. }}$} \\
\hline & & P-03 & (A) & & DP & I & P03170:01-27 & \\
\hline $\mathrm{H}$ & 51 & P-04 & (A) & $08 / 30 / 03$ & DP & I & P03171:01-27 & Tank condition was normal. \\
\hline $\mathrm{H}$ & 51 & P-05 & (A) & $08 / 30 / 03$ & DP & I & P03172:01-27 & Tank condition was normal. \\
\hline $\mathrm{H}$ & 51 & P-06 & (A) & $08 / 30 / 03$ & DP & I & P03173:01-26 & Tank condition was normal. \\
\hline $\mathrm{H}$ & 51 & P-07 & (A) & $08 / 30 / 03$ & DP & I & P03174:01-27 & Tank condition was normal. \\
\hline $\mathrm{H}$ & 51 & P-08 & (A) & $08 / 29 / 03$ & WAP & I & P03185:08 & Tank condition was normal. \\
\hline $\mathrm{H}$ & 51 & P-09 & (A) & $08 / 29 / 03$ & WAP & I & P03185:09 & Tank condition was normal. \\
\hline $\mathrm{H}$ & 51 & $P-10$ & (A) & $08 / 29 / 03$ & WAP & I & P03185:10 & Tank condition was normal. \\
\hline $\mathrm{H}$ & 51 & $P-11$ & (A) & $08 / 29 / 03$ & WAP & I & P03185:03 & $\begin{array}{l}\text { Tank condition was normal. } \\
\text { Chromate deposits on the tank } \\
\text { wall from a previous cooling coil } \\
\text { leak had dried and fallen off of the } \\
\text { wall. }\end{array}$ \\
\hline $\mathrm{H}$ & 51 & $\mathrm{P}-12$ & (A) & $08 / 29 / 03$ & WAP & I & P03185:04 & Tank condition was normal. \\
\hline $\mathrm{H}$ & 51 & $P-13$ & (A) & $08 / 29 / 03$ & WAP & I & P03185:05 & Tank condition was normal. \\
\hline $\mathrm{H}$ & 51 & $P-14$ & (A) & $08 / 29 / 03$ & WAP & I & P03185:06 & Tank condition was normal. \\
\hline $\mathrm{H}$ & 51 & B-03 & (I) & $03 / 11 / 03$ & CCTV & 1 & 931 & $\begin{array}{l}\text { The HLLCP was suspended in the } \\
\text { tank with no obstructions } \\
\text { observed beneath the probe. }\end{array}$ \\
\hline $\mathrm{H}$ & 51 & B-03 & (I) & $06 / 19 / 03$ & CCTV & I & 954 & $\begin{array}{l}\text { Inspection of the tank interior } \\
\text { revealed liquid throughout the } \\
\text { tank. No unusual conditions were } \\
\text { observed. }\end{array}$ \\
\hline $\mathrm{H}$ & 51 & $V-01$ & (I) & 03/19/03 & CCTV & l & 931 & $\begin{array}{l}\text { CCTV was used to monitor TTJ } \\
\text { during transfer activities. Steam } \\
\text { was observed leaking from the } \\
\text { stuffing box on the steam leg of } \\
\text { the jet. }\end{array}$ \\
\hline $\mathrm{H}$ & 51 & $V-01$ & (I) & $03 / 23 / 03$ & CCTV & 1 & 931 & $\begin{array}{l}\text { CCTV was used to monitor TTJ } \\
\text { during transfer activities. Steam } \\
\text { was observed leaking from the } \\
\text { stuffing box on the steam leg of } \\
\text { the jet. }\end{array}$ \\
\hline $\mathrm{H}$ & 51 & $V-01$ & (I) & 09/18/03 & CCTV & I & 954 & $\begin{array}{l}\text { CCTV was used to leak check } \\
\text { connector in V-01 riser. Liquid } \\
\text { was observed leaking from wall } \\
\text { connection below valve } \\
\text { WTS-V- } 287 \text {. }\end{array}$ \\
\hline
\end{tabular}




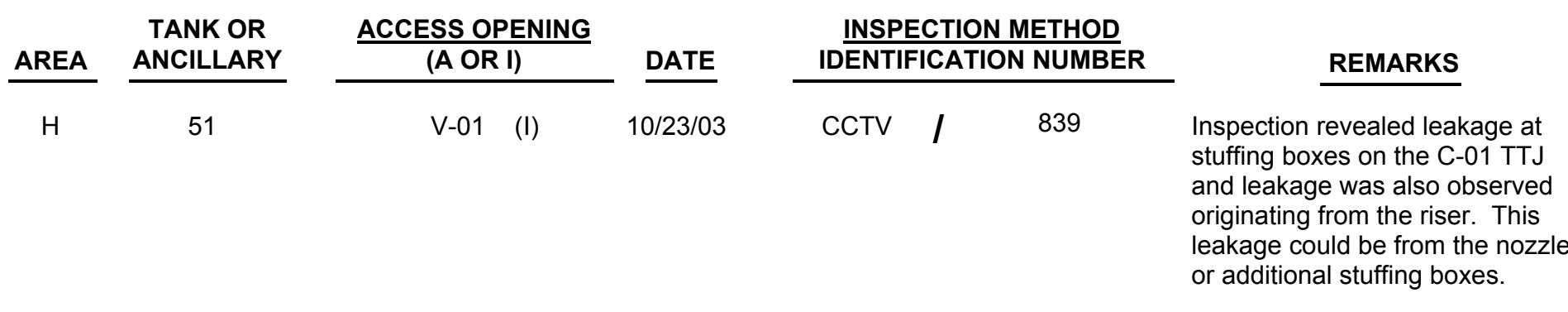

$\mathrm{H}$

$\mathrm{H}$

51

V-01

(I)

$12 / 29 / 03$

CCTV /

1051

$\mathrm{H}$

CCWS

$\mathrm{H}$

CCWS

$\mathrm{F}$

CTS

$\mathrm{F}$

CTS

SW

SE

09/03/03

CCTV /

812

05/05/03

HELIUM /

HE-03-008

954

CCTV was used to leak check WTS-V-293. Leakage was observed from the flange.

CCTV was used to leak check DWPF inhibited water connector. A leak was observed at the Gra-lock connection.

Helium tracer tests of the CCWS for Tanks 9 - 16 identified a leaksite southeast of Tank 12.

Helium tracer tests of the CCWS for Tanks 9 - 16 identified leaksites southeast of Tank 12 and west of Tank 13.

Inspection documented conditions in the 242-3F CTS pit to aid in planning for closure. The floor was dry beneath the riser with accumulations of water around the tank increasing in depth towards the sump in the southwest corner. The underside of the cell covers was in good condition. The stainless steel liner was buckled near the bottom of the cell. Abandoned jumpers and equipment were observed throughout the cell.

Inspection documented conditions in the 242-3F CTS pit to aid in planning for closure. Clear water was observed on the cell floor overflowing the sump and covering approximately half of the cell floor. The stainless steel liner was buckled near the bottom of the cell. The underside of the cell covers was in good condition. Abandoned jumpers were observed in the cell on top of installed jumpers, the tank and on the floor. A dummy connector was observed on the floor, and a hard hat was observed in the sump. 


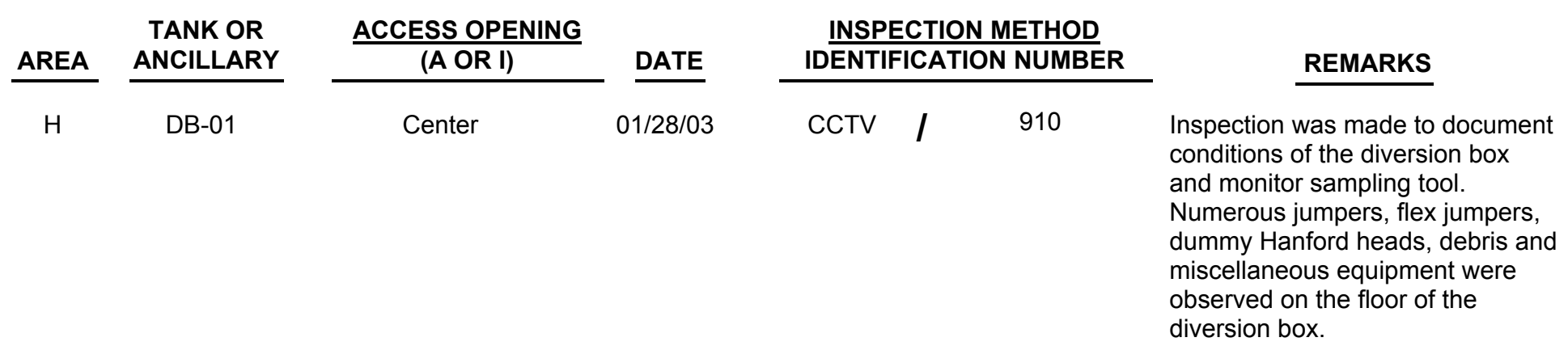

$\mathrm{F}$

F

DB-02

DB-02

$\mathrm{F}$

$\mathrm{F}$

DB-02

MLDB-02

$\mathrm{F}$

DB-02

MLDB-03

$12 / 22 / 03$

CCTV /

964

F

DB-02

MLDB-05

04/09/03

CCTV

908

$\mathrm{F}$

DB-02

MLDB-05

$05 / 19 / 03$

CCTV

964

F

DB-02

MLDB-05

12/15/03

CCTV

964

F

DB-02

MLDB-08

05/15/03

CCTV

964

F

DB-02
Sump
10/23/03
964

964

964

964

(1)




\begin{tabular}{|c|c|c|c|c|}
\hline AREA & $\begin{array}{l}\text { TANK OR } \\
\text { ANCILLARY }\end{array}$ & $\frac{\text { ACCESS OPENING }}{(A \text { OR I) }}$ & DATE & $\frac{\text { INSPECTION METHOD }}{\text { IDENTIFICATION NUMBER }}$ \\
\hline $\mathrm{H}$ & DB-02 & & $10 / 14 / 03$ & CCTV \\
\hline
\end{tabular}

\section{REMARKS}

CCTV was used to document condition of transfer line segment 930 from HDB-2 to Tank 11. A small amount of dried waste remained in the line.

$\mathrm{H}$

DB-02

$10 / 14 / 03$

CCTV I

922

CCTV was used to document condition of transfer line segment 665 from HDB-2 to Tank 12. A small amount of dried waste remained in the line.

$\mathrm{H}$

DB-02

LDB-01

07/12/03

CCTV

964

The conductivity probe was properly deployed per setpoint document J-JX-G-0001.

$\mathrm{H}$

DB-02

LDB-01

$12 / 15 / 03$

CCTV I

964

The conductivity probe was properly deployed per setpoint document J-JX-G-0001.

$\mathrm{H}$

DB-02

MLDB-03

09/12/03

CCTV

964

Inspection documented conditions in the MLDB. An abandoned conductivity probe and $1 / 2$ to $3 / 4$ inches of mud/liquid were observed at the bottom. Surface corrosion and shallow pitting were observed on the walls of the MLDB.

$\mathrm{H}$

DB-02

South

$02 / 14 / 03$

CCTV I

922

$\mathrm{H}$

DB-02

South

03/06/03

CCTV

922

$\mathrm{H}$

DB-02

South

$03 / 14 / 03$

CCTV

922

$\mathrm{H}$

DB-02

South

04/09/03

CCTV I

922

Inspection revealed that no obstructions would prevent the sump conductivity probe from being properly installed.

The conductivity probe was observed contacting the floor of the diversion box. Paint chips and chips of concrete from the bottom of the cell covers were observed on the floor of the diversion box.

Inspection documented the position of the conductivity probe. The conductivity probe was repositioned and appeared to be contacting the floor of the diversion box.

Inspection documented position of the conductivity probe. The conductivity probe was suspended several inches above the floor of the diversion box.

Inspection verified that no liquid was present on the floor of the diversion box and the conductivity probe was properly deployed. 


\begin{tabular}{|c|c|c|c|c|c|c|c|}
\hline \multirow{2}{*}{$\frac{\text { AREA }}{\mathrm{H}}$} & \multirow{2}{*}{$\begin{array}{c}\begin{array}{c}\text { TANK OR } \\
\text { ANCILLARY }\end{array} \\
\text { DB-02 }\end{array}$} & \multirow{2}{*}{$\frac{\frac{\text { ACCESS OPENING }}{\text { (A OR I) }}}{\text { South }}$} & \multirow{2}{*}{$\frac{\text { DATE }}{06 / 13 / 03}$} & \multicolumn{3}{|c|}{$\frac{\text { INSPECTION METHOD }}{\text { IDENTIFICATION NUMBER }}$} & \multirow{2}{*}{$\begin{array}{l}\text { REMARKS } \\
\text { Inspection verified that no liquid } \\
\text { was present on the floor of the } \\
\text { diversion box and the conductivity } \\
\text { probe was properly deployed. }\end{array}$} \\
\hline & & & & CCTV & I & 922 & \\
\hline $\mathrm{H}$ & DB-02 & South & $07 / 12 / 03$ & CCTV & I & 922 & $\begin{array}{l}\text { Inspection documented conditions } \\
\text { beneath the conductivity probe. } \\
\text { No unusual conditions were } \\
\text { observed. }\end{array}$ \\
\hline $\mathrm{H}$ & DB-02 & South & 08/10/03 & CCTV & I & 922 & $\begin{array}{l}\text { Inspection verified that no liquid } \\
\text { was present on the floor of the } \\
\text { diversion box and the conductivity } \\
\text { probe was properly deployed. }\end{array}$ \\
\hline $\mathrm{H}$ & DB-02 & South & 09/08/03 & CCTV & I & 922 & $\begin{array}{l}\text { Inspection verified that no liquid } \\
\text { was present on the floor of the } \\
\text { diversion box and the conductivity } \\
\text { probe was properly deployed. }\end{array}$ \\
\hline $\mathrm{H}$ & DB-02 & South & $10 / 04 / 03$ & CCTV & I & 922 & $\begin{array}{l}\text { Inspection verified that no liquid } \\
\text { was present on the floor of the } \\
\text { diversion box and the conductivity } \\
\text { probe was properly deployed. }\end{array}$ \\
\hline
\end{tabular}

Inspection was made to document conditions beneath the conductivity probe and verify that no liquid was present. The floor of the diversion box was damp with no standing liquid observed. No unusual conditions were observed.

Inspection was made to document conditions beneath the conductivity probe and verified that no liquid was present. The floor of the diversion box was damp with no standing liquid observed. No unusual conditions were observed.

Inspection documented conditions beneath the conductivity probe. The floor was damp; however, no standing liquid was observed. No unusual conditions were observed.

;

DB-03

MLDB-01

04/02/03

CCTV I

908 


\begin{tabular}{|c|c|c|c|c|}
\hline AREA & $\begin{array}{l}\text { TANK OR } \\
\text { ANCILLARY }\end{array}$ & $\frac{\text { ACCESS OPENING }}{(\text { A OR I) }}$ & DATE & $\frac{\text { INSPECTION METHOD }}{\text { IDENTIFICATION NUMBER }}$ \\
\hline $\mathrm{F}$ & DB-03 & MLDB-01 & 07/05/03 & CCTV \\
\hline
\end{tabular}

\section{REMARKS}

The conductivity probe was properly deployed per setpoint document J-JX-G-0001.

Approximately $1 / 4$ inch of standing water and an absorbent cloth were observed in the bottom of the

07/17/03 CCTV / 964

$03 / 20 / 03$

CCTV / 908

MLDB-02

$03 / 20 / 03$

$10 / 23 / 03$

CCTV

1030

Sump

03/30/03

CCTV /

941

$\mathrm{H}$

DB-03

DB-04

09/02/03

CCTV

1009

F

F

DB-04

09/05/03

CCTV

NA

09/12/03

CCTV

1009

F

DB-04

$\mathrm{F}$

DB-04

10/17/03

CCTV

1026

F

DB-04

10/18/03

CCTV

1026

10/25/03
CCTV
MLDB.

The conductivity probe was document J-JX-G-0001.

The conductivity probe was properly deployed per setpoint document J-JX-G-0001.

Inspection verified that no liquid was present in the sump.

Inspection documented two pipe wrenches, a dummy connector head, and a jumper on the floor of the diversion box. Cables and a steel plate were in the sump.

Verified valve WTS-V-225 in the closed position. The valve does not close completely.

Verified valve WTS-V-225 in the closed position. The valve did not close completely.

Verified valve WTS-V-225 closed. The top stop was misaligned and prevented the valve from closing completely.

CCTV was used to perform leak check of nozzles and jumpers. Leaks were observed on wall nozzle 8 , nozzle $16 \mathrm{D}$ on jumper 12-13D-16D and valve WTS-V-224 on jumper 10,11-5.

CCTV was used to perform leak check of nozzles and jumpers. A leak was observed on valve WTS-V-224 on jumper 10,11-5.

CCTV was used to leak check of nozzles and jumpers. A leak was observed on valve WTS-V-224. properly deployed per setpoint 


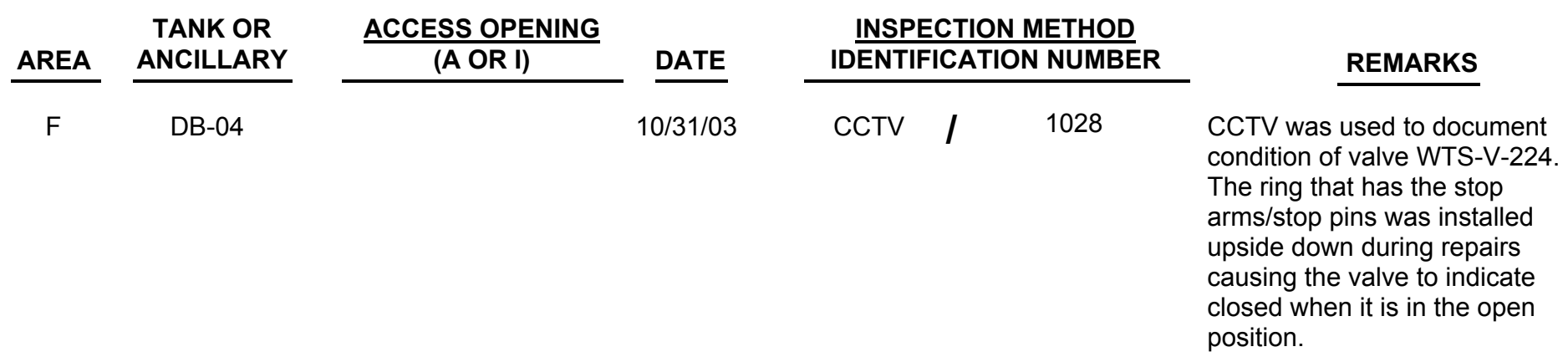

LDB-03

02/26/03

CCTV

908

LDB-04

04/08/03

CCTV

908

LDB-05

02/26/03

CCTV

908

LDB-06

09/03/03

CCTV

964

F

DB-04

LDB-07

$02 / 12 / 03$

CCTV

908

DB-04

LDB-07

03/05/03

CCTV

908

F

DB-04

LDB-08

09/26/03

CCTV

964

DB-04

LDB-13

02/25/03

CCTV /

908

LDB-13

04/28/03

CCTV

908 properly deployed per setpoint document J-JX-G-0001.

The conductivity probe was properly deployed per setpoint document J-JX-G-0001.

CCTV was used to leak check nozzles and verify valve positions. No leaks were observed on nozzles 8, 5A, 5B, 5C, 13D, and 16D. Valves WTS-V-223, 224, 225, 226, 229, 230, 231, 232, 233, $234,235,236,237$, and 309 were in the closed position.

The conductivity probe was properly deployed per setpoint document J-JX-G-0001.

The conductivity probe was properly deployed per setpoint document J-JX-G-0001.

The conductivity probe was properly deployed per setpoint document J-JX-G-0001.

The conductivity probe was properly deployed per setpoint document J-JX-G-0001.

The conductivity probe was properly deployed per setpoint document J-JX-G-0001.

CCTV was used to document position of conductivity probe in response to an alarm. The conductivity probe was not visible due to liquid, mud and debris around the standpipe.

The conductivity probe was properly deployed per setpoint document J-JX-G-0001. 


\begin{tabular}{|c|c|c|c|c|}
\hline AREA & $\begin{array}{l}\text { TANK OR } \\
\text { ANCILLARY }\end{array}$ & $\frac{\text { ACCESS OPENING }}{(A \text { OR I) }}$ & DATE & $\frac{\text { INSPECTION METHOD }}{\text { IDENTIFICATION NUMBER }}$ \\
\hline $\mathrm{F}$ & DB-04 & Open Cell & 10/09/03 & CCTV \\
\hline
\end{tabular}

\section{REMARKS}

CCTV was used to monitor removal of jumpers 7-9(FDB4)15A and 4-6(FDB4)13D-13 and document conditions of wall nozzles and floor nozzles. Wall nozzle 8 and floor nozzles $5 A, 5 B$, $5 \mathrm{C}, 2,4$, and 6 had gasket material remaining on the face and required cleaning. Hard hats, dummy connectors, a jumper, sand bag, tags, plastic and concrete from core drilling were observed on the cell floor.

$11 / 17 / 03$

CCTV

1041

$\mathrm{H}$

DB-04

$$
\text { DB-04 }
$$

$11 / 18 / 03$

CCTV

1041

$$
\mathrm{H}
$$

$\mathrm{H}$

DB-04

$11 / 19 / 03$

CCTV

1041

$\mathrm{H}$

DB-04

LDB-06

$11 / 24 / 03$

CCTV /

873

$\mathrm{H}$

DB-05

Sump

$08 / 24 / 03$

CCTV /

1001

$\mathrm{H}$

DB-05

Sump

$08 / 28 / 03$

CCTV I

1005

$\mathrm{H}$

DB-05
08/30/03
CCTV I
CCTV was used to perform leak check during flush of $3 \mathrm{H}$ Evaporator to Tank 32 transfer line through HDB-04. No leaks were observed.

Inspection was performed to locate source of liquid on the floor and increase in the sump. The source of the leakage was not located.

Inspection determined that valves WTS-V-39 and 540 and the connector head on the B2 nozzle were leaking. Dummy connector heads, a flashlight and other extraneous items were observed.

The conductivity probe was properly deployed per setpoint document J-JX-G-0001.

CCTV was used to document conditions in the sump. A small amount of liquid was observed in the sump.

Inspection of the conductivity probe standpipe revealed liquid. Some debris was observed on the sump bottom. No obstructions or unusual conditions were observed.

Inspection documented conditions in the diversion box. Some extraneous items were observed on the floor, and approximately 1 -2 inches of liquid was observed in the sump. No unusual conditions conditions were observed. 


\begin{tabular}{|c|c|c|c|c|c|c|}
\hline \multirow{2}{*}{$\frac{\text { AREA }}{\mathrm{H}}$} & \multirow{2}{*}{$\begin{array}{c}\text { TANK OR } \\
\text { ANCILLARY } \\
\text { DB-06 }\end{array}$} & $\frac{\text { ACCESS OPENING }}{(\mathrm{A} \text { OR I) }}$ & \multirow{2}{*}{$\frac{\text { DATE }}{03 / 03 / 03}$} & \multicolumn{2}{|c|}{$\frac{\text { INSPECTION METHOD }}{\text { IDENTIFICATION NUMBER }}$} & REMARKS \\
\hline & & & & CCTV & 928 & $\begin{array}{l}\text { Inspection documented } \\
\text { manipulation of valve WTS-V- } 61 \text {. } \\
\text { The upper and lower valve stops } \\
\text { were broken allowing the valve to } \\
\text { rotate } 360 \text { degrees. }\end{array}$ \\
\hline
\end{tabular}

$\mathrm{H}$

DB-06

$\mathrm{H}$

DB-06

$\mathrm{H}$

DB-06

$\mathrm{H}$

DB-06

$\mathrm{H}$

DB-06

$\mathrm{H}$

DB-06

LDB-01

LDB-01

06/03/03

CCTV

928

03/04/03

CCTV

928

CCTV / $\quad 928$

03/04/03

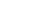

CCTV was used to observe valve WTS-V-61 for leaks during Tank 37 to Tank 32 transfer. No leaks were observed.

Verified valve WTS-V-61 in the open position. Verified valve WTS-V-55 in the closed position. Moisture was observed on the floor of the diversion box and on valve WTS-V-61. Salt build up was observed on valve WTS-V-55.

Inspection revealed that valve WTS-V-61 was leaking at the valve body. Liquid was observed on the floor, and dried waste was observed on the valve body.

Inspection documented leak check of valve WTS-V-61. The valve was leaking at the valve body.

Inspection verified no leaks at connector heads $6,7,1,9 \mathrm{~A}$ and 14.

$06 / 12 / 03$

CCTV

980

The conductivity probe was properly deployed per setpoint document J-JX-G-0001.

The conductivity probe was properly deployed per setpoint document J-JX-G-0001.

$\mathrm{H}$

DB-06

LDB-02

03/09/03

CCTV

908

$\mathrm{H}$

DB-06

LDB-03

03/10/03

CCTV

908

$\mathrm{H}$

DB-06

LDB-04

03/10/03

CCTV

908

$\mathrm{H}$

DB-06

LDB-05

02/27/03

CCTV / 908
The conductivity probe was properly deployed per setpoint document J-JX-G-0001.

The conductivity probe was properly deployed per setpoint document J-JX-G-0001.

The conductivity probe was properly deployed per setpoint document J-JX-G-0001.

The conductivity probe was properly deployed per setpoint document J-JX-G-0001. 


\begin{tabular}{|c|c|c|c|c|c|c|c|}
\hline \multirow{2}{*}{$\frac{\text { AREA }}{\mathrm{H}}$} & \multirow{2}{*}{$\begin{array}{c}\text { TANK OR } \\
\text { ANCILLARY } \\
\text { DB-06 }\end{array}$} & \multirow{2}{*}{$\begin{array}{l}\frac{\text { ACCESS OPENING }}{\text { (A OR I) }} \\
\text { LDB-05 }\end{array}$} & \multirow{2}{*}{$\frac{\text { DATE }}{03 / 09 / 03}$} & \multicolumn{3}{|c|}{$\begin{array}{l}\text { INSPECTION METHOD } \\
\text { IDENTIFICATION NUMBER }\end{array}$} & \multirow{2}{*}{$\begin{array}{l}\text { REMARKS } \\
\text { The conductivity probe was } \\
\text { properly deployed per setpoint } \\
\text { document J-JX-G-0001. }\end{array}$} \\
\hline & & & & CCTV & I & 908 & \\
\hline $\mathrm{H}$ & DB-06 & LDB-05 & 03/29/03 & CCTV & I & 908 & $\begin{array}{l}\text { The conductivity probe was } \\
\text { properly deployed per setpoint } \\
\text { document J-JX-G-0001. }\end{array}$ \\
\hline $\mathrm{H}$ & DB-07 & & 03/03/03 & CCTV & I & 927 & $\begin{array}{l}\text { CCTV was used to leak check } \\
\text { jumper } 21,25(\mathrm{HDB} 7) 5 \mathrm{E} \text {. No } \\
\text { leaks were observed on jumper } \\
21,25(\mathrm{HDB} 7) 5 \mathrm{E} ; \text { however, a leak } \\
\text { was observed at the 5D connector } \\
\text { head. }\end{array}$ \\
\hline $\mathrm{H}$ & DB-07 & & 04/11/03 & CCTV & I & 932 & $\begin{array}{l}\text { Inspection verified that the flush } \\
\text { water line end cap was welded. }\end{array}$ \\
\hline $\mathrm{H}$ & DB-07 & & 05/20/03 & CCTV & I & 965 & $\begin{array}{l}\text { The condition of the stainless steel } \\
\text { liner walls was normal. Some } \\
\text { insignificant voids were observed } \\
\text { at the top of the concrete vault. } \\
\text { These voids were caused by the } \\
\text { removal and installation of the } \\
\text { concrete cell covers. No unusual } \\
\text { conditions were observed on the } \\
\text { nozzle faces. }\end{array}$ \\
\hline
\end{tabular}

H $\quad$ DB-07

05/26/03

CCTV / 971

CCTV was used to perform leak check of connector heads. A leak was observed on connector head 12.

CCTV was used to tighten connector heads $5 \mathrm{~A}$ and 12 and perform leak check. A leak was observed on connector head $5 A$.

CCTV was used to facilitate remote loosening of connector head $5 A$ on jumper 12-5A. Two of the three pins that hold the jaws in place fell off of the connector head.

CCTV was used to leak check connector heads $12,5 \mathrm{~A}, 13,6 \mathrm{~B}$, $14 \mathrm{~A}, 8 \mathrm{~A}, 5 \mathrm{~B}, 6 \mathrm{~A}, 7 \mathrm{~A}$ and $17 \mathrm{~A}$. No leaks were observed.

The conductivity probe was properly deployed per setpoint document J-JX-G-0001. 


\begin{tabular}{|c|c|c|c|c|c|c|c|}
\hline \multirow{2}{*}{$\frac{\text { AREA }}{H}$} & \multirow{2}{*}{$\begin{array}{c}\begin{array}{c}\text { TANK OR } \\
\text { ANCILLARY }\end{array} \\
\text { DB-07 }\end{array}$} & \multirow{2}{*}{$\frac{\frac{\text { ACCESS OPENING }}{(\text { A OR I) }}}{\text { LDB-02 }}$} & \multirow{2}{*}{$\frac{\text { DATE }}{04 / 24 / 03}$} & \multicolumn{3}{|c|}{$\begin{array}{l}\text { INSPECTION METHOD } \\
\text { IDENTIFICATION NUMBER }\end{array}$} & \multirow{2}{*}{$\begin{array}{l}\qquad \text { REMARKS } \\
\text { The conductivity probe was } \\
\text { properly deployed per setpoint } \\
\text { document J-JX-G-0001. }\end{array}$} \\
\hline & & & & CCTV & I & 908 & \\
\hline $\mathrm{H}$ & DB-07 & LDB-02 & $10 / 13 / 03$ & CCTV & I & 964 & $\begin{array}{l}\text { The conductivity probe was } \\
\text { properly deployed per setpoint } \\
\text { document J-JX-G-0001. }\end{array}$ \\
\hline $\mathrm{H}$ & DB-07 & LDB-03 & $01 / 23 / 03$ & CCTV & I & 908 & $\begin{array}{l}\text { The conductivity probe was } \\
\text { properly deployed per setpoint } \\
\text { document J-JX-G-0001. }\end{array}$ \\
\hline $\mathrm{H}$ & DB-07 & LDB-03 & $07 / 15 / 03$ & CCTV & I & 873 & $\begin{array}{l}\text { The conductivity probe was } \\
\text { properly deployed per setpoint } \\
\text { document J-JX-G-0001. }\end{array}$ \\
\hline $\mathrm{H}$ & DB-07 & LDB-04 & $04 / 24 / 03$ & CCTV & I & 908 & $\begin{array}{l}\text { The conductivity probe was } \\
\text { properly deployed per setpoint } \\
\text { document J-JX-G-0001. }\end{array}$ \\
\hline
\end{tabular}

The conductivity probe was properly deployed per setpoint document J-JX-G-0001.

The conductivity probe was properly deployed per setpoint document J-JX-G-0001.

The conductivity probe was properly deployed per setpoint document J-JX-G-0001.

The conductivity probe was properly deployed per setpoint document J-JX-G-0001.

The conductivity probe was properly deployed per setpoint document J-JX-G-0001.

The conductivity probe was properly deployed per setpoint document J-JX-G-0001.

The conductivity probe was properly deployed per setpoint document J-JX-G-0001.

The conductivity probe was properly deployed per setpoint document J-JX-G-0001.

CCTV was used to leak check connector heads 21, 5E and 5D. No leaks were observed. 


\begin{tabular}{|c|c|c|c|c|c|c|c|}
\hline$\frac{\text { AREA }}{F}$ & $\begin{array}{c}\begin{array}{c}\text { TANK OR } \\
\text { ANCILLARY }\end{array} \\
\text { EVAP-01 }\end{array}$ & \multirow{2}{*}{$\begin{array}{c}\text { ACCESS OPENING } \\
\text { (A OR I) } \\
\begin{array}{c}\text { North } \\
\text { Overhead } \\
\text { Tank }\end{array}\end{array}$} & $\frac{\text { DATE }}{11 / 05 / 03}$ & \multicolumn{3}{|c|}{$\frac{\text { INSPECTION METHOD }}{\text { IDENTIFICATION NUMBER }}$} & \multirow{2}{*}{$\begin{array}{l}\text { REMARKS } \\
\text { CCTV revealed the tank wall and } \\
\text { floor were free of deposits. A thin } \\
\text { layer }(<2 \text { inches }) \text { of solids was } \\
\text { observed on the floor beneath the } \\
\text { water level of } 7 \text { inches. }\end{array}$} \\
\hline $\mathrm{F}$ & EVAP-01 & & $11 / 05 / 03$ & CCTV & I & 1036 & \\
\hline
\end{tabular}

F EVAP-01

$\mathrm{F}$

$$
\text { EVAP-01 }
$$

$\mathrm{F}$

$$
\text { EVAP-01 }
$$

Receiver
Cell

North

Overhead

Tank

Receiver

Cell

$12 / 16 / 03$

CCTV I

1036

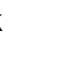




\begin{tabular}{|c|c|c|c|c|}
\hline AREA & $\begin{array}{l}\text { TANK OR } \\
\text { ANCILLARY }\end{array}$ & $\frac{\text { ACCESS OPENING }}{(A \text { OR I) }}$ & DATE & $\frac{\text { INSPECTION METHOD }}{\text { IDENTIFICATION NUMBER }}$ \\
\hline $\mathrm{F}$ & EVAP-16 & SE & 10/24/03 & CCTV \\
\hline
\end{tabular}

\section{REMARKS}

Inspection documented the condition of the evaporator cell, pot exterior, jumpers and associated piping. Stains observed around the E-3 nozzle appear to be dried salt. It is unlikely to have leaked from E-3, the feed jumper, which has a dip leg extending 10 feet into the evaporator pot. The adjacent E-4 nozzle was used as camera port for a pot inspection in May 2003. Flushing during camera removal is the likely cause of the stains. The area will be inspected in January 2004 to monitor for changes. All other areas observed were normal.
F

F

$\mathrm{H}$

EVAP-16

Condenser

Cell

$\mathrm{H}$

EVAP-16

Condenser

Cell

Sump
01/14/03

CCTV

904

$12 / 02 / 03$

CCTV

963

$03 / 12 / 03$

CCTV /

893

$03 / 28 / 03$

CCTV /

893
Inspection was made to document conditions of the vent pipe and thermocouple. No unusual conditions were observed.

Inspection was made to document cell conditions and verify that no leakage existed after an increase in radiation levels was detected. No leakage was observed.

Inspection documented conditions of the condenser cell. No degradation of the cell walls was observed. Ladders, plastic, mercury and paint flakes were observed on the cell floor. Liquid continues to leak from the top flange of the condenser. Mild surface corrosion was observed on the condenser supports.

CCTV was used to facilitate tightening of the upper flange bolts and document cell conditions. Mild surface corrosion was observed on the condenser, condenser supports and some service piping. Stains observed on the cell wall appeared to be from inleakage. A small amount of mercury and extraneous items were observed on the cell floor.

Inspection documented leakage at the upper flange after the flange bolts were tightened. 


\begin{tabular}{|c|c|c|c|c|c|c|c|}
\hline \multirow{2}{*}{$\frac{\text { AREA }}{\mathrm{H}}$} & \multirow{2}{*}{$\begin{array}{c}\text { TANK OR } \\
\text { ANCILLARY } \\
\text { EVAP-16 }\end{array}$} & \multirow{2}{*}{$\begin{array}{c}\frac{\text { ACCESS OPENING }}{\text { (A OR I) }} \\
\begin{array}{c}\text { Condenser } \\
\text { Cell }\end{array}\end{array}$} & \multirow{2}{*}{$\frac{\text { DATE }}{07 / 06 / 03}$} & \multicolumn{3}{|c|}{$\frac{\text { INSPECTION METHOD }}{\text { IDENTIFICATION NUMBER }}$} & \multirow{2}{*}{$\begin{array}{l}\qquad \text { REMARKS } \\
\text { CCTV was used to leak check } \\
\text { flanges in the condenser cell. No } \\
\text { leaks were observed. }\end{array}$} \\
\hline & & & & CCTV & I & 984 & \\
\hline $\mathrm{H}$ & EVAP-16 & NE & 06/09/03 & CCTV & I & 943 & $\begin{array}{l}\text { CCTV was used to monitor jumper } \\
\text { N6(2H)N9 for leaks during flush } \\
\text { from Tank } 50 \text {. No leaks were } \\
\text { observed. }\end{array}$ \\
\hline $\mathrm{H}$ & EVAP-16 & NE & $11 / 20 / 03$ & CCTV & I & 1042 & $\begin{array}{l}\text { Inspection documented conditions } \\
\text { of the evaporator cell walls, pot } \\
\text { exterior, service piping, cell covers } \\
\text { and jumpers. No unusual } \\
\text { conditions were observed. Hard } \\
\text { hats, mop handles, dummy } \\
\text { connector heads and other } \\
\text { extraneous items were observed } \\
\text { on the cell floor. }\end{array}$ \\
\hline
\end{tabular}

CCTV was used to document conditions in the cell. No unusual conditions were observed. Stains and deposits on the stainless steel liner were caused by the inleakage of rainwater.

H EVAP-16

Overheads Tank \#1

03/27/03

CCTV /

893

Inspection documented the conditions in the tank and facilitated flushing activities. A small amount of mercury and several wire ties were observed on the bottom of the tank.

Inspection documented the conditions in the tank and facilitated flushing activities. A small amount of mercury was observed on the bottom of the tank.

Inspection documented conditions of the evaporator cell walls, pot exterior, service piping, cell covers and jumpers. No unusual conditions were observed. Hard hats, mop handles, dummy connector heads and other extraneous items were observed on the cell floor. A strip of plastic and cell cover gasket material were observed in the sump. 


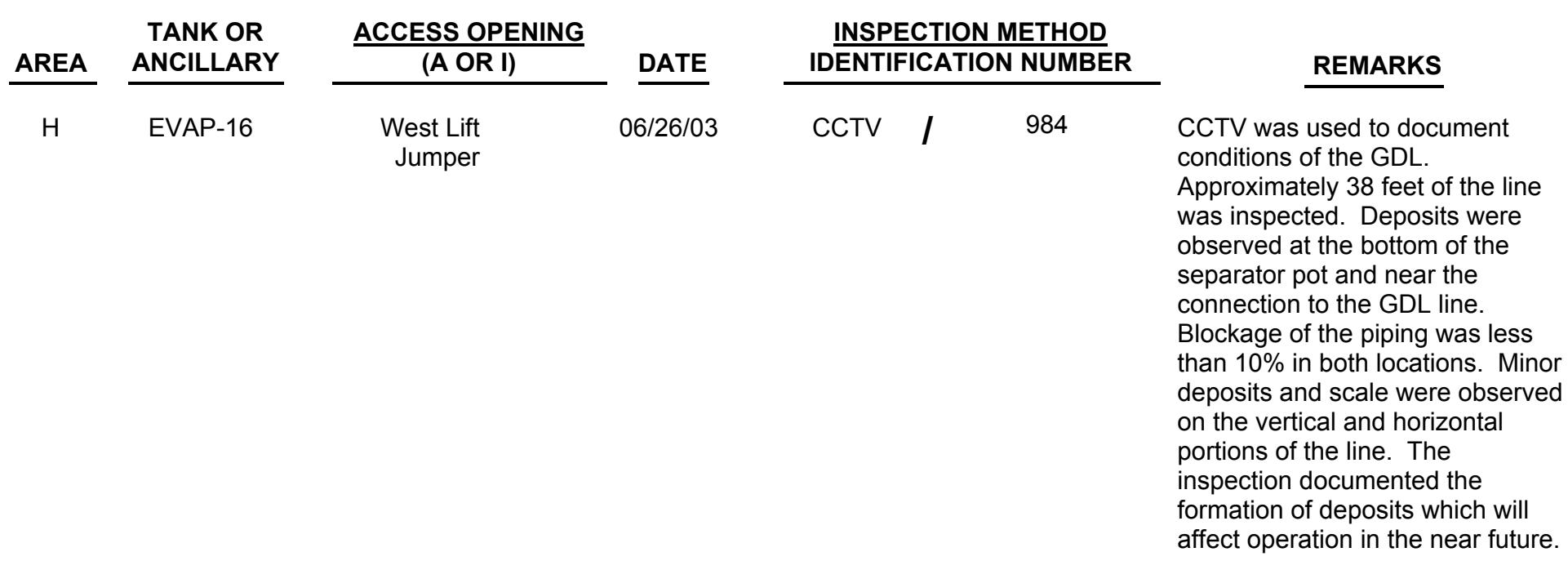

CCTV was used to document conditions of the lift line. The entire lift line was inspected. Inspection showed deposits forming on the piping walls. The piping at the connection to the lift jumper had deposits blocking approximately $10 \%$ of the pipe diameter. Approximately 2 inches of solids were observed at the bottom of the cone. Several rocklike deposits formed a small "rubble pile" about 1 inch below the lift inlet. The inspection documented the formation of deposits which will affect operation in the near future.

Inspection of the separator pot after cleaning revealed only minor deposits in the pot. The GDL had a thin coating covering the entire pipe wall.

Inspection documented the

conditions of the vessel. A light scale and fine, loose solids were observed on the vessel walls. Loose solids had settled at the bottom of the vessel.

Approximately 5.5 feet of liquid remained in the vessel. A light

scale was also observed on the tube bundle and service piping. 


\begin{tabular}{|c|c|c|c|c|c|c|}
\hline AREA & $\begin{array}{c}\text { TANK OR } \\
\text { ANCILLARY }\end{array}$ & $\frac{\text { ACCESS OPENING }}{(A \text { OR I) }}$ & DATE & IDENT & $\frac{\text { ETHOD }}{\text { NUMBER }}$ & REMARKS \\
\hline$F$ & EVAP-16 & E-04 (I) & 05/14/03 & CCTV & 963 & $\begin{array}{l}\text { Inspection documented conditions } \\
\text { of the evaporator pot. No } \\
\text { significant changes were observed } \\
\text { on the tube bundle, warming coil, } \\
\text { thermowells, exposed piping or } \\
\text { pot interior. Approximately } 4 \text { - } 6 \\
\text { inches of liquid remained in the } \\
\text { pot, and there appeared to be less } \\
\text { than } 4 \text { inches of solids in the } \\
\text { bottom of the vessel. Stains were } \\
\text { visible just above the operating } \\
\text { level of } 104 \text { inches with no } \\
\text { apparent changes in the upper } \\
\text { regions of the vessel. No solids or } \\
\text { deposits were observed on the } \\
\text { bottom of the demister. A light } \\
\text { scale was observed on the tube } \\
\text { bundle and vessel walls. The } \\
\text { thermowells, dip tubes, vessel } \\
\text { sidewalls, coil supports, stay rods } \\
\text { and other exposed surfaces were } \\
\text { free of any solids or deposits. The } \\
\text { intake of the lift line was not } \\
\text { visible. All observations were } \\
\text { consistent with normal evaporator } \\
\text { operation. }\end{array}$ \\
\hline
\end{tabular}

Inspection documented conditions of the pot. No large areas of deposits had formed since last inspection. Several areas of deposits were observed on the vessel wall near the feed jumper inlet line and are $<0.25$ inches. A thin scale was observed on the pot walls, tube bundle, warming coil and service piping. Small amounts of loose solids had settled on piping beneath the operating level. The bottom of the cone and the lift line inlet were not visible due to the amount of remaining liquid. The bottom support collar for the warming coils was visible through the liquid. Observations in this inspection were consistent with normal evaporator operation, and no significant changes were noted since last inspection on 12/7/02. 


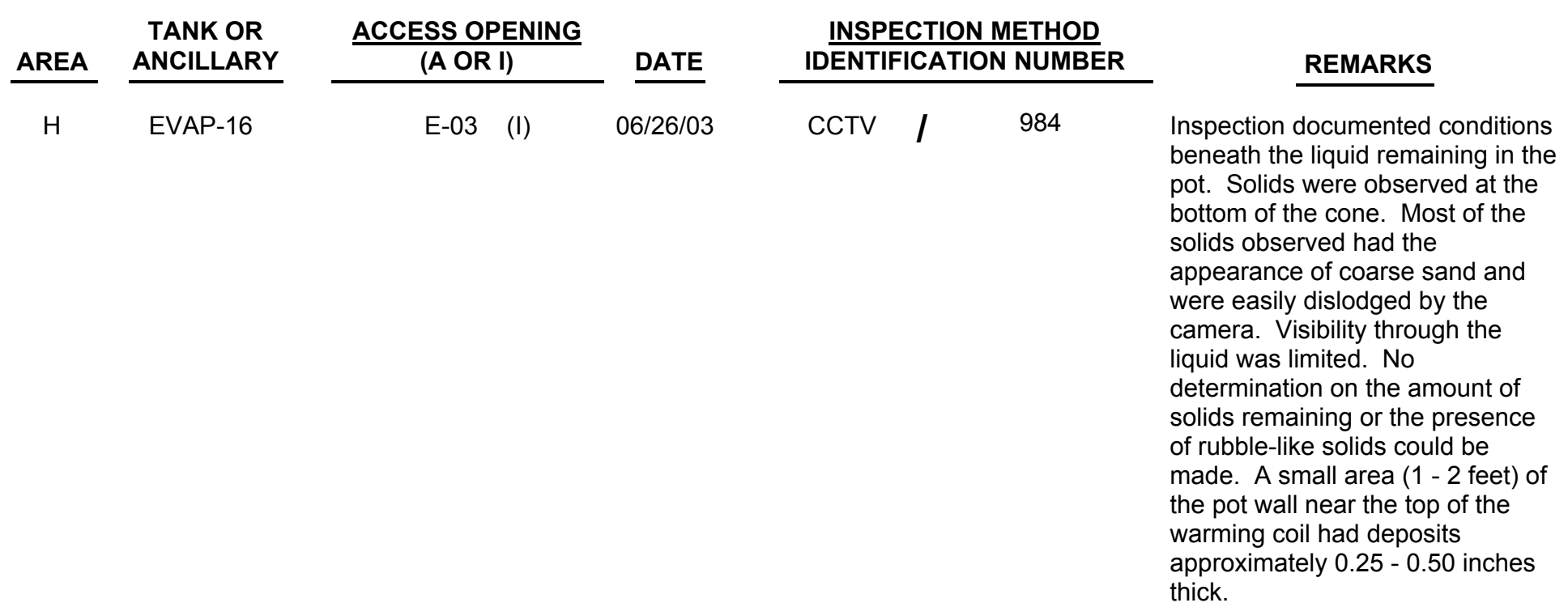

Inspection documented conditions of the pot. No significant increase in solids or deposits was observed since inspection in 6/03; however, the formation of deposits had increased since inspected in $7 / 02$. Thin scale and deposits up to $1 / 4 "$ thick were observed on the vessel walls. The tube bundle was covered with scale approximately $1 / 32$ " to $3 / 32$ " thick, and the support braces were covered with scale approximately $1 / 32$ " thick. All observable service piping was coated with both hardened deposits and loose solids. The warming coil had loose solids on top and deposits attached to the sides in several places. The support braces were covered with scale approximately $1 / 32$ " thick.

Inspection documented conditions beneath the liquid remaining in the pot. Solids were observed at the bottom of the vessel. The solids appeared to be fine loose, flakes/chips or small pebble sized. They appeared to be hardened and not easily dislodged. Approximately $11 / 2$ inches of solids were resting on the bottom of the cone. A box cutter was observed resting on top of the material at the bottom of the cone. The box cutter was removed on 9/26/03. 


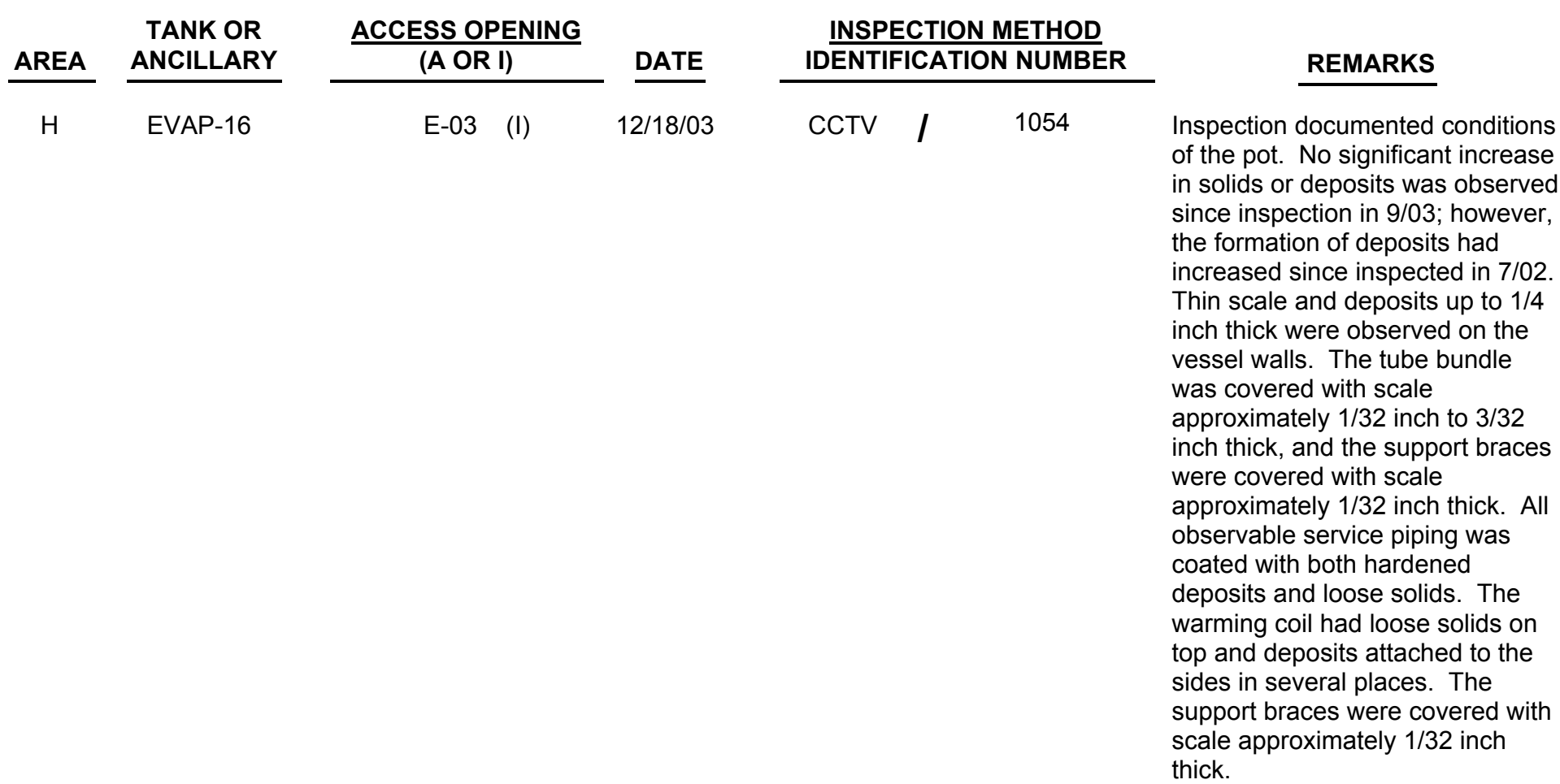

Inspection verified that the condenser vent line was free of obstructions.

CCTV was used to facilitate the installation of the separator pot drain jumper and perform leak check. No leaks were observed.

CCTV was used to leak check the S6 flange. No leaks were observed.

GDL Cell

$11 / 11 / 03$

CCTV /

1039

Inspection documented conditions of the GDL cell, cell covers, and piping. No unusual conditions were observed. 


\begin{tabular}{|c|c|c|c|c|c|c|c|}
\hline \multirow{2}{*}{$\frac{\text { AREA }}{\mathrm{H}}$} & \multirow{2}{*}{$\begin{array}{c}\text { TANK OR } \\
\text { ANCILLARY } \\
\text { EVAP-25 }\end{array}$} & \multirow{2}{*}{$\begin{array}{c}\frac{\text { ACCESS OPENING }}{\text { (A OR I) }} \\
\text { NE }\end{array}$} & \multirow{2}{*}{$\frac{\text { DATE }}{02 / 26 / 03}$} & \multicolumn{3}{|c|}{$\frac{\text { INSPECTION METHOD }}{\text { IDENTIFICATION NUMBER }}$} & \multirow{2}{*}{$\begin{array}{l}\text { REMARKS } \\
\text { CCTV was used to observe for } \\
\text { leakage at the U2 nozzle. No } \\
\text { leaks were observed. }\end{array}$} \\
\hline & & & & CCTV & I & 903 & \\
\hline $\mathrm{H}$ & EVAP-25 & NE & $11 / 12 / 03$ & CCTV & I & 1039 & $\begin{array}{l}\text { Inspection documented conditions } \\
\text { of the evaporator cell, pot exterior, } \\
\text { service piping, cell covers and } \\
\text { jumpers. No unusual conditions } \\
\text { were observed. }\end{array}$ \\
\hline $\mathrm{H}$ & EVAP-25 & $\begin{array}{r}\text { Overhead } \\
\text { Tank \#1 }\end{array}$ & $01 / 19 / 03$ & CCTV & I & 903 & $\begin{array}{l}\text { CCTV was used to facilitate } \\
\text { remote flushing of the overhead } \\
\text { tank. A significant amount of } \\
\text { mercury was removed, and the } \\
\text { bottom of the tank had some } \\
\text { sand/silt material with droplets of } \\
\text { mercury remaining. }\end{array}$ \\
\hline $\mathrm{H}$ & EVAP-25 & $\begin{array}{r}\text { Overhead } \\
\text { Tank \#2 }\end{array}$ & $01 / 19 / 03$ & CCTV & I & 903 & $\begin{array}{l}\text { CCTV was used to facilitate } \\
\text { remote flushing of the overhead } \\
\text { tank. The tank was cleaned with } \\
\text { only minor mercury droplets } \\
\text { visible with sand/silt on the } \\
\text { bottom. }\end{array}$ \\
\hline
\end{tabular}

Inspection was made to document conditions in cell after an increase in the sump was detected. No unusual conditions were observed.

Inspection was made to observe for leakage at the U1 nozzle. No liquid was observed; however, stains were observed on the side of the evaporator, on the nozzle plate and on the floor beneath the nozzle.

Inspection documented conditions of the evaporator cell, pot exterior, service piping, cell covers and jumpers. No unusual conditions were observed. Stains observed on the $\mathrm{U}-1$ nozzle plate have increased slightly, indicating leakage on the lift jumper to Tank 37 . The stains were first observed on $4 / 18 / 03$.

CCTV was used to leak check the S6 flange during evaporator operation. No leaks were observed. 


\begin{tabular}{|c|c|c|c|c|c|c|c|}
\hline \multirow{2}{*}{$\frac{\text { AREA }}{\mathrm{H}}$} & \multirow{2}{*}{$\begin{array}{c}\begin{array}{c}\text { TANK OR } \\
\text { ANCILLARY }\end{array} \\
\text { EVAP-25 }\end{array}$} & $\frac{\text { ACCESS OPENING }}{(A \text { OR I) }}$ & \multirow{2}{*}{$\frac{\text { DATE }}{02 / 08 / 03}$} & \multicolumn{3}{|c|}{$\frac{\text { INSPECTION METHOD }}{\text { IDENTIFICATION NUMBER }}$} & \multirow[b]{2}{*}{$\begin{array}{l}\text { REMARKS } \\
\text { Inspection of the evaporator pot } \\
\text { showed all areas to be free of } \\
\text { deposits. A thin scale was } \\
\text { observed on the tube bundle and } \\
\text { in some areas of the pot near the } \\
\text { operating level. Thirteen warming } \\
\text { coil loops were visible. No } \\
\text { significant abnormalities were } \\
\text { noted, and all observations were } \\
\text { consistent with normal evaporator } \\
\text { operations. }\end{array}$} \\
\hline & & S-06 (I) & & CCTV & I & 903 & \\
\hline
\end{tabular}

Inspection documented conditions of the pot. No solids were observed on the exposed surfaces of the tube bundle, the vessel walls, observable service piping, or warming coil. The liquid remaining in the vessel was clear, and very little solids, if any, were present at the bottom of the cone. No unusual conditions were observed, and observations were consistent with normal evaporator operation.

Inspection revealed several inches of water in the cell.

CCTV was used to deploy a conductivity probe in the drain cell. The conductivity probe was observed extending beyond the end of the stand pipe. 


\begin{tabular}{|c|c|c|c|c|c|c|c|}
\hline \multirow{2}{*}{$\frac{\text { AREA }}{\mathrm{H}}$} & \multirow{2}{*}{$\begin{array}{c}\begin{array}{c}\text { TANK OR } \\
\text { ANCILLARY }\end{array} \\
\begin{array}{c}\text { LDB Drain } \\
\text { Cell }\end{array}\end{array}$} & \multirow[t]{2}{*}{$\frac{\text { ACCESS OPENING }}{(A \text { OR I) }}$} & \multirow{2}{*}{$\frac{\text { DATE }}{01 / 13 / 03}$} & \multicolumn{3}{|c|}{$\begin{array}{l}\text { INSPECTION METHOD } \\
\text { IDENTIFICATION NUMBER }\end{array}$} & \multirow{2}{*}{$\begin{array}{l}\text { REMARKS } \\
\text { The conductivity probe was } \\
\text { deployed at the setpoint. } \\
\text { Abandoned hoses were observed } \\
\text { on the cell floor and in the sump. }\end{array}$} \\
\hline & & & & CCTV & I & 908 & \\
\hline $\mathrm{F}$ & PP-03 & LDB-01 & $05 / 02 / 03$ & CCTV & I & 908 & $\begin{array}{l}\text { The conductivity probe was } \\
\text { properly deployed per setpoint } \\
\text { document J-JX-G-0001. }\end{array}$ \\
\hline $\mathrm{F}$ & PP-03 & LDB-14 & $05 / 02 / 03$ & CCTV & I & 908 & $\begin{array}{l}\text { The conductivity probe was } \\
\text { properly deployed per setpoint } \\
\text { document J-JX-G-0001. }\end{array}$ \\
\hline $\mathrm{H}$ & PP-04 & SE & 08/06/03 & CCTV & I & 922 & $\begin{array}{l}\text { Inspection documented the } \\
\text { conditions in the pump pit. } \\
\text { Abandoned jumpers (rigid and } \\
\text { flex), hoses, tubing, plastic } \\
\text { sheeting and other extraneous } \\
\text { items were observed in the pump } \\
\text { pit. Liquid was observed on the } \\
\text { floor approximately } 12-18 \text { inches } \\
\text { deep. The passive vent was } \\
\text { unobstructed. }\end{array}$ \\
\hline
\end{tabular}

CCTV was used to monitor removal of liquid from pump pit.

The conductivity probe was properly deployed per setpoint document J-JX-G-0001.

01/08/03

CCTV / 908

Inspection revealed mud and rust in the standpipe.

The conductivity probe was properly deployed per setpoint document J-JX-G-0001.

The conductivity probe was properly deployed per setpoint document J-JX-G-0001.

CCTV was used to facilitate remote removal of jumper $8 \mathrm{~A}(\mathrm{HPP} 5) 1 \mathrm{~A}-2 \mathrm{~A}$ and installation of dummy connector heads. The passive vent was unobstructed.

The conductivity probe was properly deployed per setpoint document J-JX-G-0001.

The conductivity probe was properly deployed per setpoint document J-JX-G-0001. 


\begin{tabular}{|c|c|c|c|c|c|c|c|}
\hline \multirow{2}{*}{$\frac{\text { AREA }}{\mathrm{H}}$} & \multirow{2}{*}{$\begin{array}{c}\begin{array}{c}\text { TANK OR } \\
\text { ANCILLARY }\end{array} \\
\text { PP-06 }\end{array}$} & \multirow{2}{*}{$\begin{array}{c}\frac{\text { ACCESS OPENING }}{\text { (A OR I) }} \\
\text { LDB-07 }\end{array}$} & \multirow{2}{*}{$\frac{\text { DATE }}{05 / 01 / 03}$} & \multicolumn{3}{|c|}{$\frac{\text { INSPECTION METHOD }}{\text { IDENTIFICATION NUMBER }}$} & \multirow{2}{*}{$\begin{array}{l}\qquad \text { REMARKS } \\
\qquad \begin{array}{l}\text { The conductivity probe was } \\
\text { properly deployed per setpoint } \\
\text { document J-JX-G-0001. }\end{array}\end{array}$} \\
\hline & & & & CCTV & I & 908 & \\
\hline $\mathrm{H}$ & PP-06 & LDB-07 & $10 / 27 / 03$ & CCTV & I & 964 & $\begin{array}{l}\text { The conductivity probe was } \\
\text { properly deployed per setpoint } \\
\text { document J-JX-G-0001. }\end{array}$ \\
\hline $\mathrm{H}$ & PT-06 & $05 \quad$ (I) & 06/03/03 & CCTV & I & 976 & $\begin{array}{l}\text { Inspection documented conditions } \\
\text { in the pump tank. No solids or } \\
\text { deposits were observed. Air was } \\
\text { observed discharging through the } \\
\text { liquid at the dip tubes. The } \\
\text { passive vent was reinstalled, and } \\
\text { no obstructions were observed. }\end{array}$ \\
\hline $\mathrm{H}$ & PT-07 & $08 \quad(\mathrm{I})$ & $05 / 15 / 03$ & CCTV & I & 950 & $\begin{array}{l}\text { Inspection documented conditions } \\
\text { in the pump tank. Plastic was } \\
\text { observed hanging from the } \\
\text { pumps, floating on the surface and } \\
\text { on the baffles. No other unusual } \\
\text { conditions were observed. }\end{array}$ \\
\hline
\end{tabular}

Inspection documented the interior of the pump tank. Inspection revealed a piece of plastic hanging from the transfer pump and a few small pieces of plastic floating on the surface. No other unusual conditions were observed. During removal of camera some material was observed around nozzle 23 on the transfer pump.

Inspection documented conditions in the pump tank. No unusual conditions were observed. Solids had settled on the bottom of the tank.

CCTV was used to document installation of a submersible pump/grinder and to monitor operations. Material that had settled on the bottom of the tank was pulled through the grinder and suspended in the liquid preventing any viewing beneath the liquid. No unusual conditions were observed. 


\section{TANK OR \\ AREA

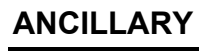

$\mathrm{H}$

PT-07

PT-08

$\mathrm{H}$

$\mathrm{H}$

PT-10

$\mathrm{H}$

WLE-02

$\mathrm{H}$

WLE-02

$\mathrm{F}$

WLE-05

\section{ACCESS OPENING (A OR I)}

DATE

18 (I)

$07 / 31 / 03$

04/08/03

CCTV

949

IDENTIFICATION NUMBER

CCTV / $\quad 997$

04/07/03

CCTV

947

$11 / 03 / 03$

CCTV

955

$11 / 22 / 03$

CCTV

955

$11 / 22 / 03$
CCTV /
REMARKS

CCTV was used to monitor removal of pump/grinder and document tank conditions. The liquid was dark and viewing through liquid was not possible. No unusual conditions were observed.

CCTV was used to facilitate the installation of the passive vent. The passive vent connector was installed, and no obstructions were observed.

Inspection was performed to document any formations of solids in the pump tank. No deposits were observed on the agitator, tank walls, pump or service piping. A small amount of solids had settled and covered the bottom of the tank.

Inspection documented the conditions of the encasement walls, cell floor, service piping and supports. Moisture was observed on the bottom of the cell covers and piping. Stains observed on the encasement walls are due to the inleakage of water.

Inspection documented the conditions of the encasement walls, cell floor, service piping and supports. No unusual conditions were observed. Condensation was observed on all exposed surfaces.

Inspection documented the conditions of the encasement walls, cell floor, service piping and supports. The encasement walls and floor are normal. Water intrusion was observed. No other unusual conditions were observed. 


\begin{tabular}{|c|c|c|c|c|}
\hline AREA & $\begin{array}{l}\text { TANK OR } \\
\text { ANCILLARY }\end{array}$ & $\frac{\text { ACCESS OPENING }}{(A \text { OR I) }}$ & DATE & $\frac{\text { INSPECTION METHOD }}{\text { IDENTIFICATION NUMBER }}$ \\
\hline $\mathrm{H}$ & WLE-05 & & $11 / 03 / 03$ & CCTV \\
\hline
\end{tabular}

\section{REMARKS}

Inspection documented the conditions of the encasement walls, cell floor, service piping and supports. Moisture was observed on the bottom of the cell covers and piping. The mastic material sagging and stains observed on the encasement walls are due to the inleakage of water. Mud was observed on the floor of the encasement.

Inspection documented the conditions of the encasement walls, cell floor, service piping and supports. Moisture was observed on the bottom of the cell covers and piping. The mastic material between the cell covers was observed sagging into the encasement, and stains on the encasement walls are due to the inleakage of water. Mud was observed on the floor of the encasement.

Inspection documented the conditions of the encasement walls, cell floor, service piping and supports. Moisture was observed on the bottom of the cell covers and piping. Stains observed on the encasement walls are due to the inleakage of water. Mud and extraneous items were observed on the floor of the encasement. 